$7{ }^{1}$ Toulouse Biotechnology Institute (TBI), UMR INSA-CNRS 5504 \& INRA 792; F-31077 Toulouse,

8 France,

92 Lallemand SAS, 19, rue des briquetiers, 31702 Blagnac, France growth.

\section{Physiological function of Flo11p domains and the particular role of amyloid core sequences}

$$
\text { of this adhesin in Saccharomyces cerevisiae }
$$

Clara Bouyx ${ }^{1}$, Marion Schiavone ${ }^{1,2}$, Marie-Ange Teste ${ }^{1}$, Etienne Dague ${ }^{3}$, Nathalie Sieczkowski ${ }^{2}$,

\section{${ }^{3}$ CNRS, LAAS F31400 Toulouse, France}

Email address of the authors:

\section{Clara Bouyx : $\quad$ bouyx@insa-toulouse.fr}

Marion Schiavone: $\quad$ schiavon@insa-toulouse.fr

Marie-Ange Teste : teste@insa-toulouse.fr

Etienne Dague: dague@laas.fr

Nathalie Sieczkowski : nsieczkowski@lallemand.com

Anne julien : ajulien@lallemand.com

Jean M François : $\quad$ fran jm@insa-toulouse.fr

*corresponding author

Short title: Functional analysis of Flo11 adhesin in yeast 


\section{ABSTRACT}

Flocculins are a family of glycosylated proteins that provide yeast cells with several properties such as biofilm formation, flocculation, invasive growth or formation of velum. These proteins are similarly organised with a $\mathrm{N}$-terminal (adhesion) domain, a stalk-like central B-domain with several repeats and a C-terminal sequence carrying a cell wall anchor site. They also contain amyloid $\beta$-aggregationprone sequences whose functional role is still unclear. In this work, we show that Flo11p differs from other flocculins by the presence of unique amyloid-forming sequences, whose the number is critical in the formation of adhesion nanodomains under a physical shear force. Using a genome editing approach to identify the function of domains in Flo11p phenotypes, we show that the formation of cellular aggregates whose density increases with the number of amyloid sequences cannot be attributed to a specific domain of Flo11p. The same is true for plastic adhesion and surface hydrophobicity the intensity of which depends mainly on the abundance of Flo11p on the cell surface. In contrast, the $\mathrm{N}$ and $\mathrm{C}$ domains of Flo11p are essential for invasive growth in agar, whereas a reduction in the number of repeats of the $B$ domain weakens this phenotype. However, expression of FLO11 alone is not sufficient to trigger this invasion phenotype. Finally, we show that this flocculin contributes to the integrity of the cell wall. 


\section{INTRODUCTION}

The yeast cell wall is a highly dynamic structure that is not only an armour separating cell from its surrounding but that is endowed of surface properties including adherence to inert material leading to biofilm formation, cell-cell adhesion that can yield to flocculation, hydrophobicity which may result in buoyant biofilms. These properties are mediated by a variety of surface proteins called adhesin or flocculin (Orlean, 2012, Lipke et al., 2018). In the pathogenic Candida albicans, it has been reported that adhesin encoded by $A L S 5$ can be organised into clusters of hundreds of proteins at the cell surface leading to highly adhesion nanodomains. This 3D-organisation is triggered by an external physical shear force, such as the extension force of single molecules stretching in AFM, which can propagate across the entire cell surface at a speed of about $20 \mathrm{~nm} \cdot \mathrm{min}^{-1}$ (Alsteens et al., 2010). Further works by these authors demonstrated that this clustering is mediated by amyloid-core $\beta$ aggregation sequences (IVIVATT) present in Als5 protein (Garcia et al., 2011). In addition, Als5p has an unusual high content of $\beta$-branched aliphatic Ile, Val, and Thr that can form $\beta$-aggregates structures as predicted by the $\beta$-aggregate predictor TANGO (http://tango.crg.es/), which contributes to the cis-interactions of these proteins on the cell surface. These amyloid-like sequences and the concurrent force-induced formation of adhesion nanodomains have been recognized for other $A L S$ encoding adhesins in Candida albicans (Otoo et al., 2008, Lipke et al., 2018). Altogether, the formation of nanodomains is an emergent property of the adhesin primary sequence, and it has been argued that the force-induced formation of these nanodomains is to strengthen cell-cell adhesion leading to the formation of robust biofilms, which promotes fungal infection (Lipke et al., 2018).

In a previous work aiming to investigate the impact of autolysis process on the nanomechanical properties of the cell wall of different Saccharomyces cerevisiae strains, we identified by Atomic Force Microscopy (AFM) the presence of abundant patches with a mean diameter of $140 \mathrm{~nm}$ at the surface of an industrial wine yeast strain that resembled adhesion nanodomains formed by clustering of Als 5 adhesin on the surface of $C$. albicans (Schiavone et al., 2015). Moreover, we found that these patches resulted from the aggregation of highly mannosylated 
proteins since they were responsive to the binding of concanavalin A functionalized AFM tips (ConA tip), showing rupture distances that spread out over a range of 50 to $500 \mathrm{~nm}$. We thus raised the hypothesis that the existence of these nanosized patches at the cell surface of this industrial yeast strain could be due to the aggregation of flocculins encoded by FLO genes family. The yeast Saccharomyces cerevisiae contains at least 5 genes encoding functional flocculins (FLO1, FLO5, FLO9, FLO10 and FLO11) that share a similar architecture comprising a N-terminal sequence (A-domain), a stalk-like, repetitive and highly glycosylated B-domain and a C-terminal sequence (C-domain) that carries a glycosylphosphatidylinositol (GPI) anchoring site for covalent attachment of the protein to $\beta$ (1,6)-glucans of the inner cell wall network (Dranginis et al., 2007, Orlean, 2012). These domains and pattern of repeats are nicely highlighted in the hydrophobic-cluster analysis (HCA) drawing shown in Figure 1. In addition, like C. albicans adhesins, the Saccharomyces cerevisiae flocculins contain $\beta$ aggregation sequences of 5 to 7 amino acids length that are rich in Ile, Val and Thr. The distribution of these repeats over the flocculins sequence enables to distinguish the group of Flo1p, Flo5p and Flo9p from that of Flo10p and Flo11p (Figure 1). In the former group, the $\beta$-aggregation-positive TANGO sequences are mainly characterized by the " $\mathrm{T}(\mathrm{V} / \mathrm{I}) \mathrm{IVI}$ " motif that is widely distributed over the B-domain (see details in Table S1). Interestingly, it was shown that a TDETVIVIRTP peptide containing the "TVIVI' motif forms amyloid fibers in vitro (Ramsook et al., 2010). On the other hand, $\beta$-aggregation prone sequences are far less abundant in Flo10p and Flo11p and are located in the Cdomain (Figure 1 and details in Table S1). It was furthermore reported that a 1331-residue soluble Flo11p can assemble into amyloid fibers in vitro most likely due to the presence of the amyloid- $\beta$ aggregation prone sequences "VVSTTV" and "VTTAVTT" at the C-terminus of Flo11p (Ramsook et al., 2010).

One of the purpose of this work was therefore to study the origin and the nature of the nanoscale patches formed on the cell surface of this industrial wine yeast strain, since such abundant nanostructures have never been physically observed before in Saccharomyces species, despite evidence of clustering of Flo1p or Flo11p on the cell surface to account for potentiation of cell-cell 
94

aggregation under hydrodynamic shear forces and its antagonism by anti-amyloid dyes (Ramsook et al., 2010, Chan \& Lipke, 2014, Chan et al., 2016). We showed in this report that these patches are adhesion nanodomains resulting from the force-induced clustering of Flo11p. We further found that the formation of these nanodomains was due to the presence of a unique sequence of 230 amino acid residues at the C-terminus of this protein, which may correspond to a sequence of 110 amino acids present in all Flo11p that is repeated two times. We then took the opportunity of this original Flo11p to investigate in greater details the importance of Flo11p domains in the various phenotypes including the formation of nanodomains that are elicited by this flocculin. This second objective was carried out by genome editing of FLO11 using the CRISPR-Cas9 tool leading to the expression at the FLO11 locus of Flo11p variants lacking either domain A, domain C, amyloid forming sequences, or lacking part of tandem repeats in the B-domain. Overall, this sequence-function analysis underscored that cell-cell aggregation critically depended on amyloid forming sequences, whose number potentiates this interaction leading eventually to the formation of adhesion nanodomains. However, these amyloid $\beta$-aggregation sequences have barely any effect on adherence, surface hydrophobicity and agar invasion, these later being mainly dependent on the $\mathrm{N}$ and the C-terminus of the Flo11p. Our results also indicate that Flo11p is necessary but not sufficient for the invasive growth phenotype and that high copy number of intragenic repeats in FLO11 as well as high expression of this gene are not sufficient arguments to explain the flocculation and velum formation phenotype dependent on this protein. 


\section{Biophysical characterisation of the nanostructures at the cell surface of L69 strain}

the contact of an AFM bare tip, their persistence in autolyzed cells (Schiavone et al., 2015) while they using quantitative imaging $\left(\mathrm{QI}^{\mathrm{TM}}\right)$ mode that enables both to image at high resolution and to quantify adhesive properties of the cell surface. Figure 2 reports such a height and adhesion images of a single cell from 669 strain trapped in the PDMS microchamber. By zooming in a small area of the embedded cell (the white square in Fig.2A), one can clearly see that the surface of the L69 strain is rough or even dotted. This apparent roughness can be accounted for proteins that cluster together on the cell surface. From the height image at high resolution (Fig.2B), we estimated that these clusters have an average diameter of $100 \mathrm{~nm}$ with a height over the cell surface in the range of $15-20 \mathrm{~nm}$ (Fig.2C). We then collected a total of 4096 force-distance curves from three independent cells to quantify the nanomechanical properties of the cell surface, namely adhesion forces and stiffness. Adhesion forces correspond to the retraction of the AFM tip from the surface, whereas the stiffness is the slope of the linear portion of the force versus indentation curve (see Fig.2E). These two physical parameters were reported as a function of the frequency of the interaction of the tip on the surface. Data of this analysis reported in Fig $\mathbf{2 F}$ and $\mathbf{2} \mathbf{H}$ showed a bimodal distribution of adhesion forces and stiffness, indicating the existence of two types of interactions. The first distribution that exhibited a mean adhesion force value of $589 \mathrm{pN}$ and mean stiffness of $5.7 \mathrm{nN} / \mu \mathrm{m}$ likely corresponded to hydrophobic interactions (Dague et al., 2007). The second distribution was characterized by weaker adhesion forces (mean value of $132 \mathrm{pN}$ ) and higher stiffness $(7.9 \mathrm{nN} / \mu \mathrm{m})$, which could be attributed to cell 
observation of two different biophysical properties of these nanodomains on the surface of the L69 yeast cells raises the question of whether they can be of the same or of different biological origins.

The formation of adhesion nanodomains can be inhibited by amyloid perturbants and is suppressed by deletion of FLO11.

The model of Lipke et al. (Lipke et al., 2012, Lipke et al., 2018) argues that the formation of adhesion nanodomains requires amyloid- $\beta$-aggregation prone sequences in the amino acids sequence of the involved proteins. The most straightforward experiment to verify this requirement was to challenge the formation of the nanodomains in the presence of amyloid perturbants such as anti-amyloid peptide (Alsteens et al., 2010, Lipke et al., 2012) or amyloidophilic dye thioflavine $S$ (Ramsook et al., 2010, Chan et al., 2016) that are reported to cancel the in vivo formation of nanodomains. Cells from L69 strain were thus treated with the amyloid disrupting peptide "VASTTVT", which is the mutated motif of the native 'VVSTTV' of Flo11p from BY4741 strain (Ramsook et al., 2010). As shown in Figure 3A, a 90-min incubation with this anti-amyloid peptide resulted in a complete disappearance of the highly adhesive nanodomains. There still remained some spots of weak adhesion with values ranging from 100 to $250 \mathrm{pN}$ that may likely correspond to low adhesive nanodomains as reported in Fig. 2 and which can be ascribed to isolated cell surface proteins getting unfolded upon AFM tip retraction. In contrast, the two types of nanodomains were completely abolished upon treatment with $10 \mu \mathrm{M}$ thioflavine $\mathrm{S}$ for $30 \mathrm{~min}$ (Fig 3B). Altogether, these data support the notion that the patches on the cell surface of L69 strain are resolved into two types of nanodomains, a low adhesive, apparently amyloid-independent, and a highly adhesive, amyloiddependent nanodomains that are triggered by application of the mechanical force such as extension force by the retraction of AFM tip.

Based on our previous work showing that FLO11 was the most highly expressed gene of the FLO family in strain L69 (Schiavone et al., 2015), and with the finding that the Flo11 protein of S288c harbours two typical amyloid core sequences "VVSTTV" and "VTTAVT" (Ramsook et al., 2010) near the C-terminus, we investigated the effect of deleting FLO11 in L69 strain using the CRISPR-Cas9 
toolbox (Mans et al., 2015). Since L69 strain is diploid (data not shown), we verified by RT-qPCR that L69flo11 did not express any FLO11 transcript, proving that both copies of this gene was deleted (see Figure S2A in supplementary data). We then carried out AFM analysis in a $\mathrm{QI}^{\mathrm{TM}}$ mode and found that $\mathrm{L69}$ defective in FLO11 had lost the ability to form adhesion nanodomains on its surface (Figure 3C). This result could be an explanation of the lack of nanodomains formation in the laboratory BY4741 strain, which derives from S288c (see Figure S1) since FLO11 is not expressed in this strain due to a nonsense mutation in FLO8 that encodes a major transcriptional activator of this gene (Liu et al., 1996, Kobayashi et al., 1999). To verify this explanation, we retrieved FLO11 gene sequence of S288c as well as that of FLO11 from L69 strain whose genome was recently sequenced (Lallemand Inc unpublished data). Interestingly, we found that the FLO11 gene (provisional GenBank accession number Banklt2416107 Seq1MW448340) of this industrial strain was $1.06 \mathrm{kbp}$ longer than that of BY4741 (ie 5.16 vs $4.10 \mathrm{kbp}$ ). Both genes were cloned into the high copy pYES2.1 plasmid under the control of GAL1 promoter. The corresponding plasmids were used to transform BYflo11 strain (BY4741 deleted for FLO11 by replacement with the KanMX4 cassette) and the yeast transformants were cultivated in YNGal medium that warranted its huge overexpression (see data in Figure S2B showing that transcript levels of $F L O 11^{B Y}$ and $F L O 11^{L 69}$ were respectively 2000 and 24000 fold higher than in the non-transformed BYflo11 1 cultivated on a galactose medium). Results undoubtedly showed that only overexpression of FLO11 arising from L69 strain (FLO11 ${ }^{L 69}$ gene) conferred the capability of the laboratory strain to produce nanodomains (Figure 3C). From these results, it can be concluded that the lack of nanodomains formation in BY4741 is not solely due the absence of FLO11 but that the formation of these unique nanostructures requires additional features that are present in the amino acid sequence of the Flo11 protein from L69 strain.

Comparative sequence analysis of Flo11p from various yeast strains and construction of Flo11p variants from L69 strain Using Clustal Omega, we aligned the amino acids sequence of Flo11p from L69 strain (provisional GenBank accession number Banklt2416107 Seq1MW448340) with that of different 
Saccharomyces cerevisiae strain, including the originally genome-sequenced strain S288c (Goffeau, 1998), $\Sigma 1278 b$ (Dowell et al., 2010), a strain widely studied for its remarkable properties of colony morphology, invasive and pseudohyphal growth (Reynolds \& Fink, 2001, Dowell et al., 2010, Voordeckers et al., 2015) and the flor yeast strain 133d reported to form buoyancy biofilms also termed velum (Fidalgo et al., 2006). We found that Flo11p $\mathrm{p}^{\mathrm{L} 69}$ is 355 and 512 amino acids longer than Flo11 $p^{\mathrm{BY}}$ and Flo11 $\mathrm{p}^{\Sigma}$, respectively, but only 92 amino acids longer than Flo11p from strain 133d (see Figure S3 in supplementary data). This alignment also revealed that Flo11p ${ }^{169}$ presented a sequence of about 230 amino acids long near the C-terminus that was totally absent in the others Flo11 proteins (see Figure S3, as highlighted by a red box). More remarkably, a BLAST analysis performed on all Saccharomyces cerevisiae strain sequenced to date revealed that this amino acids sequence was not present in Flo11p of these sequenced strains.

As already indicated in the introduction, the hydrophobicity cluster analysis tool (HCA, https://mobyle.rpbs.univ-paris-diderot.fr/cgi-bin/portal.py?form=HCA\#forms::HCA) is well adapted to compare proteins having repeated sequences and high percentage of hydrophobic amino acids and hence to make emphasis on their similarity and disparity (Lo \& Dranginis, 1996). As depicted in Figure 4 (see also Figure S4 to S7 in supplementary data for more visual details on HCA analysis of these Flo11 proteins), the Flo11 proteins of strains L69, BY4741, $11278 \mathrm{~b}$ and $133 \mathrm{~d}$ all have the same sequence architecture with an "A domain" at the $\mathrm{N}$-terminus, a central B domain containing several serine/threonine (TR)-rich tandem repeats and a C-terminus carrying the GPI anchor site for attachment to the cell wall network. However, there are major differences between the Flo11p of L69 strain and that from the other yeast strains. In particular, Flo11p ${ }^{69}$ contains more repeats (TRs) than the proteins from BY4741 and $\Sigma 1278 b$ (Figure 4), but slightly less (46 vs 49) than Flo11p from the Flor strain 133d. Interestingly, a search for the intragenic repeats in FLO11 using EMBOSS TANDEM software (https://www.bioinformatics.nl/cgi-bin/emboss/etandem) indicated that FLO11 from strain $133 \mathrm{~d}$ harbored a same repeat of $81 \mathrm{nt}$ that is repeated 49 times, whereas 4 different intragenic repeats with different nucleotide size were identified in the other FLO11 genes (see details 
on intragenic repeats in Table S2 in supplementary data). A second difference was in the presence in

Flo11p $p^{\text {L69 }}$ of two additional amyloid core sequences "VVSTTV", which possess $75.7 \%$-aggregation

potential (Table 1). A closer inspection of these amino acid residues may suggest that a sequence of

about 115 amino acids length has been duplicated giving rise to an additional 230 amino acid

in the formation of adhesion nanodomains. To answer this question, we constructed a variant of deletion of the repeat region RR1 which matches more than $90 \%$ the sequence in Flo $11 p^{\mathrm{BY}}$. The genome engineering of $F L O 11$ was verified by PCR to confirm that these modifications were in both copies of the gene. Moreover, expression of the genes encoding the different Flo $11 p^{\text {L69 }}$ variants was verified by determining transcript levels by quantitative reverse transcription (qRT) PCR on total RNA

level about two times lower than that of the wild type gene.

The Flo11p of strain L69 has additional amyloid $\beta$-aggregation sequences that are responsible for nanodomains formation. 
argued that the expression of these Flo11p ${ }^{\mathrm{L} 69}$ variants had no impact on the global surface topology of yeast cell (Fig. 5A, left panel). However, the adhesion images at high resolution clearly revealed that yeast cells expressing the Flo11 ${ }^{69}$ variant lacking RR2 region (Flo11- $\Delta$ RR2) was unable to produce nanodomains under the AFM tip, while the presence of nanodomains was still recorded on the surface of yeast cell that expressed a Flo11p ${ }^{\mathrm{L} 69}$ lacking either the $\mathrm{N}$ or the C-terminus, although the size and the morphology of these patches were different from those imaged on the surface of a L69 yeast cell expressing the wild type Flo11p (compare Fig. 5A with Fig 1A). In support of this observation, we found that nanodomains on the surface of yeast cells expressing the Flo11- $\Delta$ Nter variant had a unimodal distribution of adhesion force that peaked at a maximal value of $104 \mathrm{pN}$. Moreover, these nanodomains were less stiff than those of the L69 strain expressing the wild type Flo11p (see Figure $\mathbf{S 8}$ in supplementary data). On the other hand, the cell surface of L69flo11- $\Delta$ Cter cell was characterized by needle-shaped nanostructures whose height was 3 times greater than nanodomains formed on the surface of the L69 strain. These nanodomains displayed adhesion forces that were scattered from a few $\mathrm{pN}$ to $\max 200 \mathrm{pN}$ and stiffness that was roughly $30 \%$ higher than that of nanodomains from L69 strain (see Figure S9, in supplementary data). Finally, some tiny and disparate patches were noticeable on the surface of a L69flo11- $\Delta R R 1$ cell that expresses a Flo11p variant in which $40 \%$ of the tandem repeats in B-domain has been removed (deletion of RR1 region, see Figure 5A). Typical force-distance curves recorded on these needle-shape spots showed that the adhesion forces were very weak, in the range of $100 \mathrm{pN}$ (see Figure S10 in Supplementary data), suggesting that these spots may correspond to small clusters of either Flo11 protein variant or other isolated cell wall proteins getting unfolded upon retraction of AFM tip. Taken together, these results indicated that the RR2 sequence in the Flo11 protein of L69 strain is critically important for the formation of highly adhesive nanodomains under the AFM tip, and hence suggested that a threshold number of amyloid-forming sequence is required for the formation of adhesion nanodomains.

To support this assertion, we inserted the RR2 sequence of Flo11p ${ }^{\mathrm{L} 69}$ into the Flo11p of BY4741 and cloned this construct in pYES2.1 under the GAL1 promoter. As shown in Figure 5B, low and high 
adhesive nanodomains were observed at the cell surface of the laboratory strain BY4741 upon ectopic overexpression of the chimeric gene encoding the Flo11 ${ }^{\mathrm{BY}}-[\mathrm{RR} 2]^{\mathrm{Lg}}$ protein variant. The nanomechanical values of these nanodomains were however slightly different from those determined on the cell surface of the L69 strain, probably because the overall cell wall architecture of cells that overexpressed this chimeric gene is different from that of L69 cells. Nonetheless, these results confirmed the importance of the RR2 region in the force-induced formation of nanodomains, which supports the notion that a minimal threshold of amyloid core sequences within the Flo11 protein is needed to elicit this event.

\section{Cell-cell aggregation is enhanced by increasing the number of amyloid forming sequences}

A well-established function of yeast flocculins is to promote cell-cell aggregation which can lead eventually to flocculation (Verstrepen \& Klis, 2006), or to buoyant biofilm (also termed velum) that are formed by wild yeast termed 'flor yeasts' (Alexandre, 2013). It is considered that cell-cell aggregation proceeds by two consecutive and possibly interdependent actions, namely a cell-cell adhesion that was found to largely depend on the $\mathrm{N}$-terminal domain also termed the $\mathrm{A}$ (adhesion) domain (Kraushaar et al., 2015), followed by a potentiation of these interactions by a mechanism called 'catch bonds'. It was reported that this catch bonding is dependent on the presence of force sensitive amyloid sequences, which can lead to robust biofilms (Lipke et al., 2012, Lipke et al., 2018). Cell-cell aggregation can be easily monitored under an optical microscope by the appearance of clumps or aggregates of several cells. We quantified the intensity of this event taking into account that an aggregate must contain at least 5 cells. As shown in Figure 6A, both strains L69 and YSWT3a that derived from $\Sigma 1278 \mathrm{~b}$ nicely exhibited this clumping phenotype. Deletion of FLO11 in L69 strain almost completely abrogated the formation of aggregates whereas in the $\Sigma 1278 \mathrm{~b}$ background strain, the size of these aggregates were dramatically reduced but surprisingly, it remained small aggregates of less than 10 cells. This residual clumping is likely a specific feature from $\Sigma 1278 \mathrm{~b}$ background strain, which does not hamper the notion that Flo11p is critical in cell-cell interaction. This is further 
supported by the lack of aggregates in BY4741 as this strain does not express FLO11 due to nonsense mutation in FLO8 encoding its major transcriptional activator.

To investigate the role of amyloid core sequence in the cell-cell aggregation, we carried out these two following experiments. On the one hand, BYflo11 $\Delta$ strain that does not form aggregates was transformed with a high copy pYES2.1 plasmid carrying either its own FLO11 gene, FLO11 from L69 strain or a chimeric gene construct in which a DNA fragment corresponding to RR2 sequence of $F L O 11^{L 69}$ was inserted into FLO11 of BY4741 strain to yield $F L O 11^{\mathrm{BY}}-[\mathrm{RR} 2]^{\mathrm{LG}}$. Following growth on galactose, cell aggregates were observed upon ectopic overexpression of these FLO11 genes (Fig. 6A). However, this phenotype was much more pronounced in BYflo11 that overexpresses $\mathrm{FLO11}{ }^{\mathrm{LG}}$, reaching a percentage of cellular aggregates similar to that of strain L69. Moreover, expression of the chimeric construct $F L O 11^{B Y}-R R 2^{L 69}$ had a statistically stronger effect in aggregates formation than that of $\mathrm{FLO11}^{\mathrm{BY}}$ (Figure 6B, see also Figure S11 in supplementary data). Complementary to this experiment, the role of amyloid core sequences in cell-cell interaction could be assessed using amyloid perturbants. We found that aggregates formed in L69 strain or in BY $\Delta$ flo11 that overexpressed $F L O 11^{L 69}$ were largely disrupted upon incubation with the amyloid disruptor Thioflavine S, whereas this drug had almost no effect in L69flo11- $\Delta R R 2$ strain that expresses a Flo11p variant lacking all amyloid-forming sequences (Figure S11, in supplementary data). Very interestingly, cell aggregation in YSWT3 $\alpha$ strain was not altered by incubation with Thioflavin S, highlighting a strain background specificity for this phenotype that may be dependent on specific features in Flo11p sequence of this strain, with notably the unique presence of a 15 amino acids sequence in the A-domain that may confer stronger cell-cell adhesion property (Bruckner et al., 2020).

To further demonstrate the importance of amyloid core sequences in cell-cell interactions, we evaluated the effect of removing the RR2 region that bears all the 4 amyloid-forming sequence. As shown in Figure 6, ablation of this region in Flo11p resulted in a $75 \%$ reduction of the cell-cell interaction, and this reduction was statistically higher than that of the removal of the A-domain (Flo11- $\Delta$ Nter) (Figure 6). The contribution of the other domains of Flo11p to this phenotype was also 
evaluated. The percentage of cell aggregates dropped by approximately $50 \%$ upon deletion of the Cterminal, which could be accounted by the loss of 2 out of the 4 amyloid forming sequence. A $50 \%$ reduction of cell aggregates was also observed in the strain that expresses Flo11p lacking part of the B-domain (RR1 sequence). Altogether, these results indicated that the in vivo efficiency of cell-cell interaction requires the full Flo11 protein.

\section{Contribution of various domains of Flo11p to plastic adherence and surface hydrophobicity.}

Using mutant strains expressing variant proteins of Flo11 $p^{\text {L69 }}$, the contribution of each domain and of the amyloid core sequences on adherence and hydrophobicity properties dependent of Flo11p (Lo \& Dranginis, 1996, Lo \& Dranginis, 1998, Reynolds \& Fink, 2001, Dranginis et al., 2007) could be assessed. Adherence was assayed on polystyrene surface. We found that adherence to plastic was strongly affected by $\mathrm{pH}$ of the culture medium, but this $\mathrm{pH}$-dependency was no similar between yeast strains. While adherence to plastic of strain L69 and BY4741 was significantly reduced by raising $\mathrm{pH}$ from 5.0 to 8.0 , it however increased in the $\Sigma 1278$ derived haploid strain YSTW $\alpha$. Also this property involved other cell wall proteins since deletion of FLO11 significantly reduced but not annihilate adherence of cells to the polystyrene (Figure 7). Moreover, the moderate adherence of BY4741 cell to plastic at pH 5.0 must be due to cell wall proteins other than Flo11p, since this gene is not expressed in this strain due to a non-sense mutation in FLO8 encoding its transcriptional activator (Liu et al., 1996). However, the critical role of Flo11p is in adherence to inert surface was clearly illustrated in BY $\Delta$ flo11 strains that overexpressed FLO11 gene (Figure 7). However, it is worth noticing that cells overexpressing $F L O 11^{L 69}$ had higher adherence to plastic $(p$-value $<0.005)$ than those expressing $F L O 11^{B Y}$, whether the assay was carried out at $\mathrm{pH} 5.0$ or 8.0, although the intensity of this phenotype was lower at $\mathrm{pH}$ 8.0. This difference was probably due to the strong expression of $F L O 11^{169}$ rather than to additional presence of amyloid sequences in Flo11 $p^{69}$ since overexpression of the chimeric construct $F L O 11^{B Y}-R R 2^{L 69}$ in $\mathrm{BY} f l 011 \Delta$ cells gave rise to similar adherence as overexpression of $F L O 11^{B Y}$. Further analysis of the domains of Flo11p that are implied in adherence to polystyrene showed that the C-terminal domain contributed the most to this property at $\mathrm{pH} 5.0$, 
whereas at $\mathrm{pH} 8.0$, this contribution relied almost exclusively on the $\mathrm{N}$-terminal domain of this protein. This result was in part different from the data of Krausahaar et al. (Kraushaar et al., 2015) who showed that only the A-domain ( $\mathrm{N}$-ter) of Flo11p is required for cell adhesion to polystyrene.

Results on surface hydrophobicity, which was evaluated as the percentage of yeast cell partitioned in the octane phase, showed that each domain of Flo11p contributed to the intensity of this phenotype, with the $\mathrm{N}$-terminal carrying the higher contribution and the $\mathrm{C}$-terminal the lowest. However, this phenotype is mainly dependent on the amount of Flo11p exposed at the cell surface as shown by the significant increase in hydrophobicity of the cells that overexpressed FLO11, regardless the strain that originated this gene (Figure 8). Since surface hydrophobicity is critical in the formation of buoyant biofilm that is strictly dependent on Flo11p (Fidalgo et al., 2006), we also examined whether L69 strain, which is a wine yeast had this ability to form this air-liquid biofilm also termed velum (Alexandre, 2013). Contrary to expectation, neither L69 strains nor strain bearing any of the Flo11p variant as well as BY4741 were able to form velum, whereas this phenotype was nicely visualized in the flor strain A9 as described in ((Zara et al., 2005), Figure S12 in supplementary data). Taking into account that the number of repeats in the B domain is of paramount importance in eliciting velum formation (Fidalgo et al., 2006), the absence of this phenotype in strain L69 cannot be attributed to the lack of domain repeats, since there are almost as many in Flo11p of strain L69 as in the protein of strain $133 \mathrm{~d}$, nor to the expression of FLO11, since it is particularly strong in strain L69.

The Flo11p-dependent Invasive growth phenotype requires $\mathbf{N}$ and C-terminal of the protein, but its elicitation and intensity requests other factors that are defective in S288c background strain.

It is well established that FLO11 gene is required for pseudohyphal development in diploids and for invasive growth in haploids strains of S. cerevisiae (Gancedo, 2001), although the invasive phenotype can be provoked in diploid strains upon overexpression of this gene (Lo \& Dranginis, 1998). This phenotype is commonly assessed by agar invasion assay that consists in cultivating yeast cells on agar plates containing rich or synthetic sugar medium for several days and then examining those cells that remained sticky on the agar plates after extensive washing under a stream of water 
373 (Roberts \& Fink, 1994). Applying this assay to L69 strain, we found that this strain exhibited massive

374 invasion in agar on both rich (YPD) and synthetic (YNGal) sugar medium (Figure 9). The finding that invasiveness on agar occurred in this strain, which is diploid may be attributed to the relatively high expression of FLO11 measured in this strain (Schiavone et al., 2015). As expected, agar invasion was noticed for the haploid YSWT3 $\alpha$ strain, which is derived from $\Sigma 1278 b$ (Dowell et al., 2010), whereas 1996). Accordingly, invasion phenotype was lost upon deletion of FLO11 in L69 and YSTW3 $\alpha$ strains, which confirmed the critical function of this gene for this phenotype (Lo \& Dranginis, 1998). As already noticed by Guo et al. (Guo et al., 2000), invasion was less pronounced on agar plates made with a galactose medium (see Figure $\mathbf{S 1 2}$ in supplementary data). In addition we found that cells remaining the more sticky after washing are those at the periphery of the spot when the invasion experiment was carried out in a rich sugar medium, whereas cells at the heart of the spot showed the most invasiveness in a synthetic medium (Figure $\mathbf{8}$ and see also Figure $\mathbf{1 1}$ in supplementary data). More surprisingly, the diploid $\Sigma 1278 \mathrm{~b}$ and isogenic derivative haploid YSTW3 $\alpha$ were unable to elicit an invasion growth phenotype in a synthetic glucose medium, whereas this capacity was still very effective in L69 strain (see Figure S12 in supplementary data). vivo invasive growth phenotype. Results in Figure $\mathbf{9}$ show that the invasion in agar was completely lost in strains expressing a Flo11p that lacks either the $\mathrm{N}$ or the C-terminal domain. This phenotype was reduced in L69flo11- $\triangle R R 1$ strain cells, whereas the removal of the RR2 sequence (L69flo11$\Delta R R 2)$ had barely no effect on the capacity of cells to invade agar (Figure 9 and see also Figure S12). From these results, it can be concluded that RR2 region, which is needed for the formation of adhesive nanodomains, has no role in the invasion growth phenotype. More puzzling results were obtained with the laboratory strain BY4741 transformed with $F L O 11^{L 69}$ or $F L O 11^{B Y}$ carried on a $2 \mu$ plasmid under the GAL1 promoter (pYES2.1). Indeed, we found that this strain failed to invade agar 
Ura') (Figure 9B) under which these genes were shown to be exceedingly transcribed (see Figure S2).

400 On the other hand, the invasiveness of the haploid YSWT3 $\alpha$ deleted for 401 FLO11 ( flo11 $\Delta$ ) transformed with pYES2.1 plasmid carrying either $F L O 11^{B \gamma}$ or $F L O 11^{L 69}$ was clearly

402

403

discernable in the selective YNGal Ura'(Figure 8B), with $F L O 11^{L 69}$ being slightly more effective than $F L O 11^{B Y}$. Nonetheless, the invasive phenotype of $\Sigma f l o 11 \Delta$ that overexpressed $F L O 11^{B Y}$ or $F L O 11^{L 69}$ was weaker than that of this strain expressing its endogenous Flo11 protein.

\section{Flo11 protein and the cell wall integrity}

Flo11p is a highly mannosylated cell surface protein that is retained to the cell wall inner network of $\beta-1,6$ glucans through a GPI anchor (Klis et al., 2006). We found that the loss of function of this gene resulted in a higher sensitivity to drugs such as Calcofluor White (CFW), caffeine and Congo Red (CR) that are commonly used to assess integrity of the yeast cell wall (Levin, 2005). Furthermore, we found that the higher sensitivity to these cell wall drugs was mainly associated with the lack of the C-terminal region (from aa 1297 to 1721, see Fig. 3) of Flo11p although removal of RR1 or RR2 sequence of Flo11 ${ }^{\text {L9 }}$ protein resulted in mutant strains that were also slightly more sensitive than wild type (see Figure S13). The higher sensitivity of L69flo11-ACter strain could be explained by the lack of retention of the protein at the cell surface due to the removal of the GPI anchor. Under glycosylation of Flo11p could be invoked for the slight increase of sensitivity to cell wall drugs of strains expressing variants of $F / 011^{69} p$ that have been deleted for RR1 since the repeated sequences in this region are thought to be heavily glycosylated and cell surface glycosylation is important in the cell wall integrity (Strahl-Bolsinger et al., 1999, Free, 2013). 


\section{DISCUSSION}

In this work, we have demonstrated that the abundant and dense patches observed by AFM on the cell surface of the industrial wine yeast L69 strain (Schiavone et al., 2015) correspond to adhesion nanodomains, with similar physical characteristics as those formed on the cell surface of the pathogen C. albicans (Alsteens et al., 2010, Formosa et al., 2015). We moreover showed that the flocculin encoded by FLO11 is the sole protein involved in the formation of these nanodomains, and this event can be prevented upon treatment of the cells with anti-amyloid peptides or antiamyloidophilic dyes. Altogether, these data are in line with the model of Lipke et al. (Lipke et al., 2012, Lipke et al., 2018) arguing that adhesion nanodomains require wall-anchored proteins that must harbour in their protein sequence the following features: (i) amyloid- $\beta$-aggregation prone sequences of 5 to 7 amino acid residues and (ii) serine/threonine-rich 'T domain' enriched of $\beta$ branched aliphatic amino acids Ile, Val and Thr. While all flocculins encoded by FLO1, FLO5, FLO9 and FLO11 in yeast display these criteria, it was intriguing to know why such dense and abundant nanodomains had not been observed so far in Saccharomyces cerevisiae, even though physical FLOdependent- modification of cell surface upon hydrodynamic shear force have been recorded (Chan \& Lipke, 2014, Chan et al., 2016). The data reported in this work clearly showed that Flo11 protein from L69 strain differs from that of Saccharomyces strains sequenced so far, including the laboratory strains BY4741, $\Sigma 1278 \mathrm{~b}$ and the Flor strain 113d (Fidalgo et al., 2006), by having a unique 230 amino acids sequence termed RR2 near the C-terminus. Interestingly, this extra sequence is likely the result of a duplication of a region of about 115 amino acids located at the C-terminal of Flo11 flocculins, which is characterized by the presence of $\beta$-aggregation sequence "VVSTTV" followed by "ITTTFV". This duplication thus provides 2 additional amyloid-core sequences, which are clearly essential in the process of nanodomains formation in response to a tensile force, as demonstrated by the insertion of RR2 sequence into the Flo11p of BY4741 enabling this strain to form adhesion nanodomains with similar physical properties as those formed on the cell surface of L69 strain. 

importance of amyloid forming sequences in the cell surface properties conferred by this protein, and by extension on the role of $A, B$ and $C$ domains in the in vivo function of Flo11p in the yeast potentiated by increasing the number of amyloid-forming sequences in Flo11p. The finding that this phenotype was not abrogated when domain A was deleted could be at first glance in contradiction with the data of Kraushaar et al. (Kraushaar et al., 2015) who showed that this domain is essential for conferring cell-cell adhesion by facilitating homotypic interactions. However, the lack of A domain can be in part overcome by amyloid-forming sequences as these later could promote clustering of Flo11 molecules in cis, which enables cell-cell adhesion by trans-interaction as proposed by these same authors (Kraushaar et al., 2015, Bruckner et al., 2020). As a consequence, one can predict a complete loss of cell-cell aggregation would require ablation of both A-domain and RR2 in Flo11p. incorrect because not solely this sequence does not exist in Flo11p of L69 and BY4741 strains (see

469 Figure S3 in supplementary data), but, in addition, Bruckner et al (Bruckner et al., 2020) showed that this 15 amino acids sequence actually enhanced cell-cell adhesion. 

properties that Flo11p confers to yeast cells. Here, we showed that these two phenotypes are mainly dependent on the abundance of Flo11p at the surface of the yeast cell, whatever their strain origin. Nevertheless, the fact that the intensity of plastic adhesion was dependent on $\mathrm{pH}$ was not unexpected, except that it was surprising to find that this dependence was not solely attributed to the N-terminal that carries A-domain of Flo11p, as reported by (Kraushaar et al., 2015). In our hands, we found no role of A-domain in adherence to plastic at $\mathrm{pH} 5.0$, whereas this domain has a major role at $\mathrm{pH}$ 8.0. How can we reconcile these apparent discrepancies? A likely explanation would be to consider that the contribution of Flo11p to plastic adherence at pH 5.0 is relatively weak, whereas this contribution is proportionally higher at $\mathrm{pH}$ 8.0. Arguments in favour of this suggestion are found in the fact that adherence of BY4741 at pH 5.0 is independent to FLO11, that the drop in adherence upon loss of FLO11 is more pronounced at pH8.0, and that adherence of YSWT $\alpha$ strain at $\mathrm{pH} 8.0$ is much higher than at pH 5.0. On the other hand, Kraushaar et al (Kraushaar et al., 2015) could not make this observation because they were working with a strain in which FLO11 gene was overexpressed, which clearly exacerbated adherence to plastic, and in consequence have masked effect of other cell wall proteins in this property. At variance to the role of $\mathrm{N}$-ter in the adherence phenotype, the hydrophobicity property of Flo11p is dependent on a cooperation of various domains of this protein, although the $\mathrm{N}$-terminus and the central $\mathrm{B}$-domain contributed most to this property. due to the presence of several tryptophan residues (Lo \& Dranginis, 1998, Mortensen et al., 2007, in the B-domain of Flo11p (Fidalgo et al., 2006, Zara et al., 2009). However, this wine strain did not elicit velum formation under a flor medium condition in spite of the fact that the number of repeats in Flo11p of L69 strain is similar to that of Flo11p from the flor yeast strain 133d (46 vs 49) and higher 495 than Flo11p of $\mathrm{S} 288 \mathrm{c}$ or $\Sigma 1278 \mathrm{~b}$ ( 39 and 23 , respectively). Two possible, and nonexclusive, 
other yeast strain to form velum. This can be due to the nature of the repeats as the FLO11 gene of strain $133 \mathrm{~d}$ presents a single type of sequence repeat of $81 \mathrm{nt}$ whereas all other FLO11 harbour at least 4 different sequence repeats. Alternatively or complementary, Flo11p of strain $133 \mathrm{~d}$ is more glycosylated than that of Flo11p of strain 69 in spite of the protein have similar number of repeats.

A dominant phenotype brought about by expression of FLO11 is the ability of haploid yeast cells to undergo invasive growth in agar or pseudohyphal growth for a diploid cell (Gancedo, 2001). In this work, we found that L69 exhibited a very intense invasive growth phenotype, which was at first glance surprising since L69 strain is diploid. This phenotype is commonly not expressed in diploid cells unless FLO11 is highly expressed (Lo \& Dranginis, 1998), supporting our previous data that this gene is highly expressed in this strain (Schiavone et al., 2015). We moreover showed that the N-ter and the C-ter of Flo11p are indispensable to elicit agar invasion phenotype. While it was already reported that invasion in agar was abrogated upon deletion of the $\mathrm{N}$-terminus that carries the Adomain of Flo11p (Kraushaar et al., 2015), the importance of C-terminus in this phenotype had never been reported. This result could be explained by the inability of the cell to remain trapped in the agar due to the fact that Flo11p, in the absence of its C-terminus, can no longer remain attached to the cell wall network. On the other hand, the invasive growth in agar was strongly diminished upon deletion of repeat sequences in the B-domain. This result can be explained by either one of these two possibilities or a combination of both. Either, it is due to a reduction of glycosylation of Flo11p as a defect in this process was found to reduce invasive growth in agar (Meem \& Cullen, 2012). Alternatively, the removal of tandem repeat in B-domain (ie RR1 sequence) may prevent Flo11p from reaching the cell surface and therefore reduce the capacity of the cells to remain trapped in the agar. Finally, this study brought to light new data on the process of invasive growth. On the one hand, we found that invasion in agar of L69 strain was equally intense under any kind of growth media, whereas this phenotype in the well-established $\Sigma 1278 \mathrm{~b}$ background strain was weak in a galactose agar medium and absent in a glucose agar synthetic medium. In addition, the efficient agar invasion phenotype in $L 69$ strain could not be solely attributed to FLO11 gene because the overexpression of 
523 this gene in YSTW3a flo11 $\Delta$ (derivative of $\Sigma 1278 b$ ) did not provide to this strain a higher capacity to

524 invade agar than that of the isogenic YSTW3 $\alpha$ expressing its endogenous gene. On the other hand,

525 the ectopic overexpression of FLO11 in BY4741 did not rescue the inability of this haploid strain to

526 elicit invasive growth phenotype. Altogether, these data indicated that the Flo11p-dependent

527 invasive phenotype involves additional genetic factors that are absent in BY4741 and that are more

528 expressed in L69 strain, accounting for the higher potency of this strain to invade agar.

529

530 


\section{MATERIALS AND METHODS}

532

533

534

535

\section{Strains and growth conditions}

The Saccharomyces cerevisiae strains used in this work are listed in Table S4. Unless otherwise stated, strains were cultivated in rich YPD medium at $30^{\circ} \mathrm{C}(1 \% \mathrm{w} / \mathrm{v}$ yeast extract, $1 \% \mathrm{w} / \mathrm{v}$ bactopeptone and $2 \% \mathrm{w} / \mathrm{v}$ glucose), When using synthetic YNGlu or YNGal media $\left(0.17 \% \mathrm{w} / \mathrm{v}\right.$ yeast nitrogen base, $0.5 \% \mathrm{w} / \mathrm{v}\left(\mathrm{NH}_{4}\right)_{2} \mathrm{SO}_{4}$, with $2 \% \mathrm{w} / \mathrm{v}$ glucose or $2 \% \mathrm{w} / \mathrm{v}$ galactose), they were supplemented with appropriate amino acids at $0.1 \%$ (w:v). YNB Acetamide medium $\left(0.17 \% \mathrm{w} / \mathrm{v}\right.$ yeast nitrogen base, $0.66 \% \mathrm{w} / \mathrm{v} \mathrm{K}_{2} \mathrm{SO}_{4}, 0.06 \% \mathrm{w} / \mathrm{v}$ acetamide and $2 \%$ w/v glucose) was used for the selection and propagation of L69 mutants transformed with plasmid bearing amdSYM cassette (Solis-Escalante et al., 2013) used for CRISPR-Cas9 deletion (Ryan et al., 2016). YNB Fluoroacetamide medium $\left(0.17 \% \mathrm{w} / \mathrm{v}\right.$ of yeast nitrogen base, $0.5 \% \mathrm{w} / \mathrm{v}$ of $\left(\mathrm{NH}_{4}\right) 2 \mathrm{SO}_{4}, 0.23 \% \mathrm{w} / \mathrm{v}$ fluoroacetamide and $2 \% \mathrm{w} / \mathrm{v}$ glucose) was used to excise the amdSYM cassette from the genome of L69 generated mutants. For solid media, agar was added at $2 \%(\mathrm{w} / \mathrm{v})$ before sterilization at $120^{\circ} \mathrm{C}$ for $20 \mathrm{~min}$.

\section{Plasmids and strains construction}

To overexpress FLO11 gene or any of its alleles in yeast strain, expression vector pYES2.1 TOPO TA (Table S5) was used which carries GAL1 as promoter and CYC1 terminator. The FLO11 ORF from BY4741 (FLO11 $\left.{ }^{\mathrm{BY}}\right)$ and L69 $\left(\right.$ FLO11 $\left.{ }^{\text {L69 }}\right)$ were amplified by PCR using the primers FLO11_TOPO_f and FLO11_TOPO_r (Table S6) and ligated into pYES2.1 TOPO TA vector (ThermoFisher Scientific) between GAL1 promoter and CYC1 terminator according to the manufacture's protocol. Correct integration was confirmed by restriction digestion and plasmid sequencing.

Deletion of FLO11 were constructed in L69 strain using CRISPR-Cas9 strategy (Ryan et al., 2016). The high copy pCas9-amdSYM plasmid (derived from pML107, Addgene) that constitutively expresses the gene encoding Cas9 endonuclease protein and carrying a gRNA expression cassette (Sap1 cloning sites) was used. It confers the ability to S. cerevisiae to use acetamide as the sole nitrogen source (Solis-Escalante et al., 2013) enabling selection of prototroph strains. As the CRISPR/Cas9 technique requires the identification of a unique 20N (NGG) sequence, the candidate target sequence was identified using the online software CRISPR-direct (https://crispr.dbcls.jp) ), and determined as to be located in the middle of each region of FLO11 to be deleted. Linear healing fragments of $120 \mathrm{bp}$ were designed with $60 \mathrm{bp}$ overlapping the upstream and downstream sequences of the region to be deleted, namely the N-terminal (from 4 bp to $675 \mathrm{bp}$ ), the C-terminal (from 

new gRNA into pCas9-amdSYM was confirmed by Sanger sequencing using the M13 forward primer. The generated plasmids were used to transform L69 strain and healing fragments were added to repair the double PCR amplification and Sanger sequencing (Supplementary data, Table S6). and FLO11_BY_2964_r (see Table S3), whereas a 1794 bp fragment was PCR amplified from $F L O 11^{169}$ using the PCR amplified using FLO11_TOPO_f and FLO11_TOPO_r oligonucleotides (Supplementary data, Table S6). The resulting chimeric gene $F L O 11^{B Y}$ - $[R R 2]^{L 69}$ was ligated into PYES2.1 TOPO TA vector as described above.

\section{Bioinformatics}

The gene FLO11 was retrieved from the L69 strain genome which has been fully sequenced internally

578 (Lallemand, Inc, unpublished data) using the Pacific Biosciences method, whereas the sequences of FLO11 in

579 the laboratory strain BY4741 and $\Sigma 1278 \mathrm{~b}$ were uploaded from the SGD database

580 (https://www.yeastgenome.org). Clustal Omega was used to perform amino acid sequence alignments (Sievers

581 \& Higgins, 2014). Structural and functional comparison between the sequences of Flo11p ${ }^{\mathrm{L69}}$, Flo11p ${ }^{\mathrm{BY} 4741}$ and

582 Flo11 ${ }^{\Sigma 1278 b}$ were carried out using Hydrophobic Cluster Analysis (HCA) (Lemesle-Varloot et al., 1990) giving a

583 plot of each open reading frame that draws as a helical projection, vertically repeated. Secretion signal and the

584 GPI signal anchorage to cell wall $\beta$-glucan were searched using SignalP-4.1 server

585 (http://www.cbs.dtu.dk/services/SignalP-4.1/) (Nielsen, 2017), PredGPI tool

586 (http://gpcr.biocomp.unibo.it/predgpi/) (Pierleoni et al., 2008). TANGO software (http://tango.crg.es/) 
587 (Fernandez-Escamilla et al., 2004) with default settings for $\mathrm{pH}$, ionic strength, and temperature was used to

588 determine Flo11p regions with $\beta$-aggregation potential superior to $30 \%$. Intragenic repeats in the FLO11 ORF

were screened using the EMBOSS ETANDEM software (http://emboss.bioinformatics.nl/cgi-

bin/emboss/etandem) (Rice et al., 2000). Criteria were selected those with a repetition length $>10$, a score $>20$,

591 and a repetition conservation $>60 \%$.

592

593

594

595

\section{Atomic Force Microscopy}

Cells were collected from exponential growth, washed twice in acetate buffer (18 mM CH${ }_{3} \mathrm{COONa}, 1 \mathrm{mM}$ $\mathrm{CaCl}_{2}$ and, $1 \mathrm{mM} \mathrm{MnCl}, \mathrm{pH} 5.2$ ), and immobilized on polydimethylsiloxane (PDMS) stamps as described before in (Formosa et al., 2015). AFM experiments were recorded at room temperature using a Nanowizard III system (JPK-Bruker) and MLCT cantilevers (Bruker). The spring constants of each probe were systematically measured by the thermal noise method according to (Hutter \& Bechhoefer, 1993) and were found to be in the range of 0.01- $0.02 \mathrm{~N} \cdot \mathrm{m}^{-1}$. AFM height and adhesion images were recorded in Quantitative Imaging ${ }^{\mathrm{TM}}$ mode (JPK-Bruker) (Chopinet et al., 2013), and the maximal force applied to the cell was limited to $1 \mathrm{nN}$. For each condition, 3 independent experiments were performed and at least 12 cells were imaged. All results were analysed with the JPK Data Processing software. The adhesion values measured on cells were determined from the retract forcedistance curves. The stiffness values (kcell) were determined as the slope of the linear portion of the force versus indentation curves according to:

$$
k_{\text {cell }}=k\left(\frac{s}{1-s}\right)
$$

The effective spring constant of the cell $k_{\text {cell }}$ is calculated from the experimental slope $s$ of the force curve and the spring constant $k$ of the cantilever measured by the thermal noise method. All stiffness and adhesion values were considered for the histograms, which were generated using OriginPro version 2020 (OriginLab, Northampton, USA). A synthetic peptide based on the amyloid sequence VVSTTV bearing a replacement of $V$ by A (VASTTV) was purchased from Gencust and used as anti-amyloid peptide against Flo11p amyloid sequence. Cells were incubated for 90 minutes with $2 \mathrm{mg} / \mathrm{ml}$ of this peptide prior to AFM analysis.

\section{Quantitative Reverse Transcription PCR (RT-QPCR)}

Unless otherwise stated, yeast strains were cultured in YPD medium and 5 DO of exponentially growing cells ((O.D.600nm $\sim 1.5)$ were harvested by centrifugation, nitrogen frozen and stored at $-80^{\circ} \mathrm{C}$. RNA extraction was carried out using RNEasy Plus Mini kit (Qiagen) according to manufacturer's protocol. Quality 
and quantification of RNAs were determined using a Nanodrop 2000 (Thermo) and Bioanalyzer 2100 (Agilent).

$61710 \mu \mathrm{l}$ of iQ SYBR Green Supermix buffer (Bio-Rad), $5 \mu \mathrm{l}$ of Nuclease free water and $4 \mu \mathrm{l}$ of the appropriate

For determination of the adherence to polystyrene surfaces, the cells were grown overnight at $30^{\circ} \mathrm{C}$ in $\mathrm{YN}$ incubated at $30^{\circ} \mathrm{C}$ for $1 \mathrm{~h}$ at $200 \mathrm{rpm}$. An equal volume of $0.1 \%(\mathrm{w} / \mathrm{v})$ crystal violet was then added to each well. by solubilizing the retained crystal violet in $100 \mu \mathrm{L} 95 \%$ ethanol. After 10 min the absorbance of the samples against the blank was measured at $595 \mathrm{~nm}$ in microplate reader (Biotek).

Hydrophobic features of yeast surface were determined by measuring their affinity for a nonpolar solvent as described in (Purevdorj-Gage et al., 2007). Overnight cultures were centrifuged at $2.000 \mathrm{~g}$ for $5 \mathrm{~min}$ and resuspended in fresh $\mathrm{YN}$ Galactose medium at $\mathrm{OD}_{600}$ of 1 . After $3 \mathrm{~h}$ of static incubation at room temperature, O.D. ${ }_{600 \mathrm{~nm}}$ was measured $(A O)$ and $1.2 \mathrm{ml}$ of each culture was overlaid with $0.6 \mathrm{ml}$ of octane (Sigma-Aldrich) in 15 $\times 100 \mathrm{~mm}$ borosilicate glass tubes. The tubes were vigorously vortexed for $2 \mathrm{~min}$. and left on the bench for at least 15 min until complete separation of the two phases. A sample of the aqueous phase was taken with a

639 Pasteur pipette and the O.D. $600 \mathrm{~nm}$ was measured $(A)$. The results were expressed as the Octane adhesion index 640 (\% hydrophobicity), which represents the percentage of cells retained by the organic fraction, according to the equation: 
Invasive agar growth assay

Agar invasion assays were performed according to the method previously described (Roberts \& Fink, 1994). Strains were patched on glucose or galactose rich or synthetic medium plates supplemented with auxotrophic compounds when needed and grown at $30^{\circ} \mathrm{C}$ for 5 to 8 days. They were photographed before and after washing with distilled water.

\section{Cell-cell aggregation assay}

Cells were grown in YNGal medium and harvested during entry in stationary phase. Cell concentration of each sample was adjusted to $8.10^{7}$ cells. $\mathrm{mL}^{-1}$ and $4 \mu \mathrm{l}$ were dropped on a microscope slide and observed with a microscope Eclipse 400 (Nikon) after being briefly vortexed at low agitation. Aggregates with at least 5 cells were counted and reported as a percentage as previously described in (Purevdorj-Gage et al., 2007).

\section{Other phenotypic assays}

Sensitivity of yeast strains to caffeine (Sigma-Aldrich), calcofluor white (CFW; US Bio) and Congo Red (CR) was performed on YPD agar plates. Briefly, exponentially growing cells on YPD $\left(O . D_{600 \mathrm{~nm}}\right.$ around 1$)$ were collected by centrifugation and resuspended in sterilize water at $10 \mathrm{OD}$ units. Series of 10 -fold dilutions were spotted on YPD agar plates in the absence or presence of various concentrations of CFW or CR. Pictures were taken after 2 days of growth at $30^{\circ} \mathrm{C}$. Flocculation tests were carried out according to (Lo \& Dranginis, 1996), starting with overnight yeast cultures in YPD, washed once with deflocculating medium (20 mM citrate pH 3.0 containing $5 \mathrm{mM}$ EDTA), and resuspended in $1 \mathrm{ml}$ of the same solution at 1.0 unit $\mathrm{OD}_{600}$. Then, $\mathrm{CaCl} 2(1 \mathrm{M}$ solution) was added at a final concentration of $20 \mathrm{mM}$ and decrease of absorbance was monitored at $600 \mathrm{~nm}$. Velum formation was carried out exactly as described in Zara et al. (Zara et al., 2005). The flor yeast strain A9 (kind gift from M. Budroni, Univ Sassari, Italy) was used as a control.

\section{Statistical analysis}

All phenotypic assays were carried out at least 3 times with independent biological samples. Statistical analysis were made by one-way analysis of variance followed by Tukey's test on Microsoft Excel software. Statistical significant values were denoted by asterisks on the figures as $*=p$-value $<0.05, * *=p$-value $<0.01$ and $* * *=p$-value $<0.001$. 
669 The authors declare no commercial or financial conflict of interest.

670

671

672

673

674

675

676

677

678

679

680

681

682

683

684

685

686

687

688

689

690

691

692

693

694

695

696

697

698

699

700

701

702

703

704

705

706

\section{FUNDING}

This work was supported in part by a grant from Lallemand Inc. (project Lallwall, nºAIC2016/048 \& SAIC/2018/010) and by part by Region Midi Pyrénées, grant Nº9003813 to JMF

\section{ACKNOWLEDGEMENTS}

We are grateful Dr Jean Luc Parrou for advice on performing RT-qPCR experiments, to Dr Charlie Boone of University Toronto Canada, and Dr Marilena Budroni from University of Sassari, Italy, for the kind gift of yeast strains, and to Dr Mathieu Castex from Lallemand Inc. for continuous support on this work. CB is financed by ANRT (Agence Nationale de la Recherche et des Technologies) grant to carry out her PhD thesis.

\section{REFERENCES}

Alexandre $\mathrm{H}$ (2013) Flor yeasts of Saccharomyces cerevisiae--their ecology, genetics and metabolism. Int J Food Microbiol 167: 269-275. S0168-1605(13)00407-8 [pii];10.1016/j.ijfoodmicro.2013.08.021 [doi].

Alsteens D, Garcia MC, Lipke PN \& Dufrene YF (2010) Force-induced formation and propagation of adhesion nanodomains in living fungal cells. Proc Natl Acad Sci U S A 107: 20744-20749. 1013893107 [pii];10.1073/pnas.1013893107 [doi].

Barua S, Li L, Lipke PN \& Dranginis AM (2016) Molecular Basis for Strain Variation in the Saccharomyces cerevisiae Adhesin Flo11p. mSphere 1. 10.1128/mSphere.00129-16 [doi];mSphere00129-16 [pii].

Bruckner S, Schubert R, Kraushaar T, Hartmann R, Hoffmann D, Jelli E, Drescher K, Muller DJ, Oliver Essen L \& Mosch HU (2020) Kin discrimination in social yeast is mediated by cell surface receptors of the Flo11 adhesin family. Elife 9. 10.7554/eLife.55587.

Chan CX \& Lipke PN (2014) Role of force-sensitive amyloid-like interactions in fungal catch bonding and biofilms. Eukaryot Cell 13: 1136-1142. 10.1128/EC.00068-14.

Chan CX, El-Kirat-Chatel S, Joseph IG, Jackson DN, Ramsook CB, Dufrene YF \& Lipke PN (2016) Force Sensitivity in Saccharomyces cerevisiae Flocculins. mSphere 1. 10.1128/mSphere.00128-16.

Chopinet L, Formosa C, Rols MP, Duval RE \& Dague E (2013) Imaging living cells surface and quantifying its properties at high resolution using AFM in QI mode. Micron 48: 26-33.

Dague E, Alsteens D, Latge JP, Verbelen C, Raze D, Baulard AR \& Dufrene YF (2007) Chemical force microscopy of single live cells. Nano Lett 7: 3026-3030.

Dowell RD, Ryan O, Jansen A, et al. (2010) Genotype to phenotype: a complex problem. Science 328: 469. 10.1126/science.1189015.

Dranginis AM, Rauceo JM, Coronado JE \& Lipke PN (2007) A biochemical guide to yeast adhesins: glycoproteins for social and antisocial occasions. Microbiol Mol Biol Rev 71: 282-294.

Fernandez-Escamilla AM, Rousseau F, Schymkowitz J \& Serrano L (2004) Prediction of sequence-dependent and mutational effects on the aggregation of peptides and proteins. Nat Biotechnol 22: 1302-1306. $10.1038 / \mathrm{nbt} 1012$.

Fidalgo M, Barrales RR, Ibeas JI \& Jimenez J (2006) Adaptive evolution by mutations in the FLO11 gene. Proc Natl Acad Sci U S A 103: 11228-11233. 0601713103 [pii];10.1073/pnas.0601713103 [doi]. 
Formosa C, Schiavone M, Boisrame A, Richard ML, Duval RE \& Dague E (2015) Multiparametric imaging of adhesive nanodomains at the surface of Candida albicans by atomic force microscopy. Nanomedicine 11: 57-65. S1549-9634(14)00418-3 [pii];10.1016/j.nano.2014.07.008 [doi].

Formosa C, Pillet F, Schiavone M, Duval RE, Ressier L \& Dague E (2015) Generation of living cell arrays for atomic force microscopy studies. Nat Protoc 10: 199-204. nprot.2015.004 [pii];10.1038/nprot.2015.004 [doi].

Free SJ (2013) Fungal cell wall organization and biosynthesis. Adv Genet 81: 33-82.

Gancedo JM (2001) Control of pseudohyphae formation in Saccharomyces cerevisiae. FEMS Microbiol Rev 25: 107-123.

Garcia MC, Lee JT, Ramsook CB, Alsteens D, Dufrene YF \& Lipke PN (2011) A role for amyloid in cell aggregation and biofilm formation. PLoS One 6: e17632. 10.1371/journal.pone.0017632.

Gietz RD \& Schiestl RH (2007) High-efficiency yeast transformation using the LiAc/SS carrier DNA/PEG method. Nat Protoc 2: 31-34. 10.1038/nprot.2007.13.

Goffeau A (1998) The yeast genome. Pathol Biol (Paris) 46: 96-97.

Guo B, Styles CA, Feng Q \& Fink GR (2000) A Saccharomyces gene family involved in invasive growth, cell-cell adhesion, and mating. Proc Natl Acad Sci U S A 97: 12158-12163.

Hutter JI \& Bechhoefer J (1993) Calibration of atomic force microscope tips. Rev Scientific Instruments 64: 18681873.

Klis FM, Boorsma A \& de Groot PW (2006) Cell wall construction in Saccharomyces cerevisiae. Yeast 23: 185202.

Kobayashi O, Yoshimoto H \& Sone H (1999) Analysis of the genes activated by the FLO8 gene in Saccharomyces cerevisiae. Curr Genet 36: 256-261. 10.1007/s002940050498.

Kraushaar T, Bruckner S, Veelders M, Rhinow D, Schreiner F, Birke R, Pagenstecher A, Mosch HU \& Essen LO (2015) Interactions by the Fungal Flo11 Adhesin Depend on a Fibronectin Type III-like Adhesin Domain Girdled by Aromatic Bands. Structure 23: 1005-1017. 10.1016/j.str.2015.03.021.

Lemesle-Varloot L, Henrissat B, Gaboriaud C, Bissery V, Morgat A \& Mornon JP (1990) Hydrophobic cluster analysis: procedures to derive structural and functional information from 2-D-representation of protein sequences. Biochimie 72: 555-574. 10.1016/0300-9084(90)90120-6.

Levin DE (2005) Cell Wall Integrity Signaling in Saccharomyces cerevisiae. Microbiol Mol Biol Rev 69: 262-291.

Lipke PN, Klotz SA, Dufrene YF, Jackson DN \& Garcia-Sherman MC (2018) Amyloid-Like beta-Aggregates as Force-Sensitive Switches in Fungal Biofilms and Infections. Microbiol Mol Biol Rev 82. 10.1128/MMBR.00035-17.

Lipke PN, Garcia MC, Alsteens D, Ramsook CB, Klotz SA \& Dufrene YF (2012) Strengthening relationships: amyloids create adhesion nanodomains in yeasts. Trends Microbiol 20: 59-65.

Liu H, Styles CA \& Fink GR (1996) Saccharomyces cerevisiae S288C has a mutation in FLO8, a gene required for filamentous growth. Genetics 144: 967-978.

Livak KJ \& Schmittgen TD (2001) Analysis of relative gene expression data using real-time quantitative PCR and the 2(-Delta Delta C(T)) Method. Methods 25: 402-408. 10.1006/meth.2001.1262.

Lo WS \& Dranginis AM (1996) FLO11, a yeast gene related to the STA genes, encodes a novel cell surface flocculin. J Bacteriol 178: 7144-7151.

Lo WS \& Dranginis AM (1998) The cell surface flocculin Flo11 is required for pseudohyphae formation and invasion by Saccharomyces cerevisiae. Mol Biol Cell 9: 161-171.

Mans R, van Rossum HM, Wijsman M, Backx A, Kuijpers NG, van den Broek M, Daran-Lapujade P, Pronk JT, van Maris AJ \& Daran JM (2015) CRISPR/Cas9: a molecular Swiss army knife for simultaneous introduction of multiple genetic modifications in Saccharomyces cerevisiae. FEMS Yeast Res 15 . fov004 [pii];10.1093/femsyr/fov004 [doi].

Meem MH \& Cullen PJ (2012) The impact of protein glycosylation on Flo11-dependent adherence in Saccharomyces cerevisiae. FEMS Yeast Res 12: 809-818. 10.1111/j.1567-1364.2012.00832.x [doi]. 
Mortensen HD, Dupont K, Jespersen L, Willats WG \& Arneborg N (2007) Identification of amino acids involved in the Flo11p-mediated adhesion of Saccharomyces cerevisiae to a polystyrene surface using phage display with competitive elution. J App/ Microbiol 103: 1041-1047. 10.1111/j.1365-2672.2007.03325.x.

Nielsen H (2017) Predicting Secretory Proteins with SignalP. Methods Mol Biol 1611: 59-73. 10.1007/978-14939-7015-5_6.

Orlean P (2012) Architecture and biosynthesis of the Saccharomyces cerevisiae cell wall. Genetics 192: 775-818.

Otoo HN, Lee KG, Qiu W \& Lipke PN (2008) Candida albicans Als adhesins have conserved amyloid-forming sequences. Eukaryot Cell 7: 776-782. 10.1128/EC.00309-07.

Pierleoni A, Martelli PL \& Casadio R (2008) PredGPI: a GPI-anchor predictor. BMC Bioinformatics 9: 392. 10.1186/1471-2105-9-392.

Purevdorj-Gage B, Orr ME, Stoodley P, Sheehan KB \& Hyman LE (2007) The role of FLO11 in Saccharomyces cerevisiae biofilm development in a laboratory based flow-cell system. FEMS Yeast Res 7: 372-379. FYR189 [pii];10.1111/j.1567-1364.2006.00189.x [doi].

Ramsook CB, Tan C, Garcia MC, et al. (2010) Yeast cell adhesion molecules have functional amyloid-forming sequences. Eukaryot Cell 9: 393-404. EC.00068-09 [pii];10.1128/EC.00068-09 [doi].

Reynolds TB \& Fink GR (2001) Bakers' yeast, a model for fungal biofilm formation. Science 291: 878-881. $10.1126 /$ science.291.5505.878.

Rice P, Longden I \& Bleasby A (2000) EMBOSS: the European Molecular Biology Open Software Suite. Trends Genet 16: 276-277. 10.1016/s0168-9525(00)02024-2.

Roberts RL \& Fink GR (1994) Elements of a single MAP kinase cascade in Saccharomyces cerevisiae mediate two developmental programs in the same cell type: mating and invasive growth. Genes Dev 8: 2974-2985.

Ryan OW, Poddar S \& Cate JH (2016) CRISPR-Cas9 Genome Engineering in Saccharomyces cerevisiae Cells. Cold Spring Harb Protoc 2016. 10.1101/pdb.prot086827.

Schiavone M, Sieczkowski N, Castex M, Dague E \& Marie Francois J (2015) Effects of the strain background and autolysis process on the composition and biophysical properties of the cell wall from two different industrial yeasts. FEMS Yeast Res 15. 10.1093/femsyr/fou012.

Sievers F \& Higgins DG (2014) Clustal omega. Curr Protoc Bioinformatics 48: 3 13 $11-16$. 10.1002/0471250953.bi0313s48.

Solis-Escalante D, Kuijpers NG, Bongaerts N, Bolat I, Bosman L, Pronk JT, Daran JM \& Daran-Lapujade P (2013) amdSYM, a new dominant recyclable marker cassette for Saccharomyces cerevisiae. FEMS Yeast Res 13: 126-139. 10.1111/1567-1364.12024 [doi].

Strahl-Bolsinger S, Gentzsch M \& Tanner W (1999) Protein O-mannosylation. Biochim Biophys Acta 1426: $297-$ 307.

Teste MA, Duquenne M, Francois JM \& Parrou JL (2009) Validation of reference genes for quantitative expression analysis by real-time RT-PCR in Saccharomyces cerevisiae. BMC Mol Biol 10: 99. 10.1186/14712199-10-99.

Verstrepen KJ \& Klis FM (2006) Flocculation, adhesion and biofilm formation in yeasts. Mol Microbiol 60: 5-15.

Voordeckers K, Kominek J, Das A, et al. (2015) Adaptation to High Ethanol Reveals Complex Evolutionary Pathways. PLoS Genet 11: e1005635. 10.1371/journal.pgen.1005635 [doi];PGENETICS-D-15-01464 [pii].

Zara G, Zara S, Pinna C, Marceddu S \& Budroni M (2009) FLO11 gene length and transcriptional level affect biofilm-forming ability of wild flor strains of Saccharomyces cerevisiae. Microbiology 155: 3838-3846. mic.0.028738-0 [pii];10.1099/mic.0.028738-0 [doi].

Zara S, Bakalinsky AT, Zara G, Pirino G, Demontis MA \& Budroni M (2005) FLO11-Based Model for Air-Liquid Interfacial Biofilm Formation by Saccharomyces cerevisiae. Appl Environ Microbiol 71: 2934-2939. 
bioRxiv preprint doi: https://doi.org/10.1101/2021.04 01.438097: this version posted April 5, 2021. The copyright holder for this preprint (which was not certified by peer review) is the author/funder, who has granted bioRxiv a license to display the preprint in perpetuity. It is made available under aCC-BY 4.0 International license.

Table 1. $\beta$-aggregation prone sequences in Flo11 of various Saccharomyces cerevisiae strains*

\begin{tabular}{lccc} 
& Amyloid sequence & Amino-acid position & $\% \beta$ aggregation \\
\hline Flo11p $^{\text {L69 }}$ & VVSTTVV & $1178 ; 1285 ; 1392$ & 75.7 \\
& VTTAVT & 1494 & 59.4 \\
Flo11p $^{\text {BY }}$ & VVSTTVV & 1033 & 75.8 \\
& VTTAVT & 1494 & 84.8 \\
Flo11p $^{\Sigma}$ & VVSTTVV & & 75.6 \\
& VTTAVT & 881 & 59 \\
Flo11p $^{133 d}$ & VVSTTVV & 983 & 70.9 \\
& VTTAVT & 1301 & 59.1 \\
\hline
\end{tabular}


Figure 1: Hydrophobic-cluster analysis (HCA) of flocculins from the yeast S288C. The analysis highlights domain structure and patterns repeats. The primary structure of all Flo proteins encompasses 3 domains, namely $\mathrm{N}$-terminal A domain with a secretion signal boxed in blue, a central Ser-Thr rich B-domain that carries repeat sequences with a $\beta$-aggregation potential superior to $70 \%$ marked by blue triangle, and a C-terminal domain that carries a GPI anchoring site shown by a green box and containing a highly hydrophobic sequence indicated by a red triangle.

Figure 2: Cell surface analysis of the L69 strain using silicon-nitride (Si3N4) AFM cantilevers. AFM height image (A, B) and adhesion image (D) of a single yeast cell from L69 strain are shown. In (B) is reported an AFM contact image in which is pointed out a specific area with an arrow from which has been obtained the topography data reported in (C). In (E) are represented typical force-distance curve from high adhesive and low adhesion force and illustrated how stiffness was determined on the curves. In (F) is reported the adhesion force histogram obtained from 1024 force-distance curve recorded in QI ${ }^{\mathrm{TM}}$ mode. Cell stiffness is represented by AFM image (G) and its quantification (H) from 1024 force-distance curve recorded in Q ${ }^{\mathrm{TM}}$ mode.

Figure 3: The nanosized patches at the cell surface of L69 strain are FLO11-dependent adhesion nanodomains flo11 1 mutant from L69 strain and of the laboratory BY4741 strain deleted of its endogenous FLO11 gene

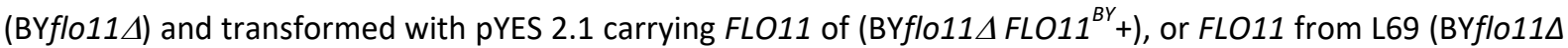
$F L O 11^{169}+$ ) are shown in panel A. In panel B is shown the AFM adhesion image of a cell from L69 strain before and $90 \mathrm{~min}$ after treatment with $5 \mu \mathrm{M}$ of the anti-amyloid peptide VASTTV. In panel $\mathrm{C}$ is shown the adhesion image of a cell from a culture of $L 69$ strain $\left(10^{7}\right.$ cells $\left./ \mathrm{ml}\right)$ before and after $30 \mathrm{~min}$ of incubation with $10 \mu \mathrm{M}$ of the anti-amyloid dye thioflavin S.

Figure 4: Hydrophobic-cluster analysis of the Flo11 protein from various Saccharomyces cerevisiae strain, and quantitative RT-PCR of wild type and mutated FLO11 genes. In (A), is reported the HCA plots of Flo11p from proteins as determined using various software as described in Material \& Methods are highlighted by A, B and 
amyloid-forming sequences are indicated in yellow for 'VVSTTV' and red for 'VTTAVT'. Pink and purple boxes three strains. The C-terminal GPI signal is boxed in green with a blue line indicating the omega-site position (GPI signal anchorage to cell wall $\beta$-glucan). Grey arrows delimit the $\mathrm{N}$-terminal, RR1, RR2 and C-terminal domains. In (B), is reported the quantitative expression levels of the different FLO11 alleles encoding the corresponding Flo11 protein variants relative to the expression level of wild type FLO11 in L69 strain. Samples for this qRT-PCR were taken in exponential growth phase on YN Galactose medium. Normalization of transcripts was done using TAF10 and UBC6 as internal reference as described in Material and Methods. defective of the N-terminus (flo11- $\Delta$ Nter), C-terminus (flo11- $\Delta$ Cter) or removed from the RR1 (flo11-ARR1) or RR2 (flo11- $\triangle R R 2$ ) domain as depicted in Fig 3A. In (B) is shown AFM height and adhesion images of a single cell from BYflo11 1 strain transformed with pYES2.1 carrying the chimeric $F L O 11^{B Y}[R R 2]^{L 69}$ gene that corresponded to wild type FLO11 of BY4741 in which RR2 sequence from FLO11 of strain L69 has been inserted.

Figure 6: The Flo11p cell-cell aggregation is potentiated by increase number of amyloid-forming sequence. microscope. In (A) are shown representative photographs of cell aggregates from the various strains studied. In (B) is represented for each strain the percentage of aggregates that are formed by at least 5 or more cells. For each strain, more than 100 cells or aggregates were counted under the microscope. Values shown are the mean of three biological replicates and vertical bars represent standard deviations. Significant differences are denoted with asterisks $\left(*=p\right.$-value $<0.05 ;{ }^{* *}=p$-value $\leq 0.01 ; * * *=p$-value $\left.\leq 0.005\right)$

Figure 7: Partial Flo11p and pH-dependencies of adherence to plastic. Adherence of yeast cells was carried out

856 a 96-well polystyrene plate as described in Methods. The data are the mean of three replicates measurements $857 \pm$ standard deviation. Significant differences are denoted with asterisks $\left(*=p\right.$-value $<0.05 ;{ }^{* *}=p$ value $\leq 0.01 ;^{* * *}=p$-value $\left.\leq 0.005\right)$

859 Figure 8: Surface hydrophobicity mainly depends on abundance of Flo11p. Surface hydrophobicity 

made available under aCC-BY 4.0 International license.

861 the mean of three biological replicates and vertical bars represent standard deviations. Significant differences

862 are denoted with asterisks $(*=p$-value $<0.05 ; * *=p$-value $\leq 0.01 ; * * *=p$-value $\leq 0.005)$.

863 Figure 9: the Invasive growth in agar is abolished by deletion of either $\mathbf{N}$ or C-terminal of the Flo11p. All

864 strains were pre-grown in YNGal that was supplemented with the auxotrophic requirements when needed (ie

865 uracil, leucine, histidine, methionine at 0.1\% for BY4741, BYflo11 and YSTW3 $\alpha$ but only leucine, histidine,

866 methionine for BYflo11 $\Delta$ and $\Sigma$ flo11 1 expressing $F L O 11^{B Y}, F L O 11^{L 69}$ or $F L O 11^{B Y}$ - $[R R 2]^{L 69}$ ) until stationary phase.

867 Then, $10 \mu \mathrm{L}$ of these cultures were spotted on $2 \%(\mathrm{w} / \mathrm{v})$ agar plate made with YPD (Panel A) or YNGal

868 complemented with auxotrophic requirements (panel B). Plates were incubated at $30^{\circ} \mathrm{C}$ for 8 days and washed

869 under a stream of water. They were photographed before and after washing.

870 


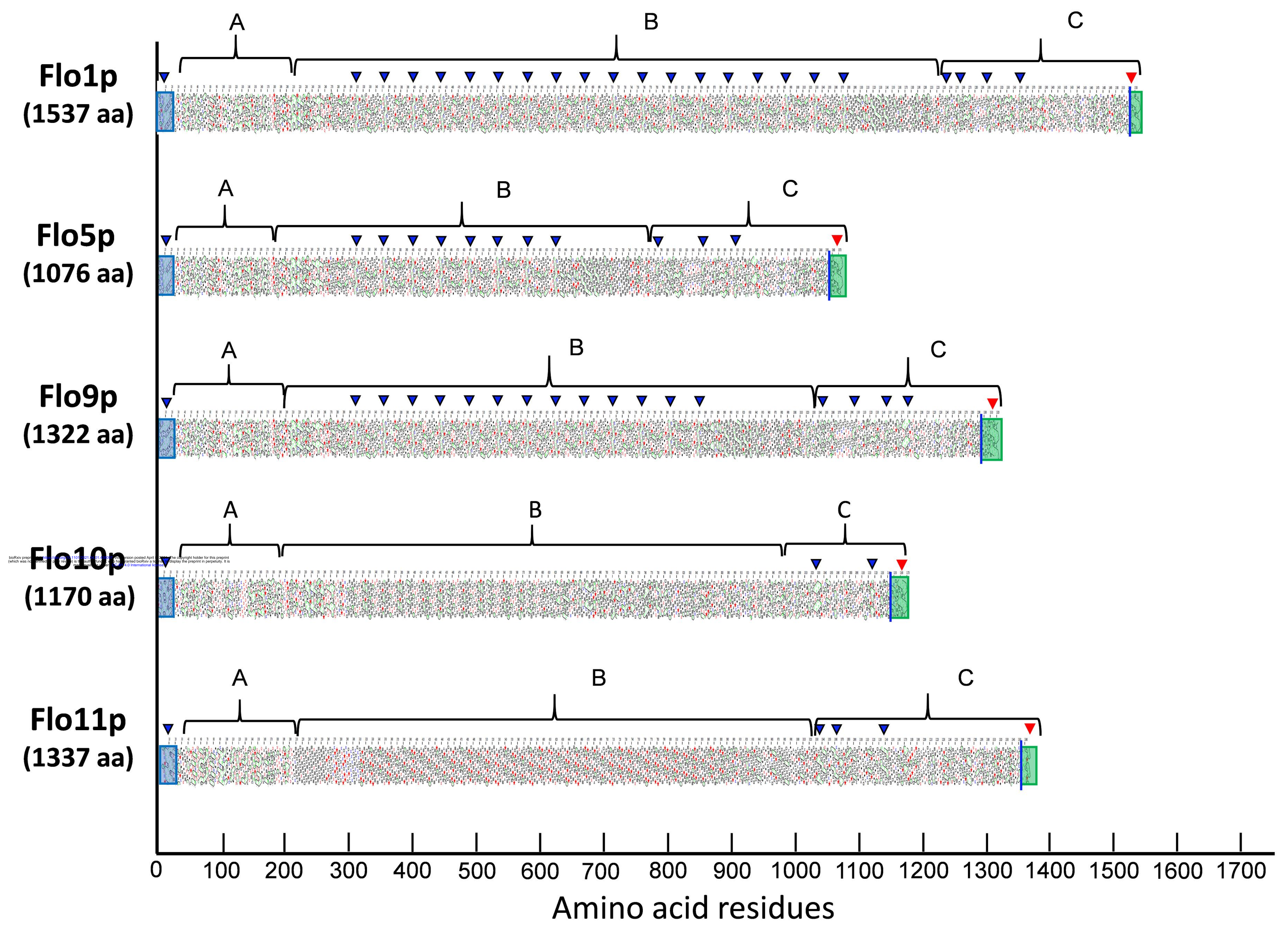


A
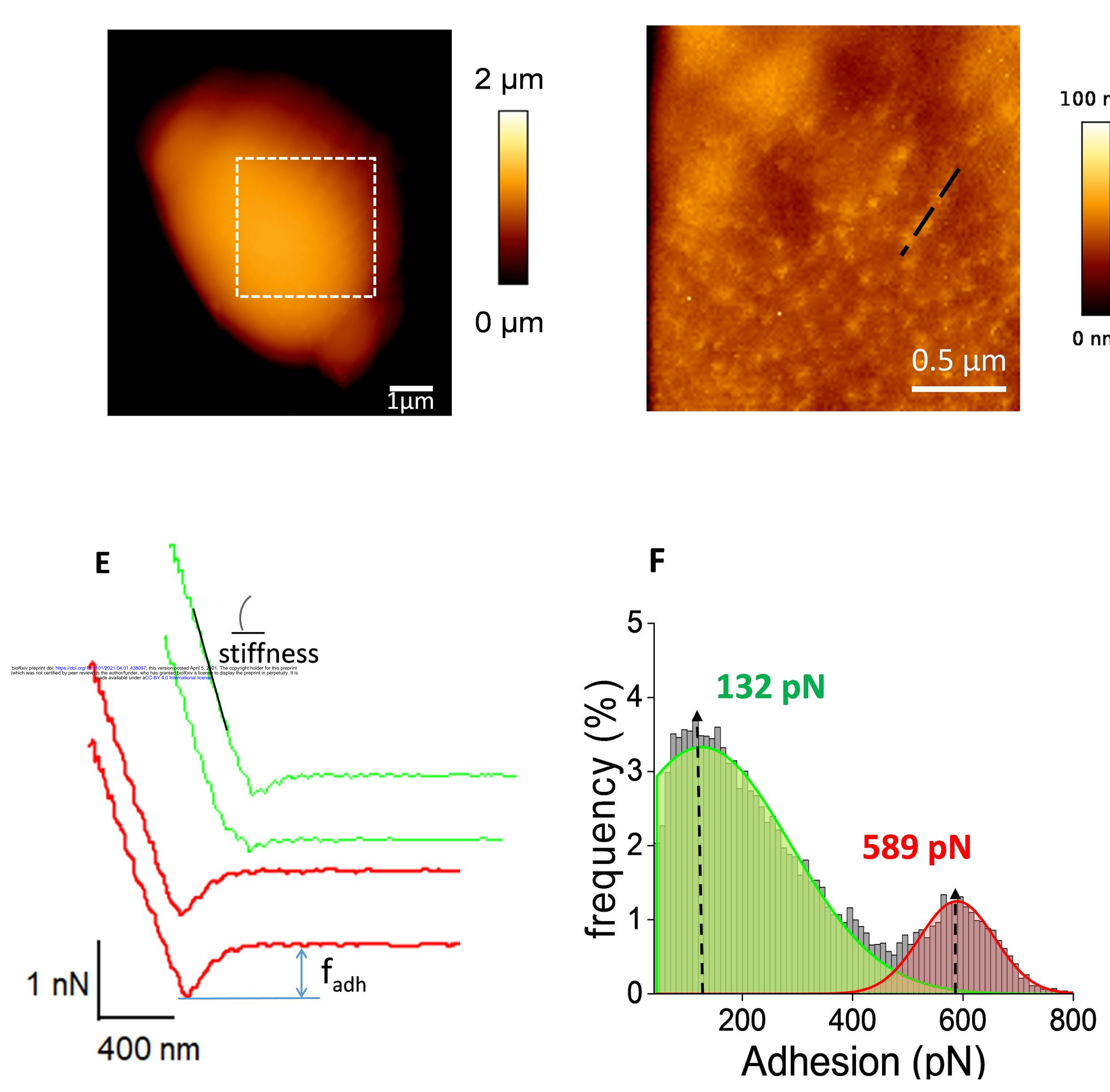

C

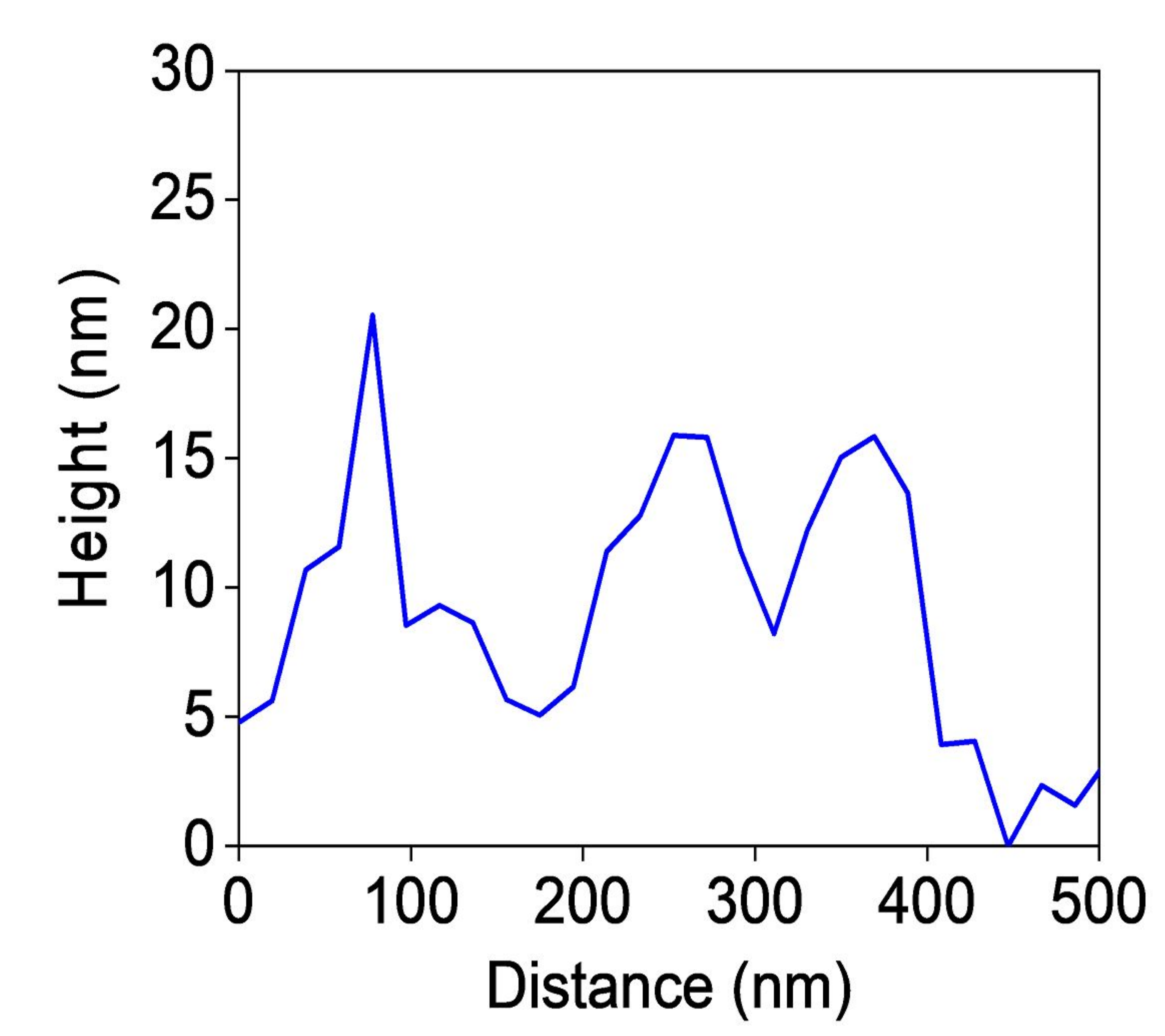

D

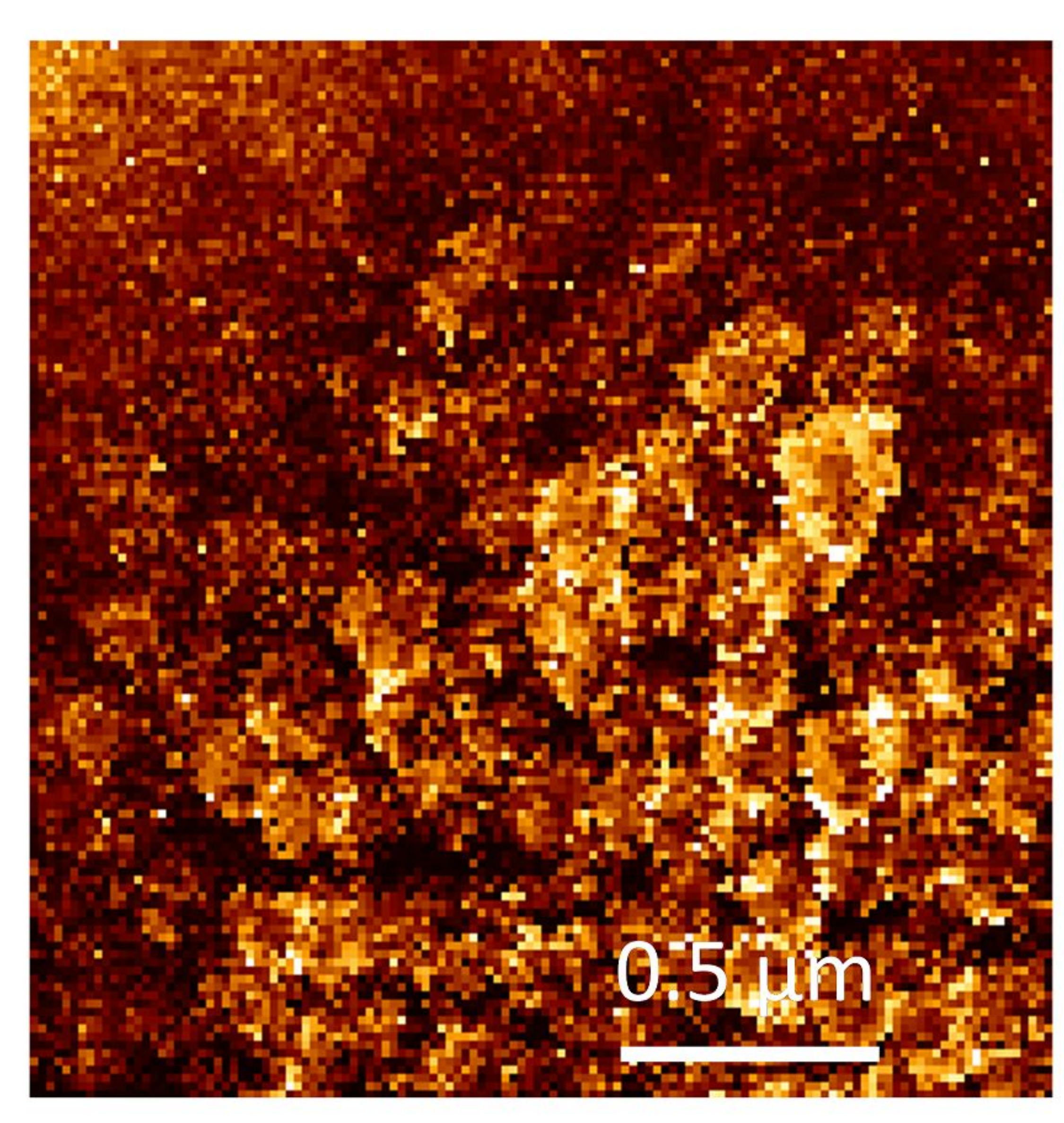

$800 \mathrm{pN}$

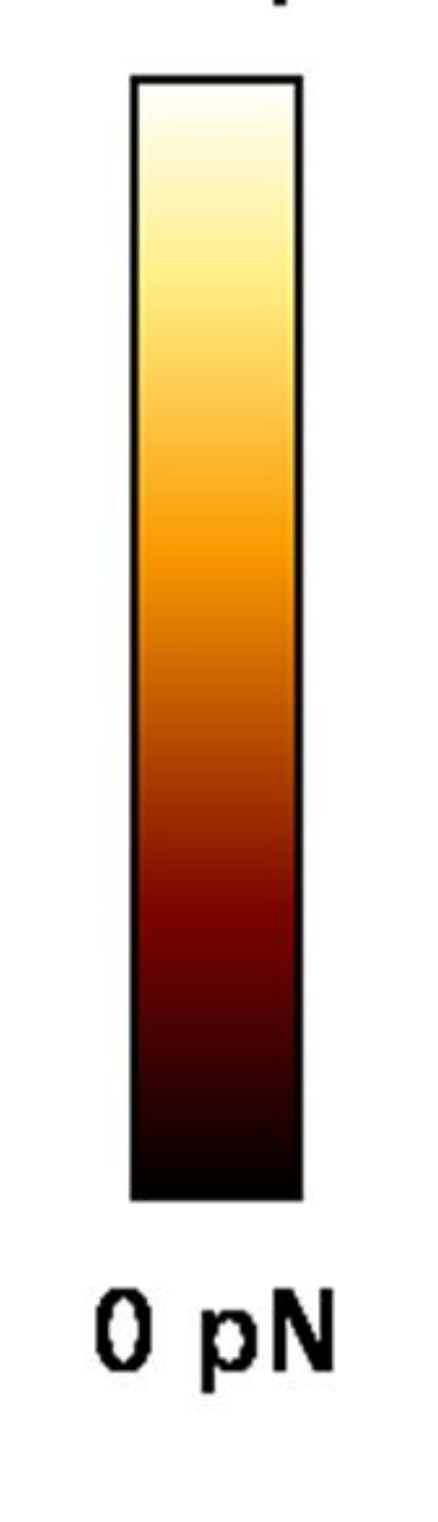

G
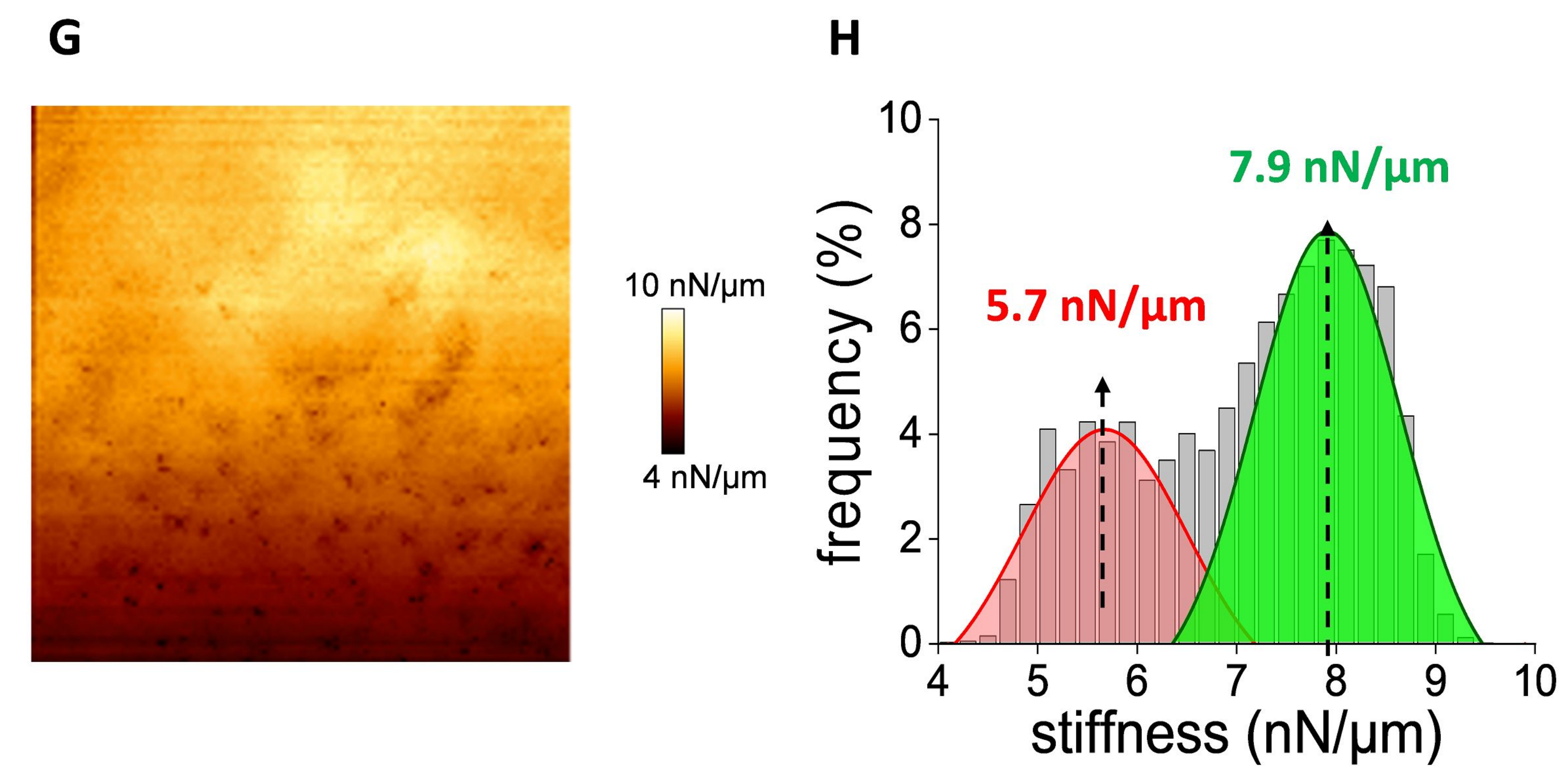

stiffness (nN/ $/ \mu \mathrm{m})$ 
A T $0 \mathrm{~min}$
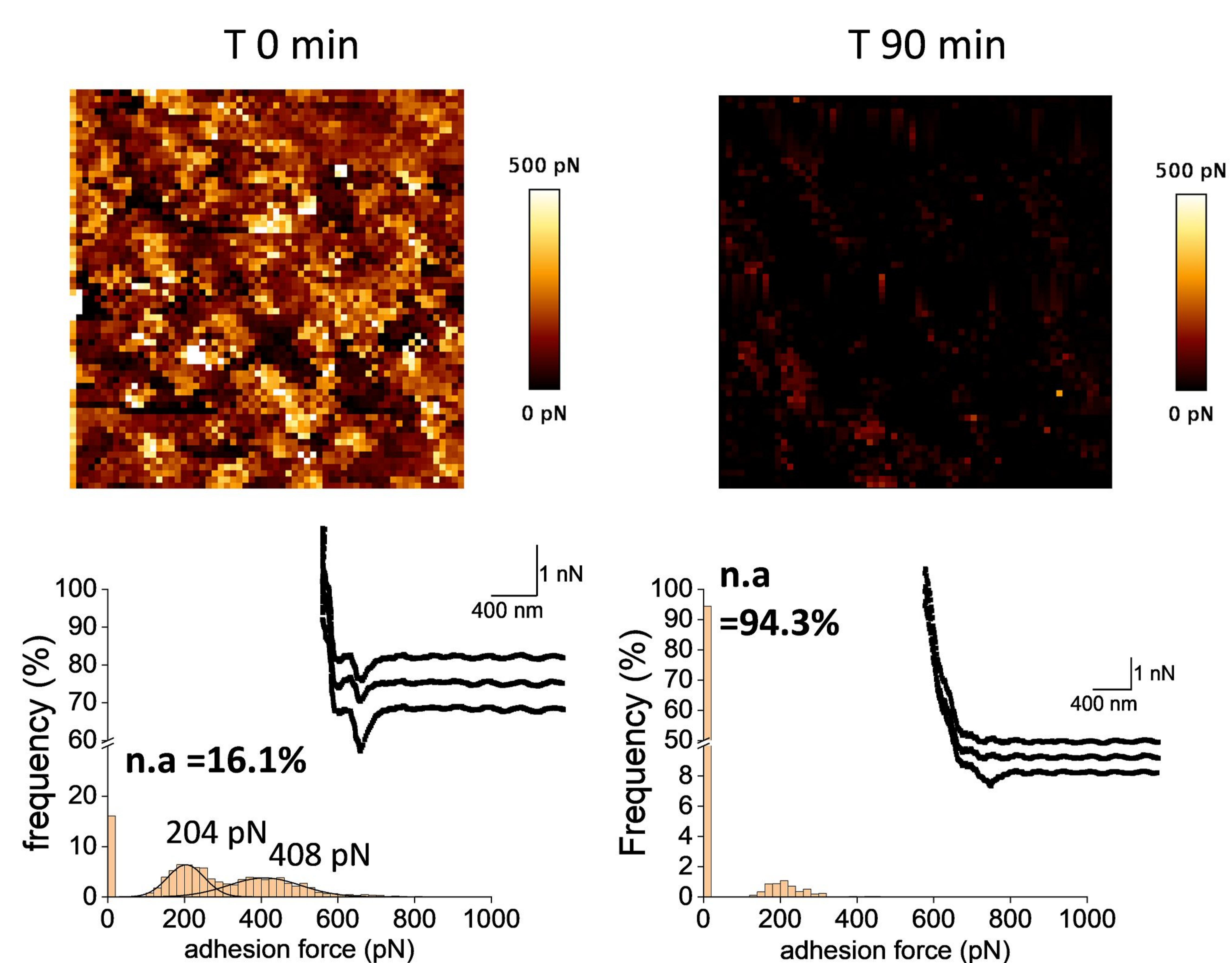

B
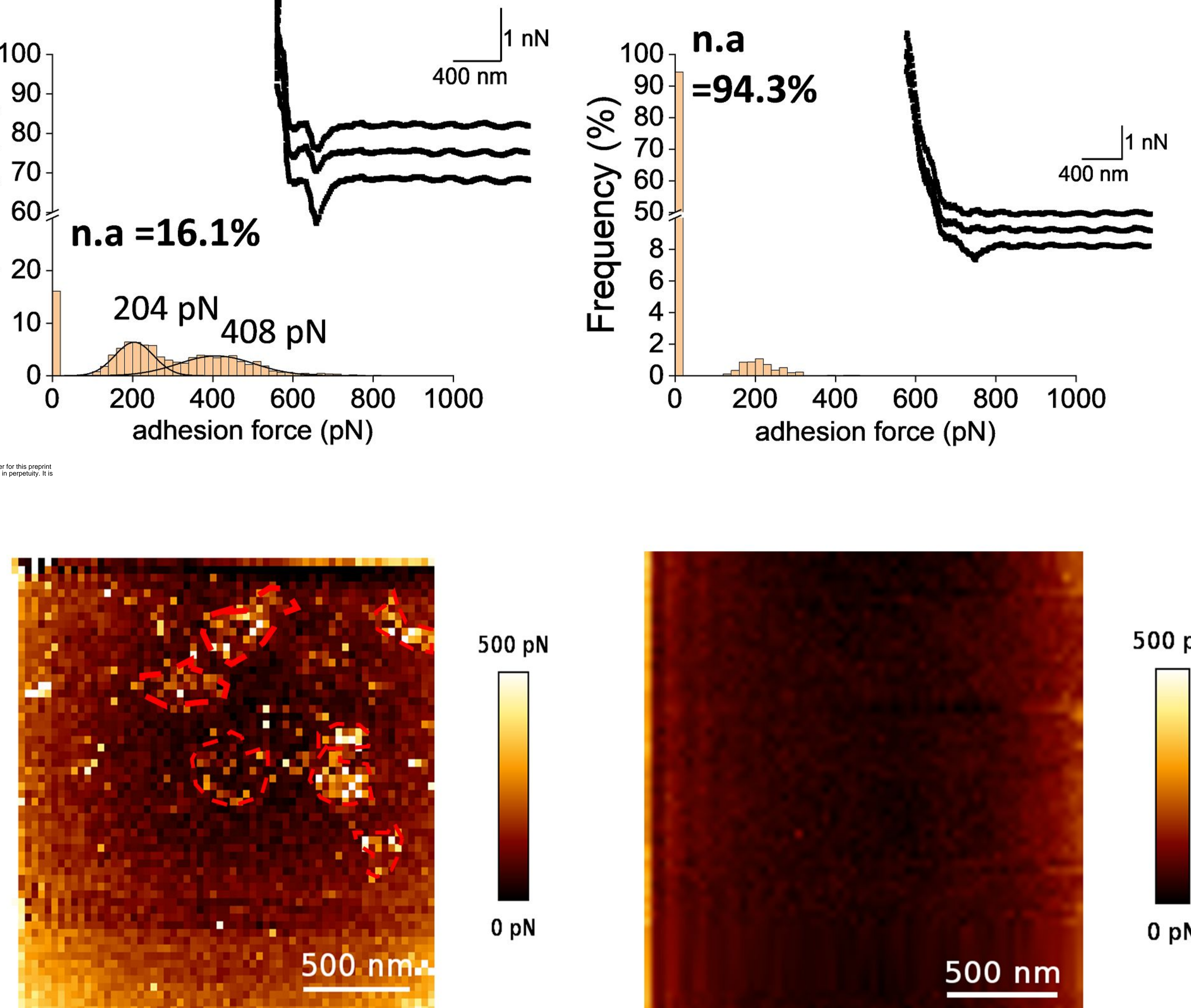

C

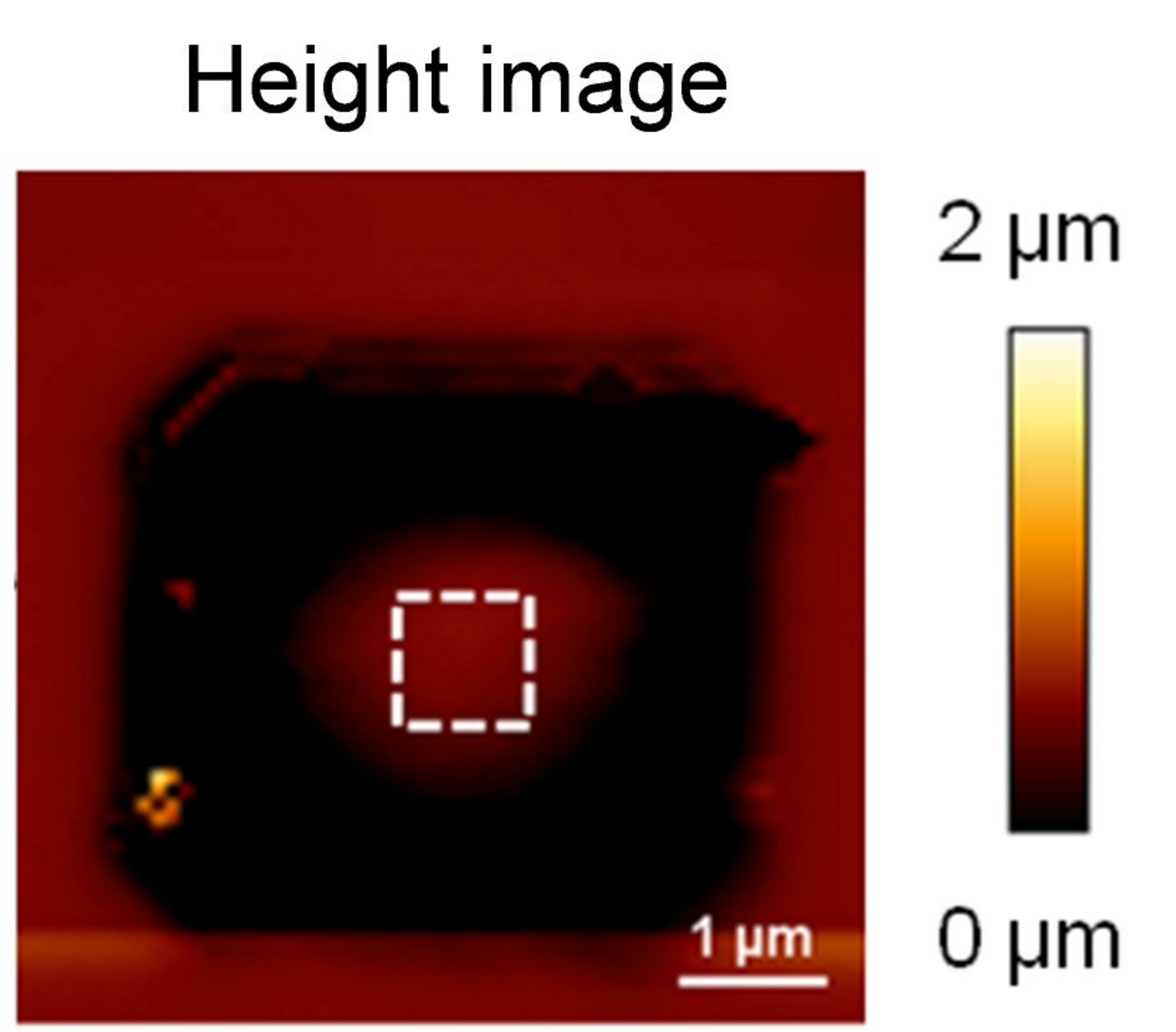

L69flo11 $\Delta$
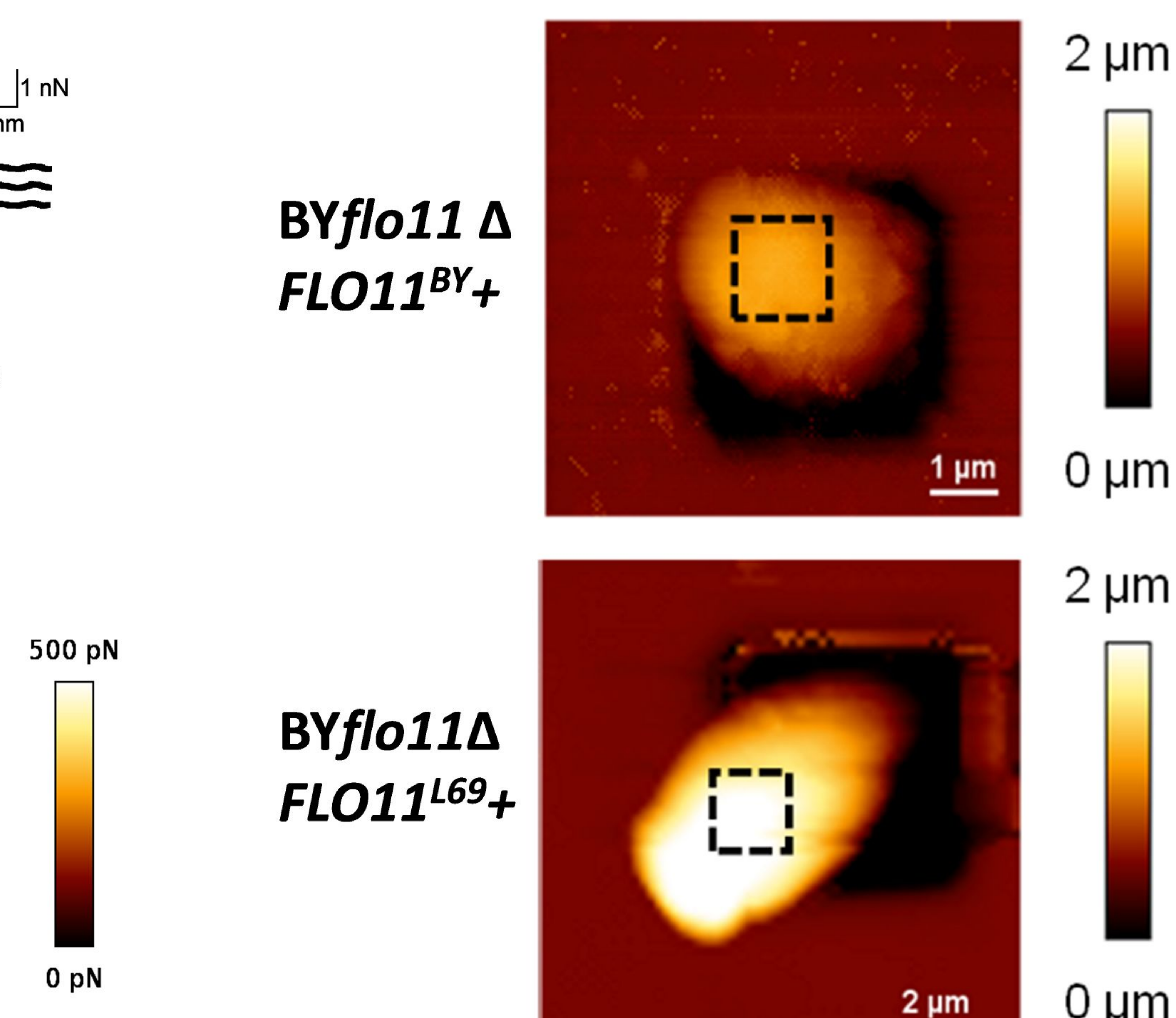

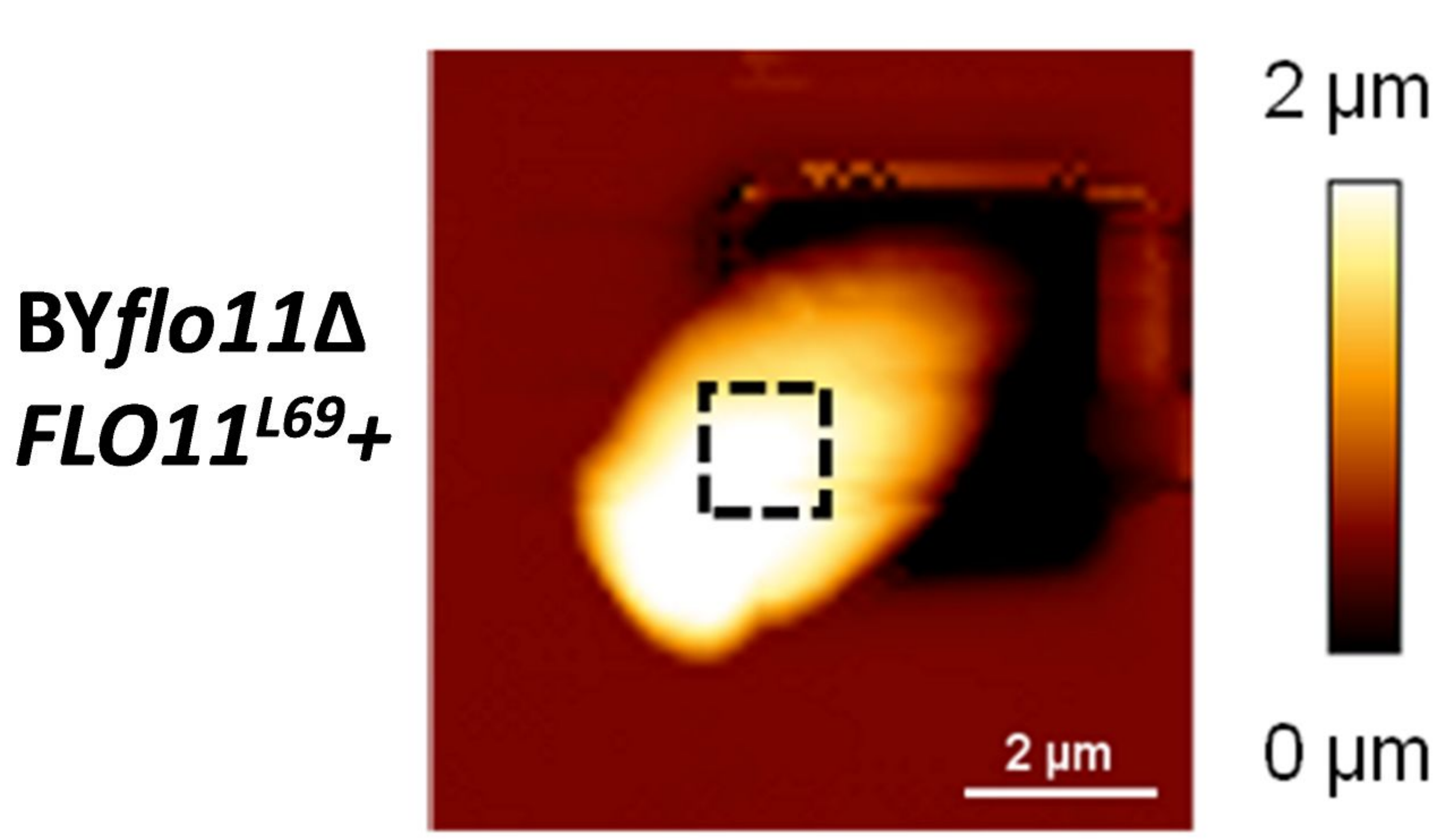

adhesion image
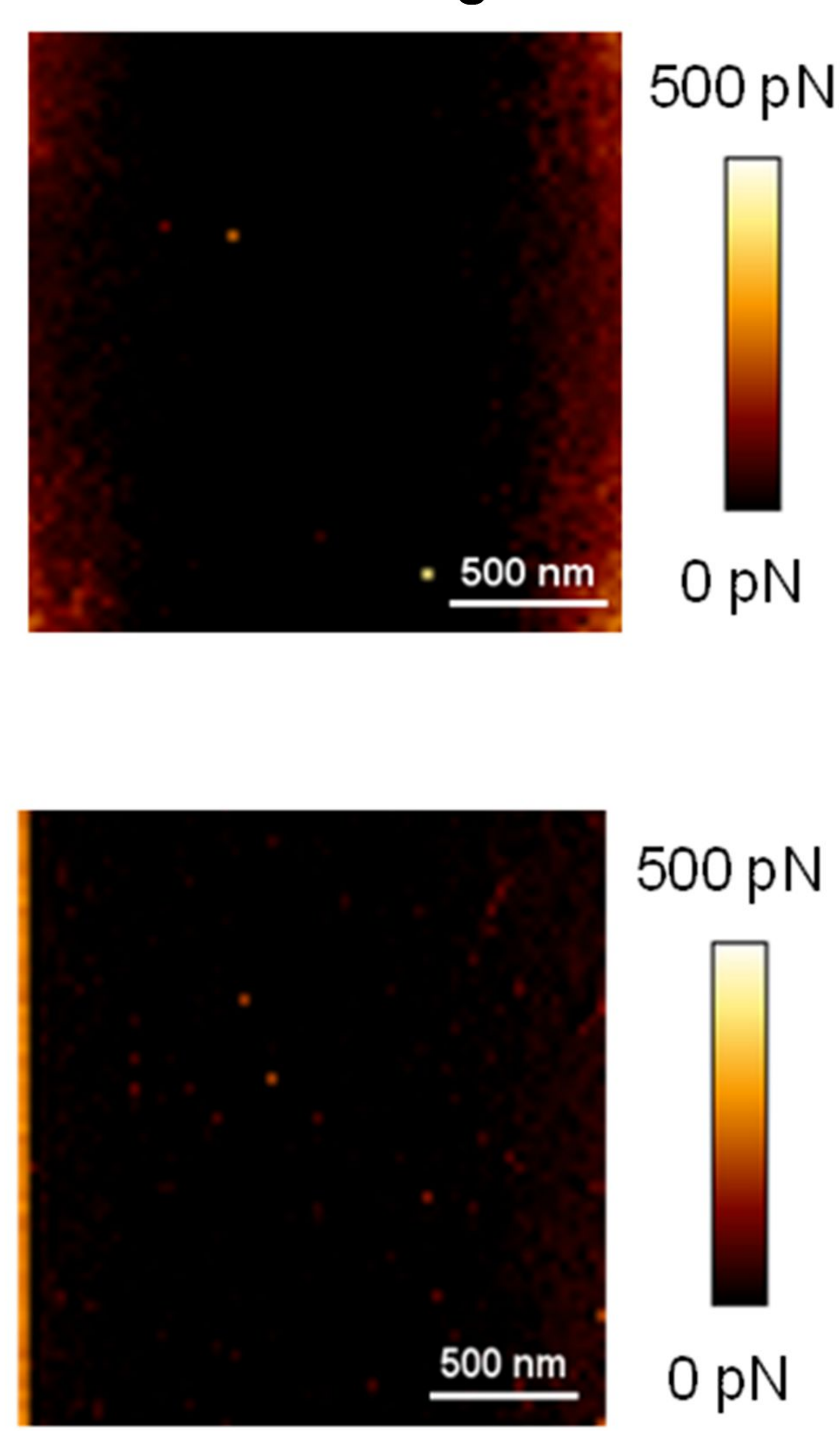

$500 \mathrm{pN}$
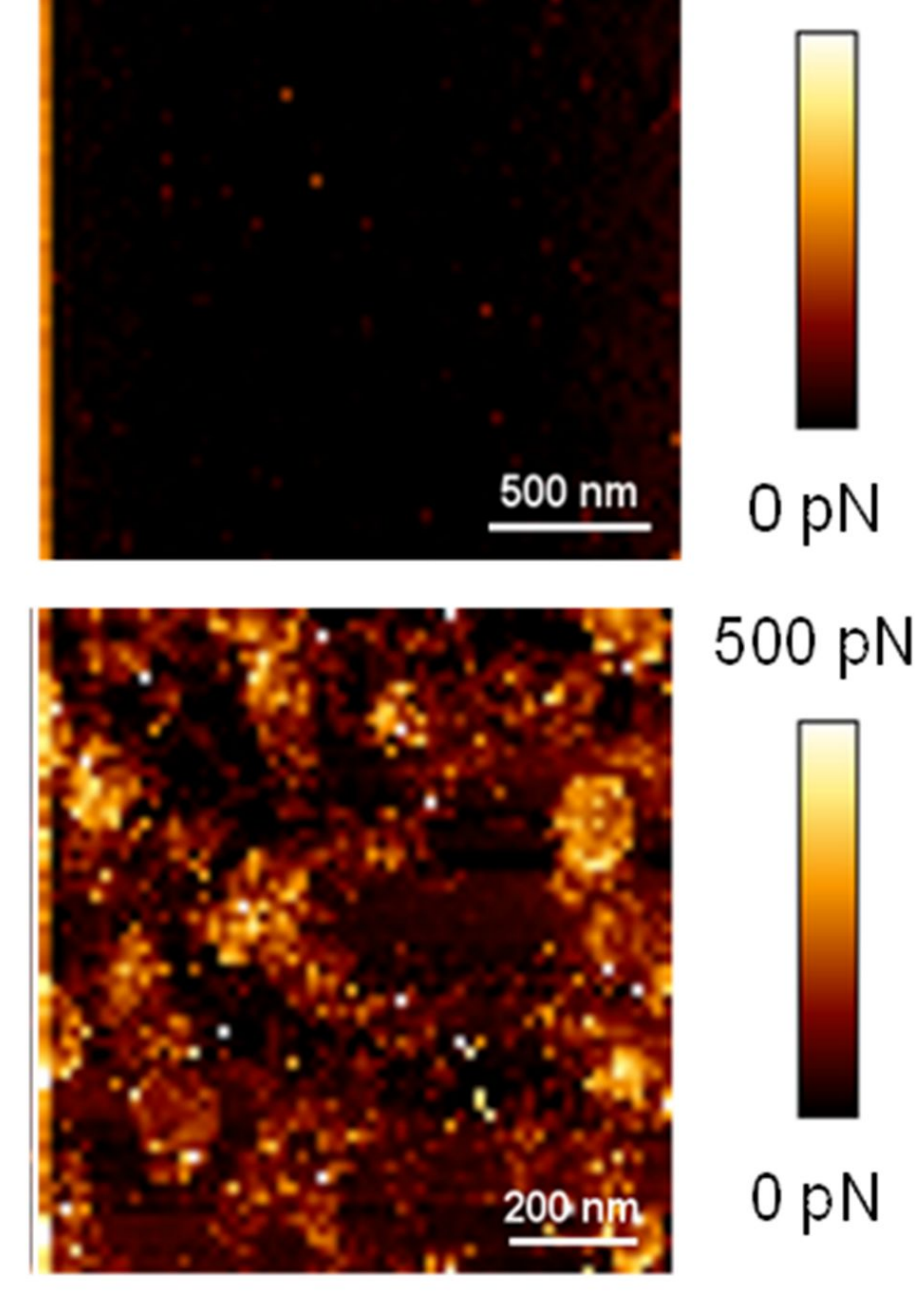


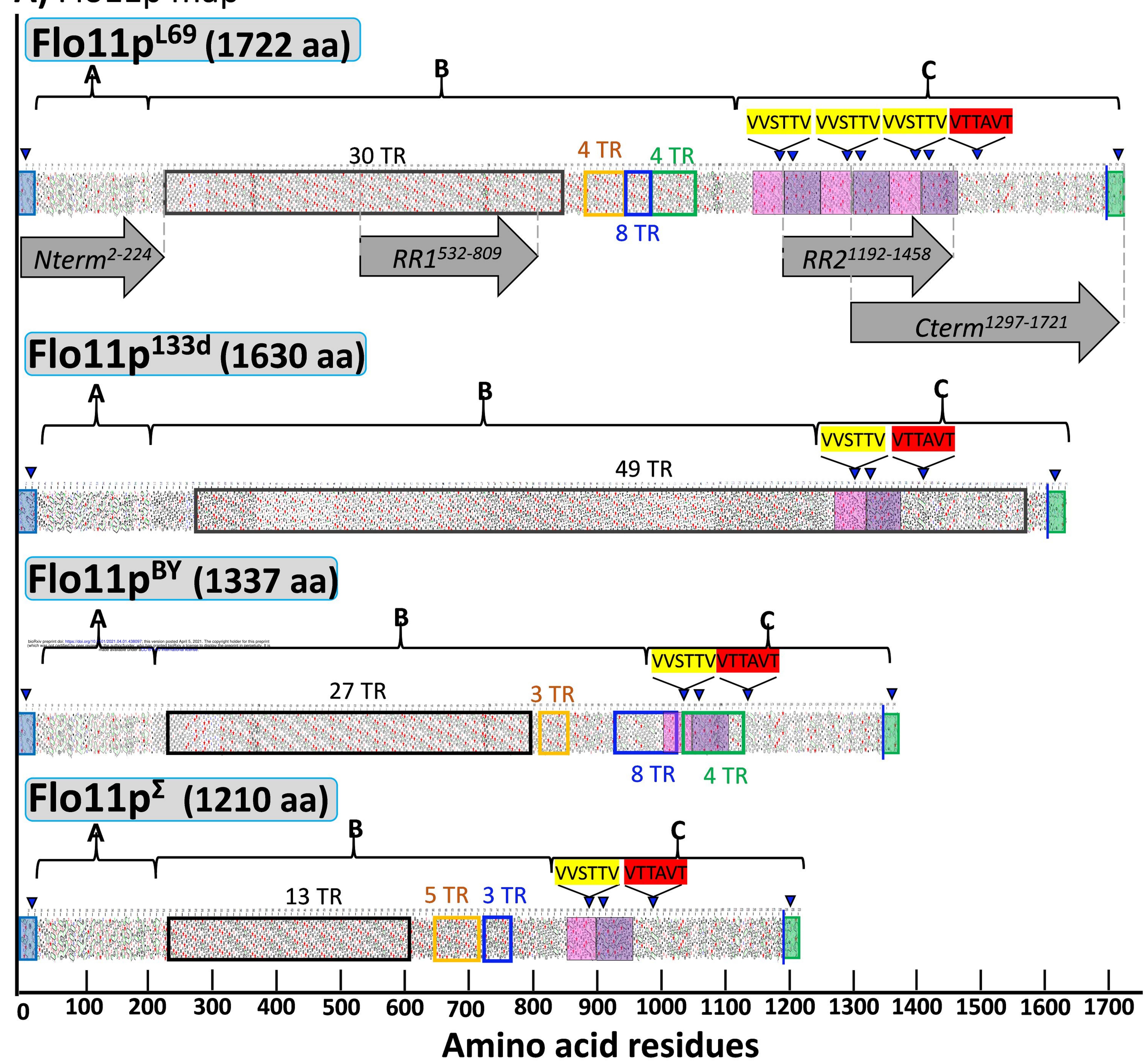

B) qRT-PCR of wild type and mutants of FLO11 gene

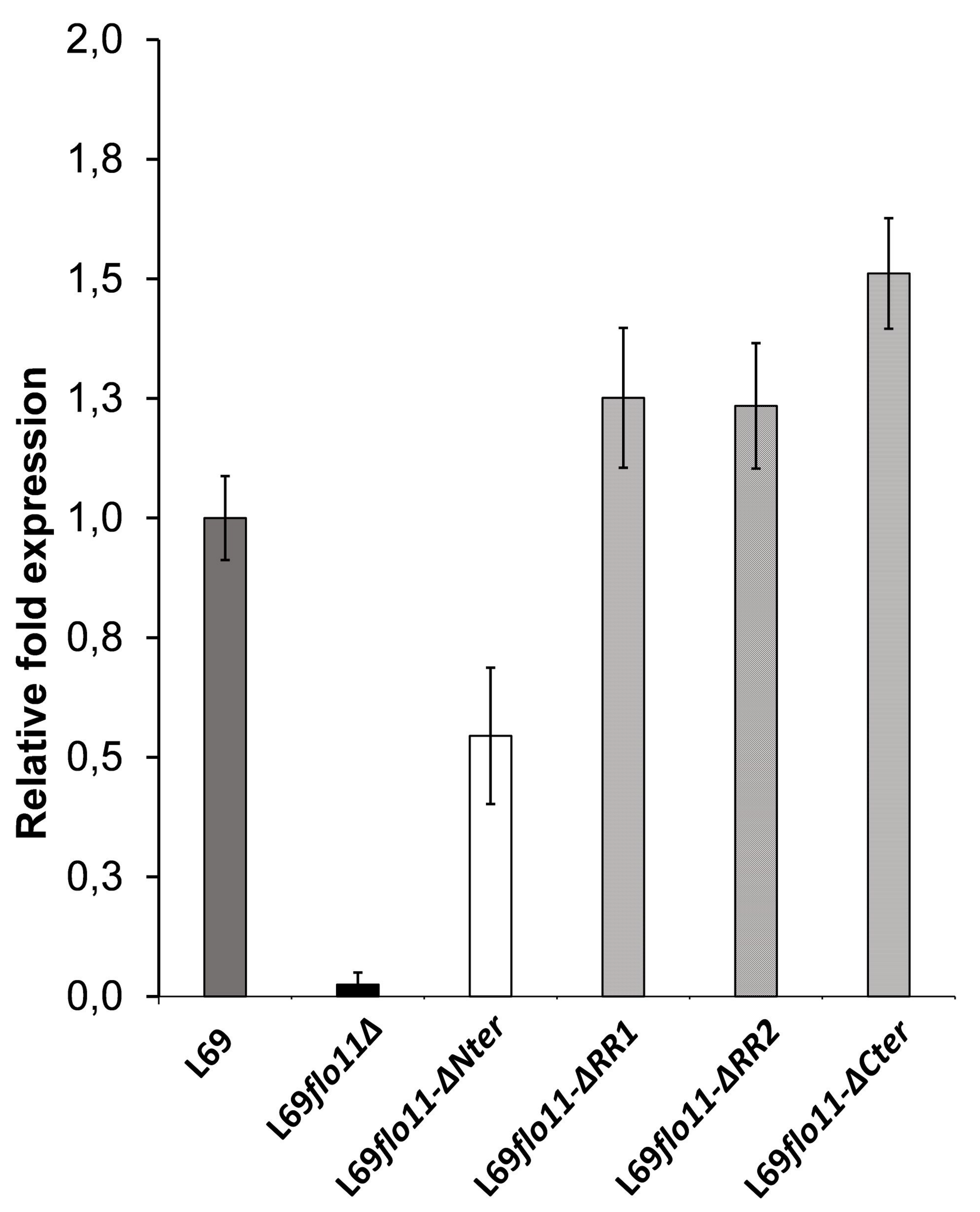


A

Height image

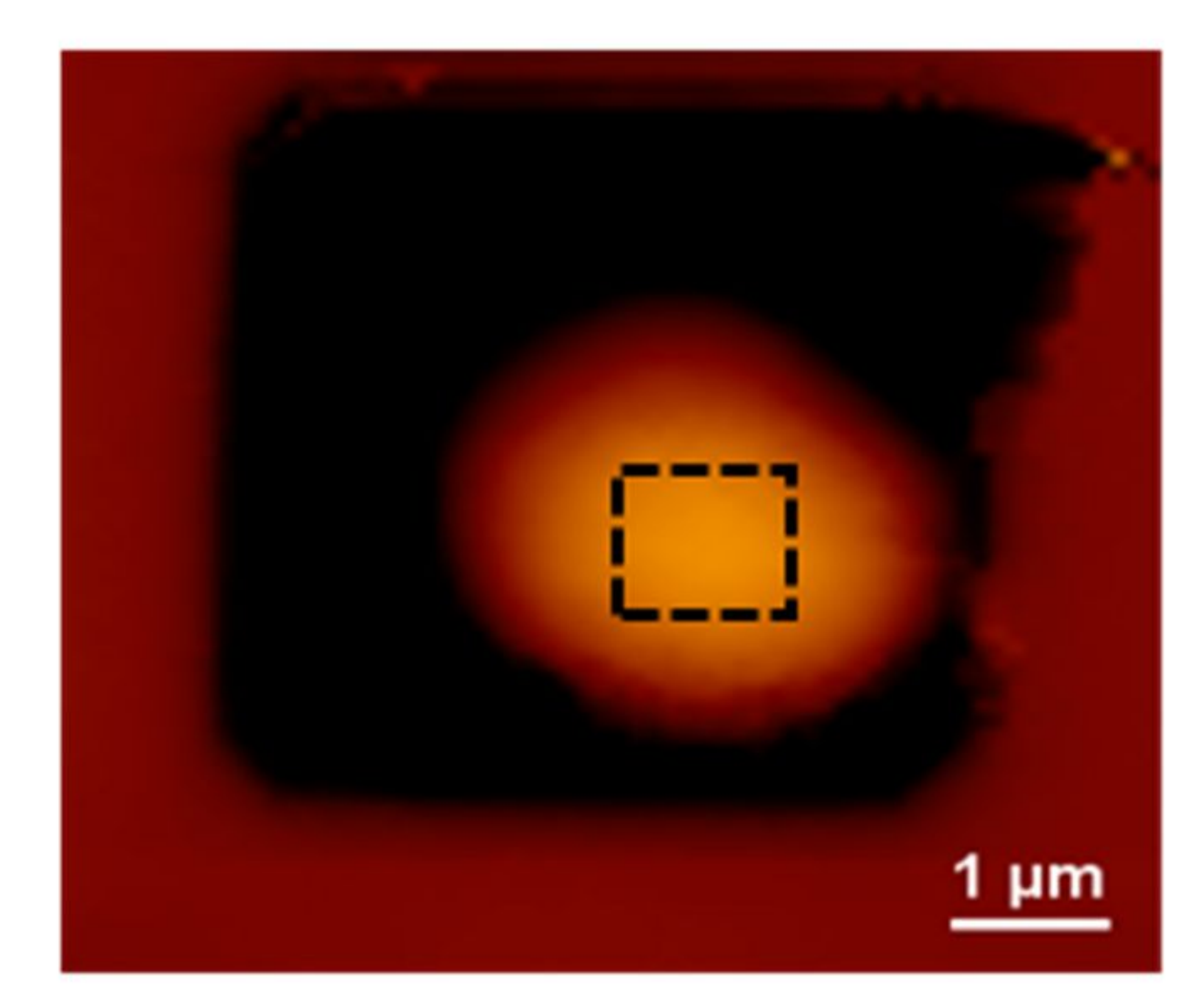

L69flo11-هNter

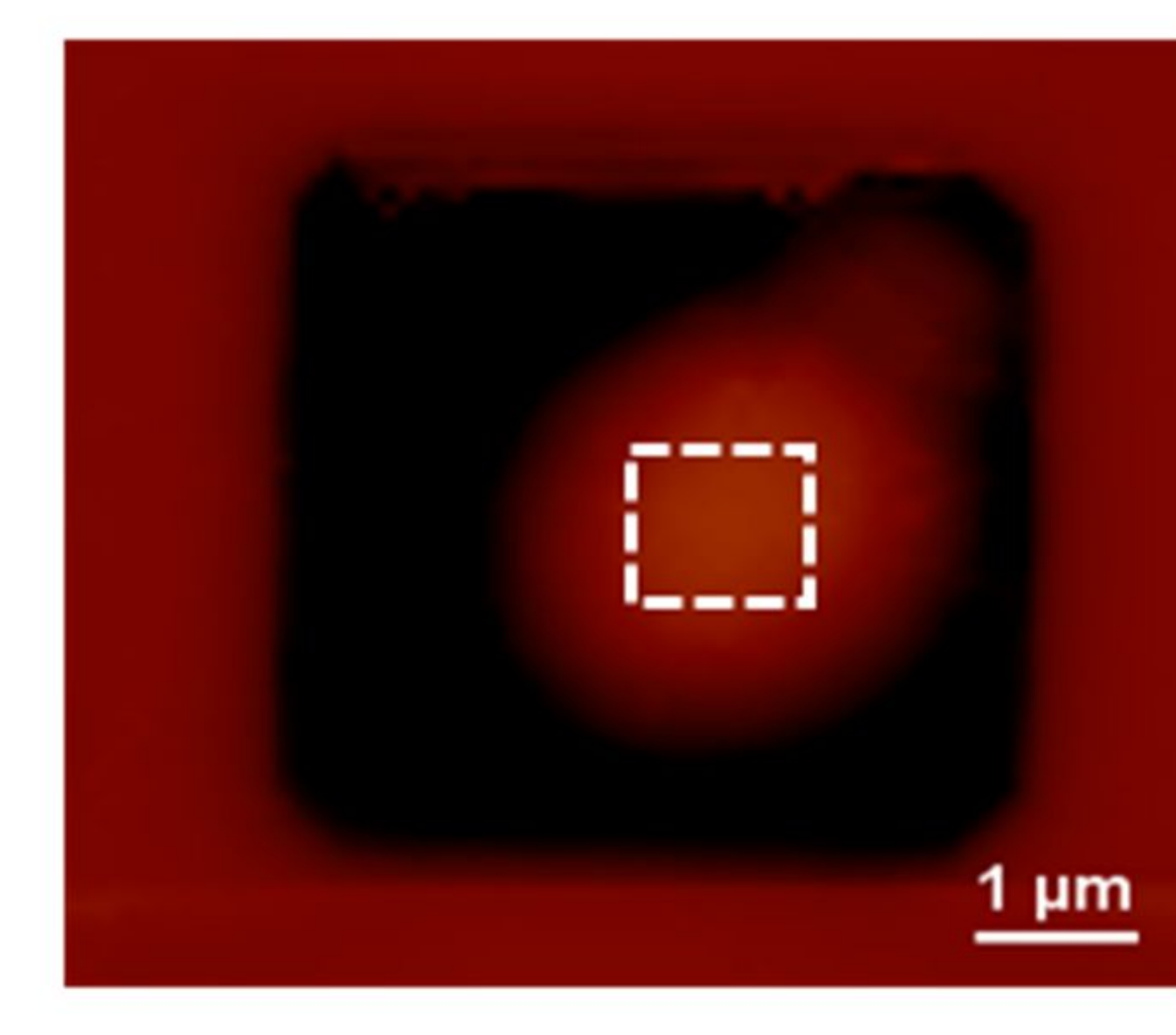

$0 \mu \mathrm{m}$

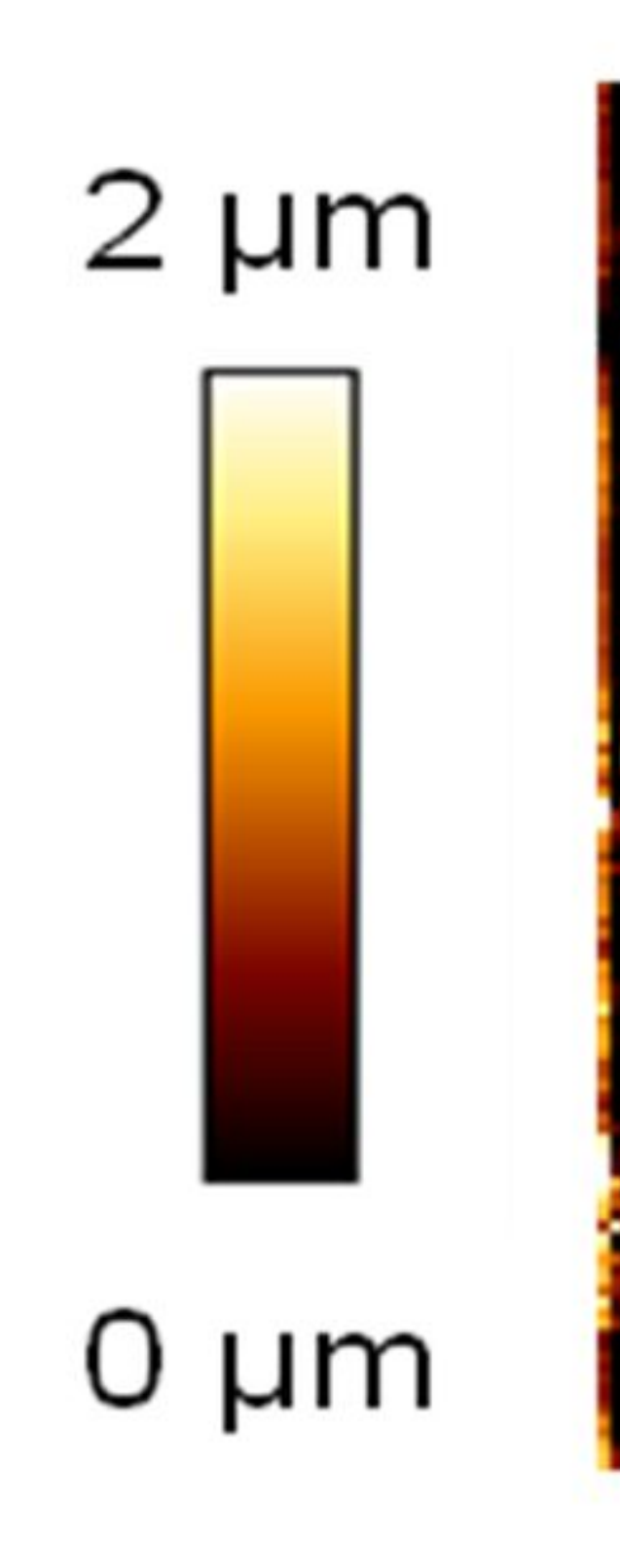

Adhesion image

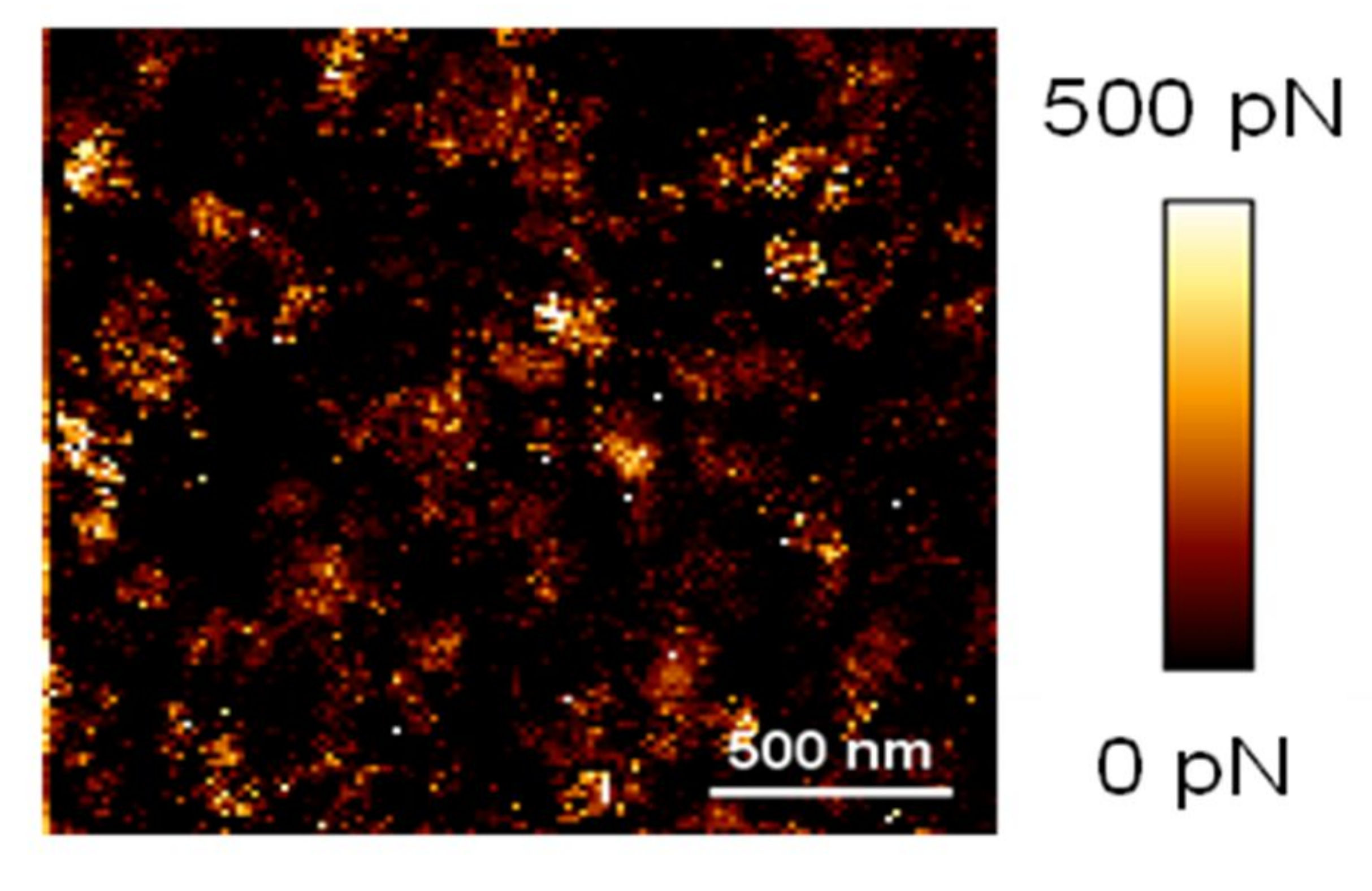

L69flo11-4Cter

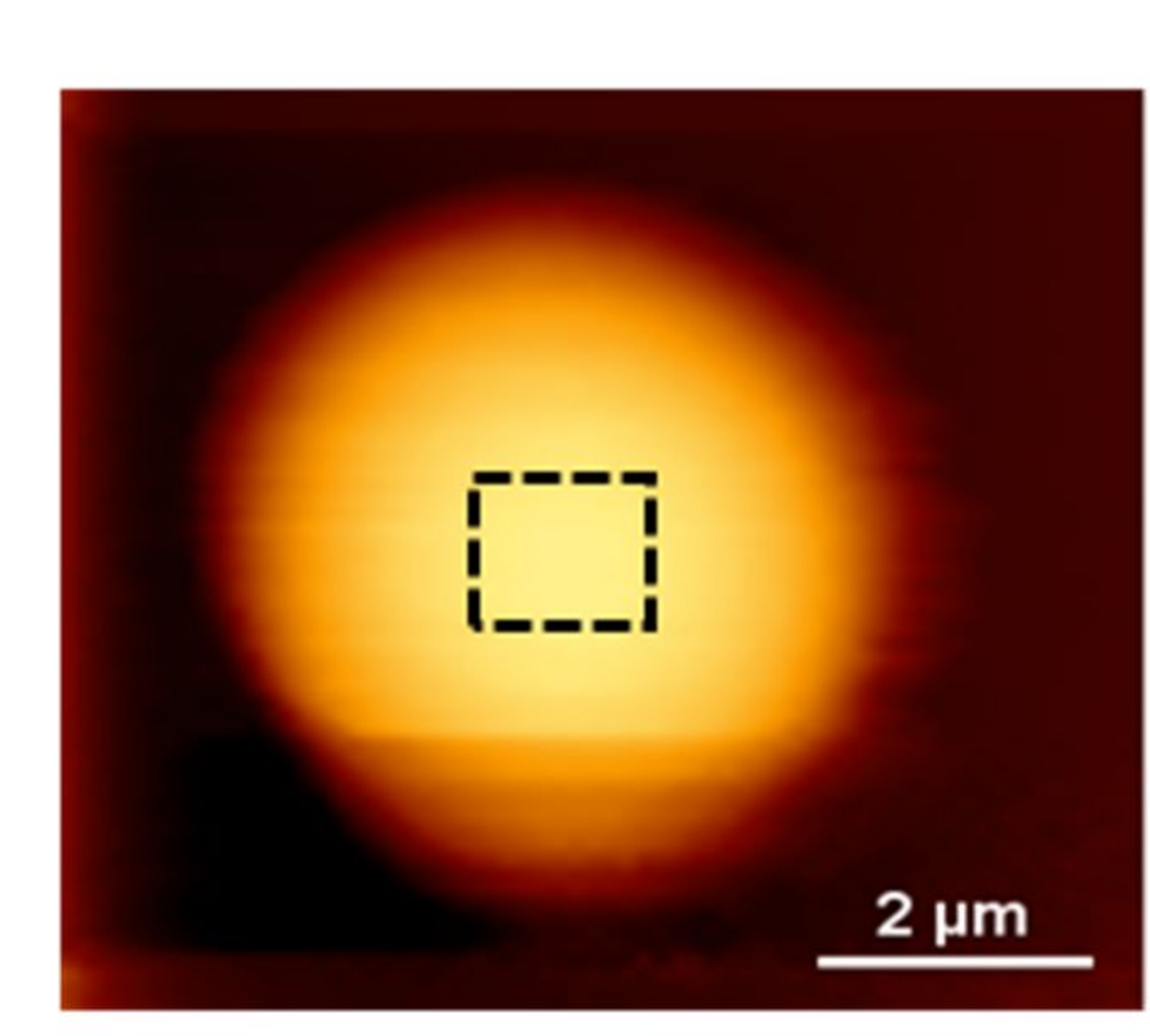

L69flo11- $\Delta R R 1$

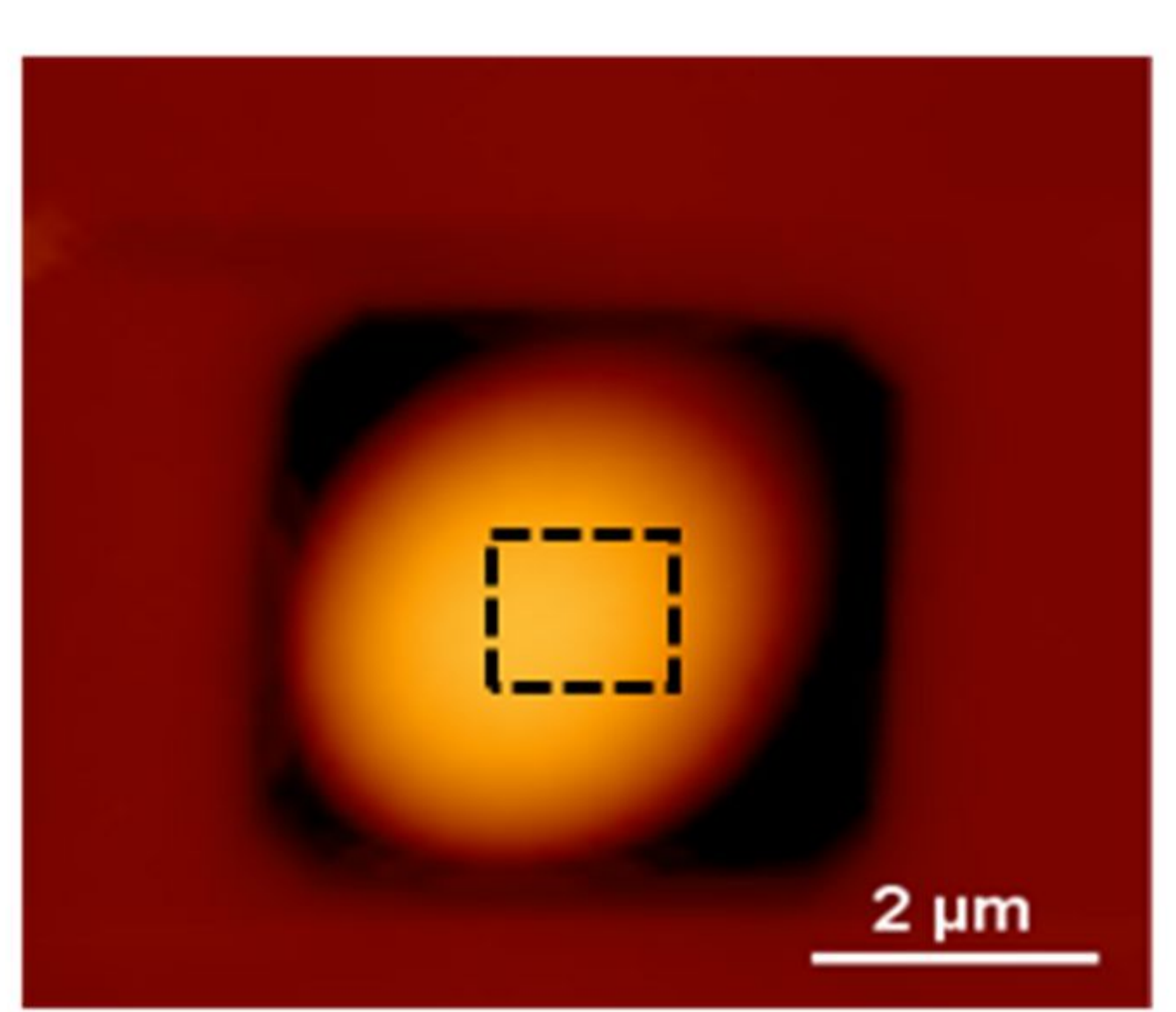

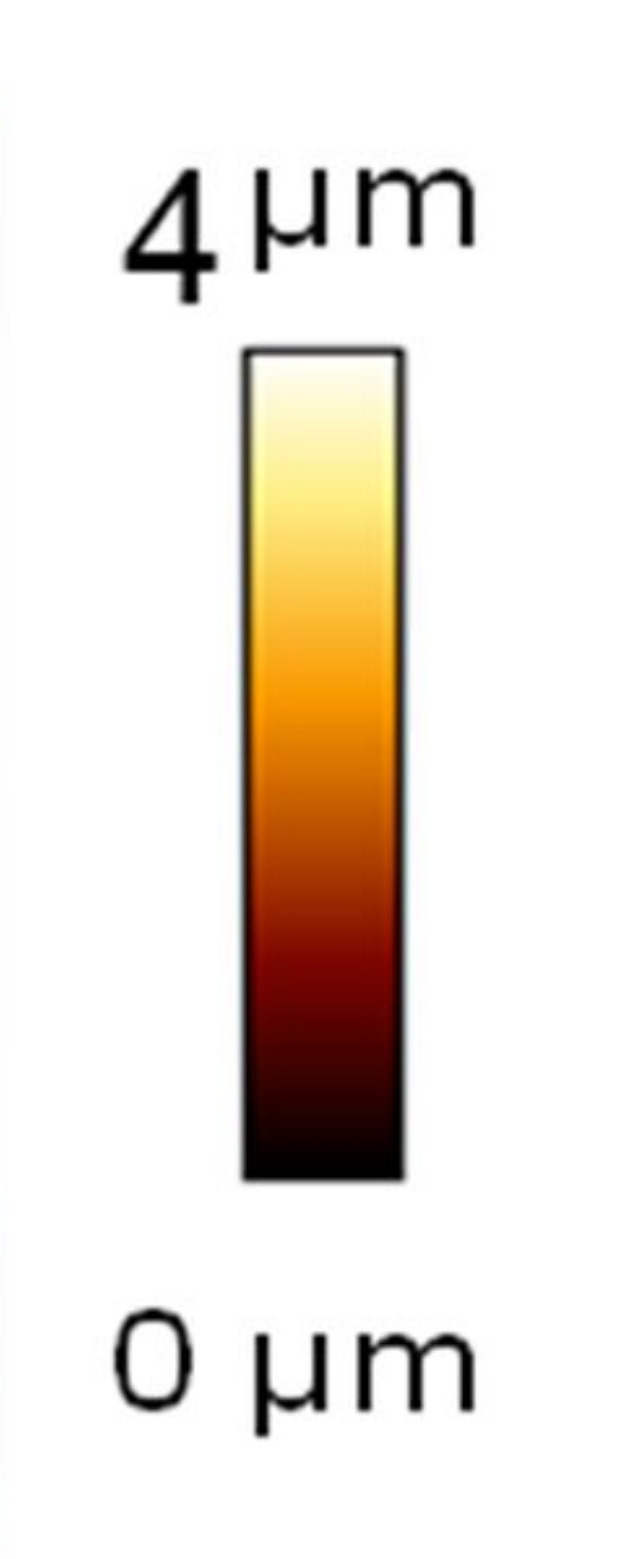

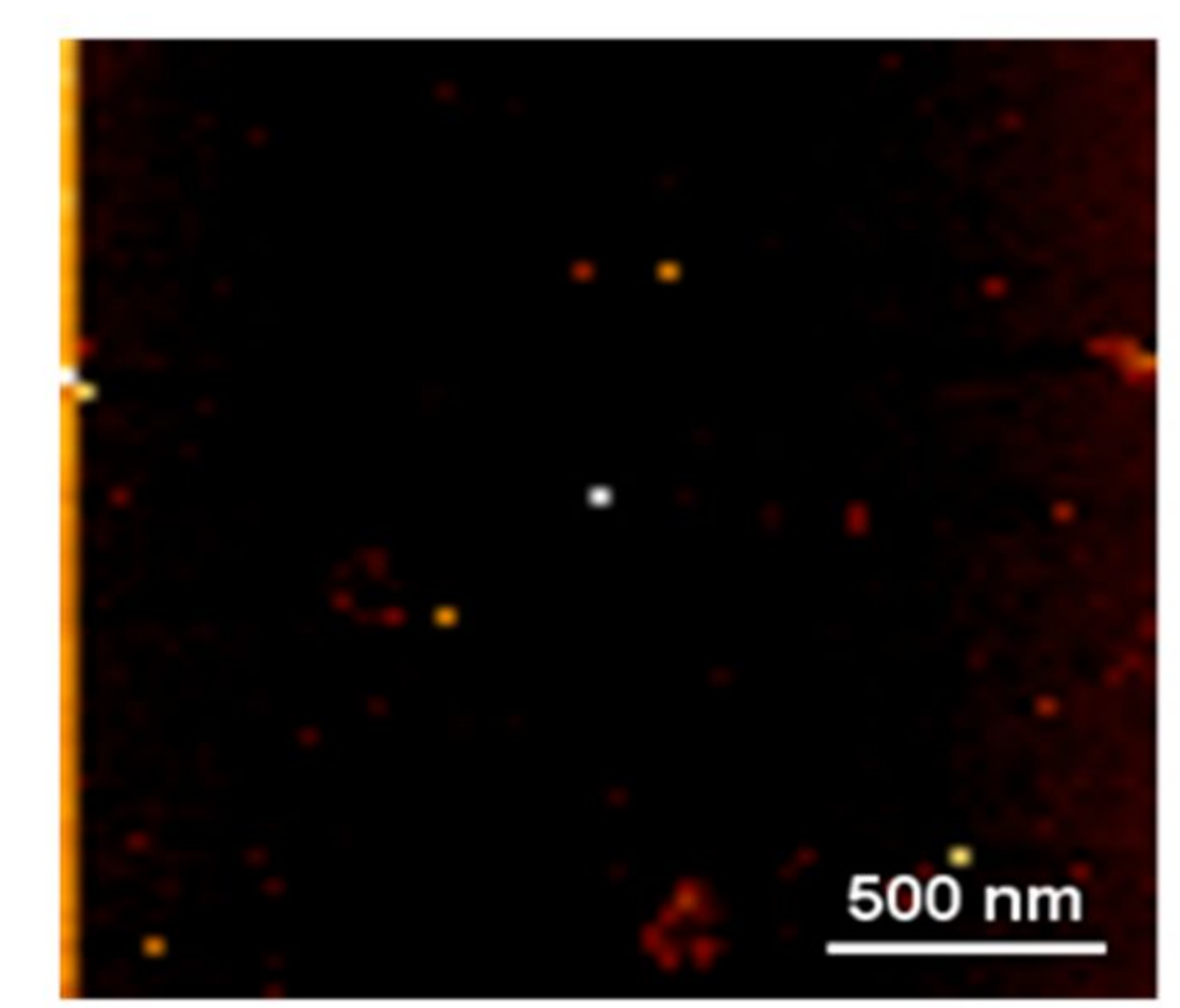

$500 \mathrm{pN}$
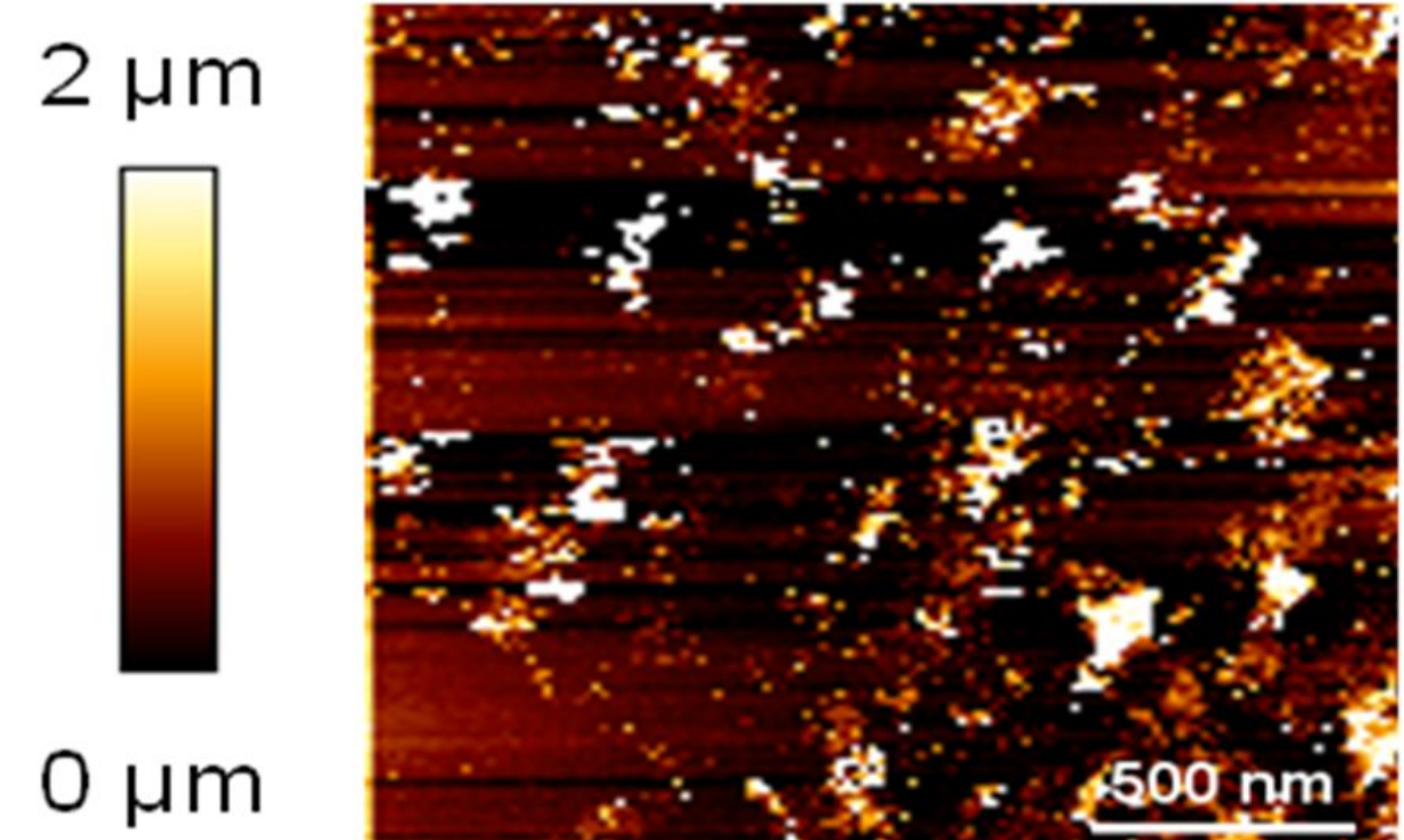

$500 \mathrm{pN}$
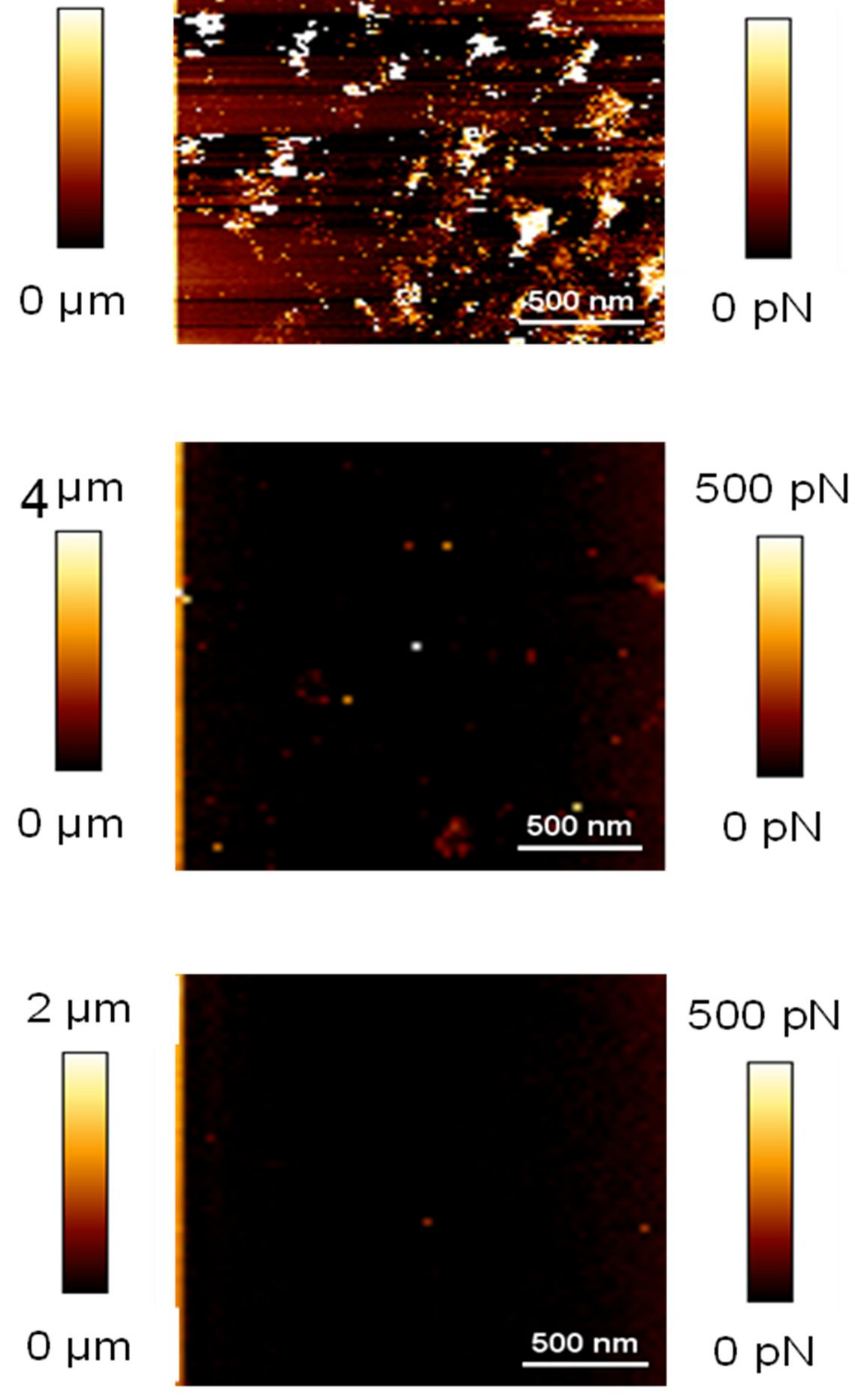

$500 \mathrm{pN}$
L69flo11- $\triangle R R 2$
B

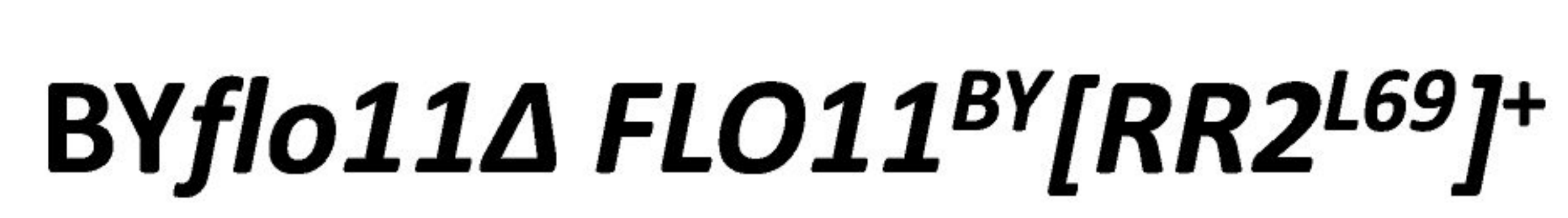

height image

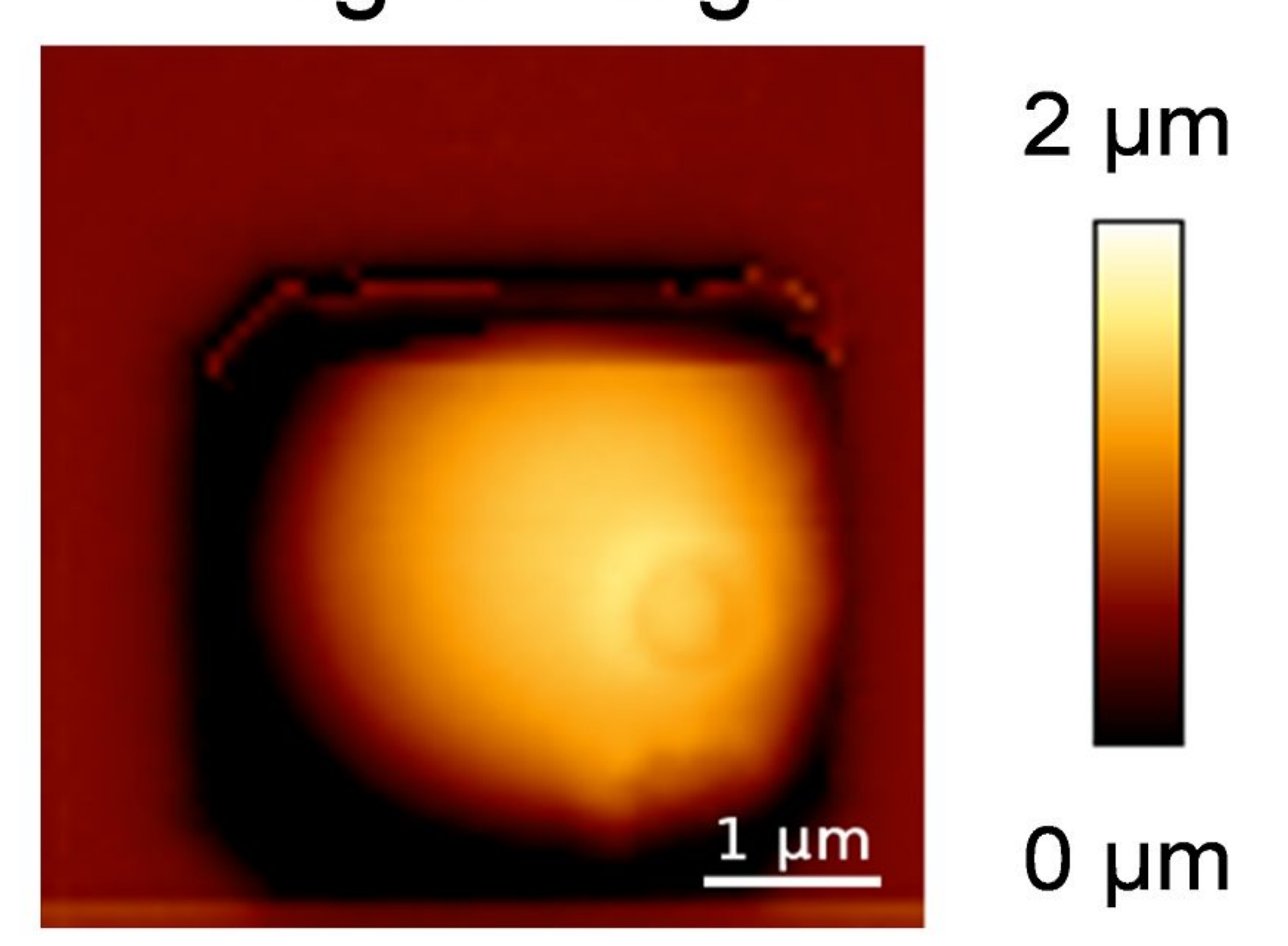

adhesion image

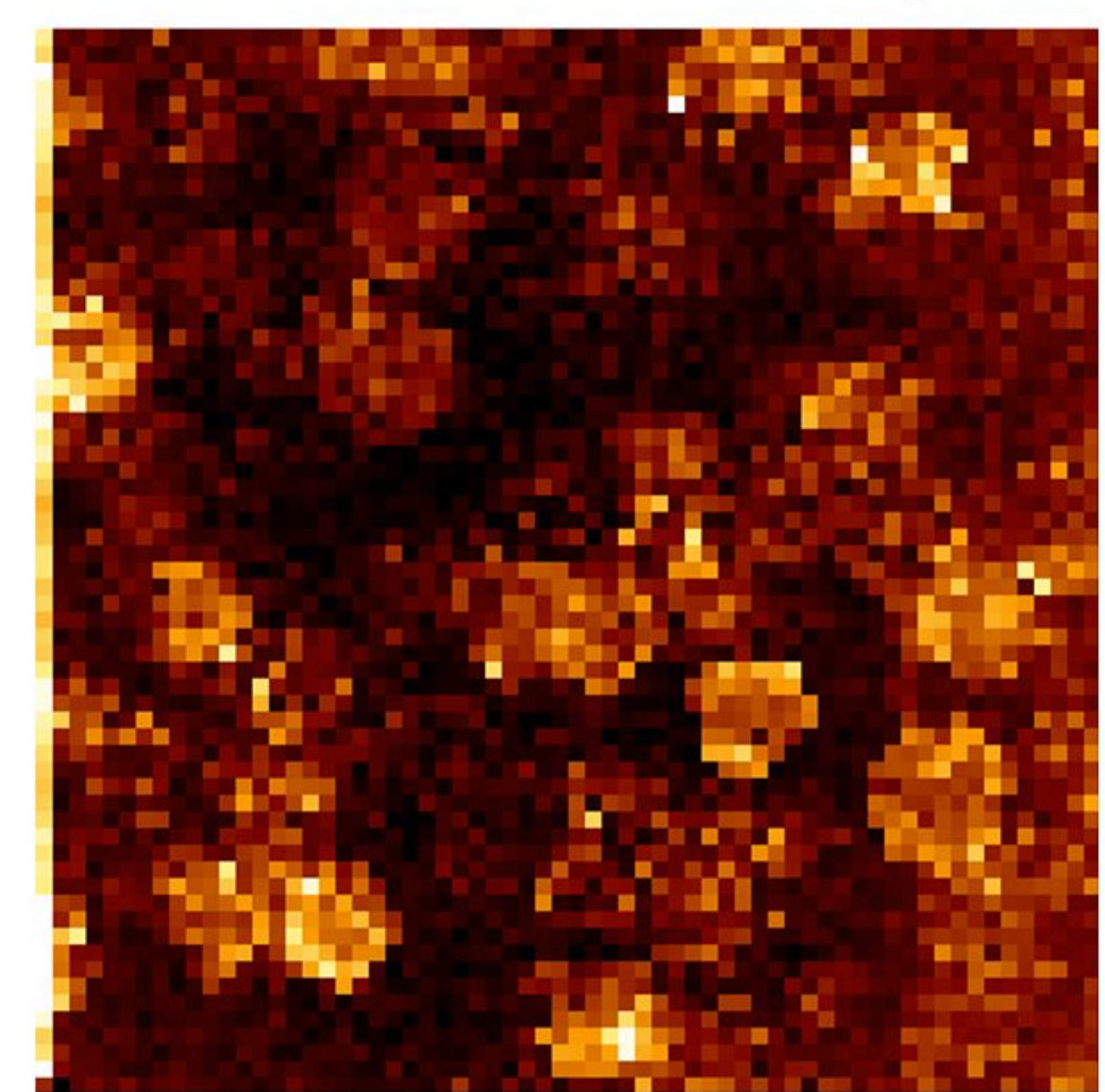

$500 \mathrm{pN}$
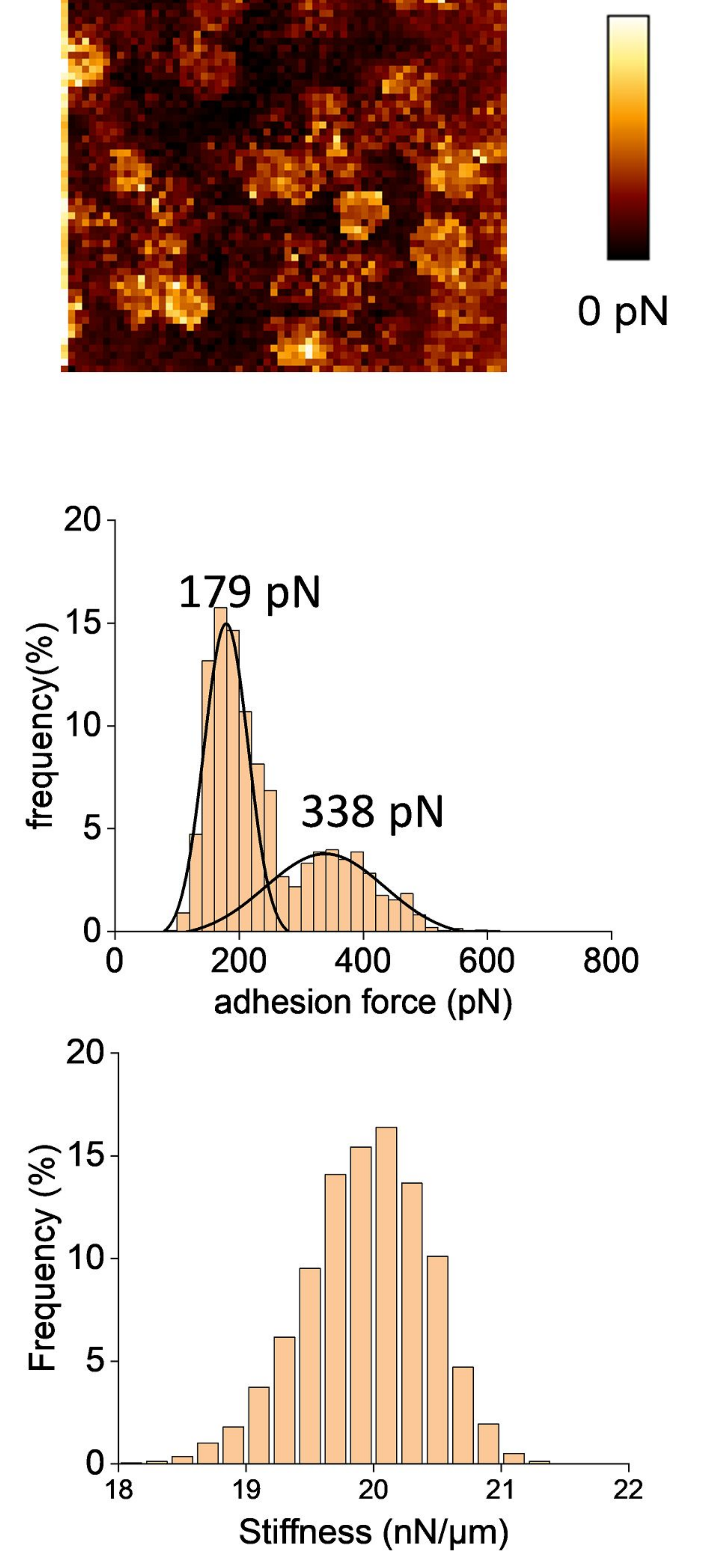

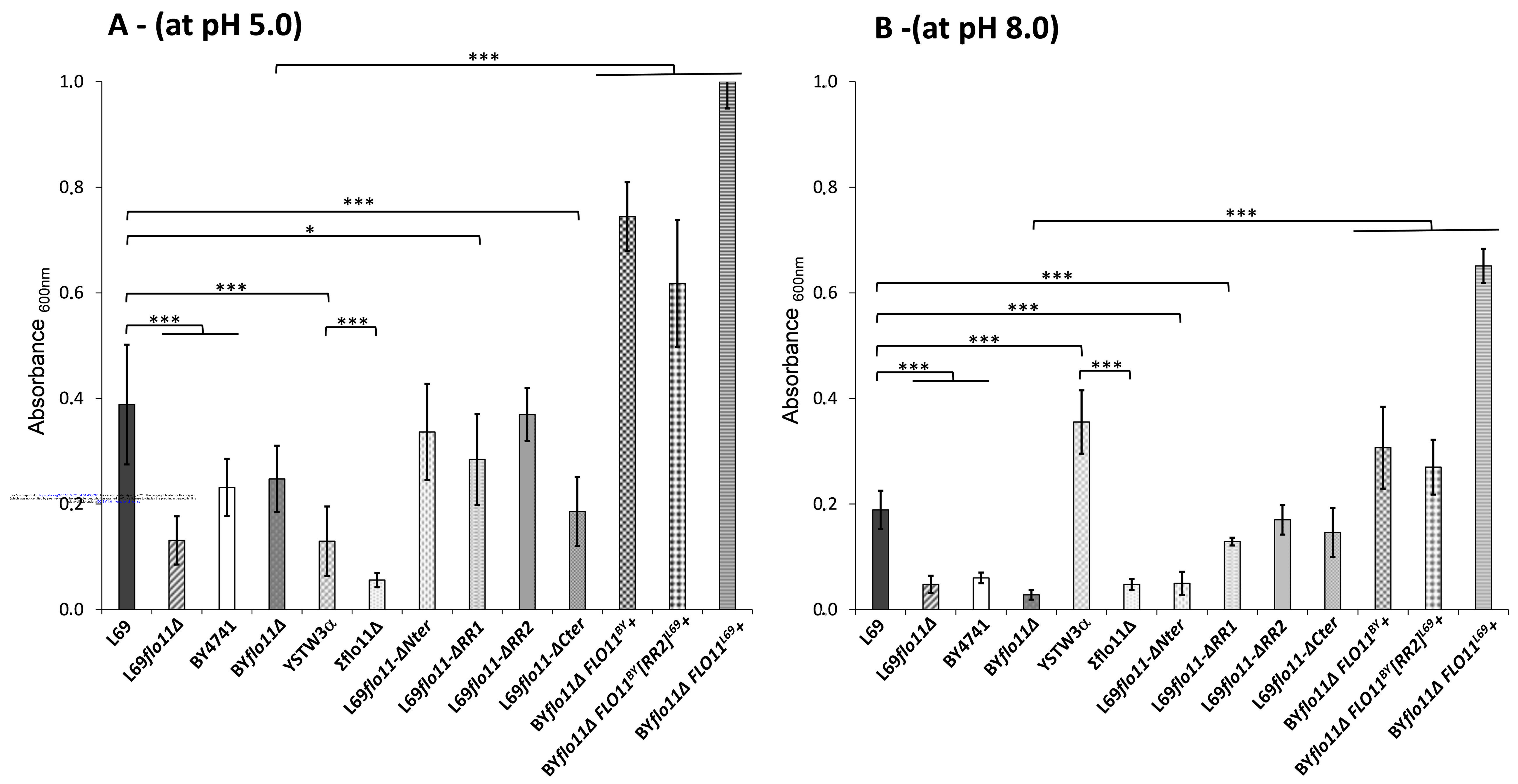


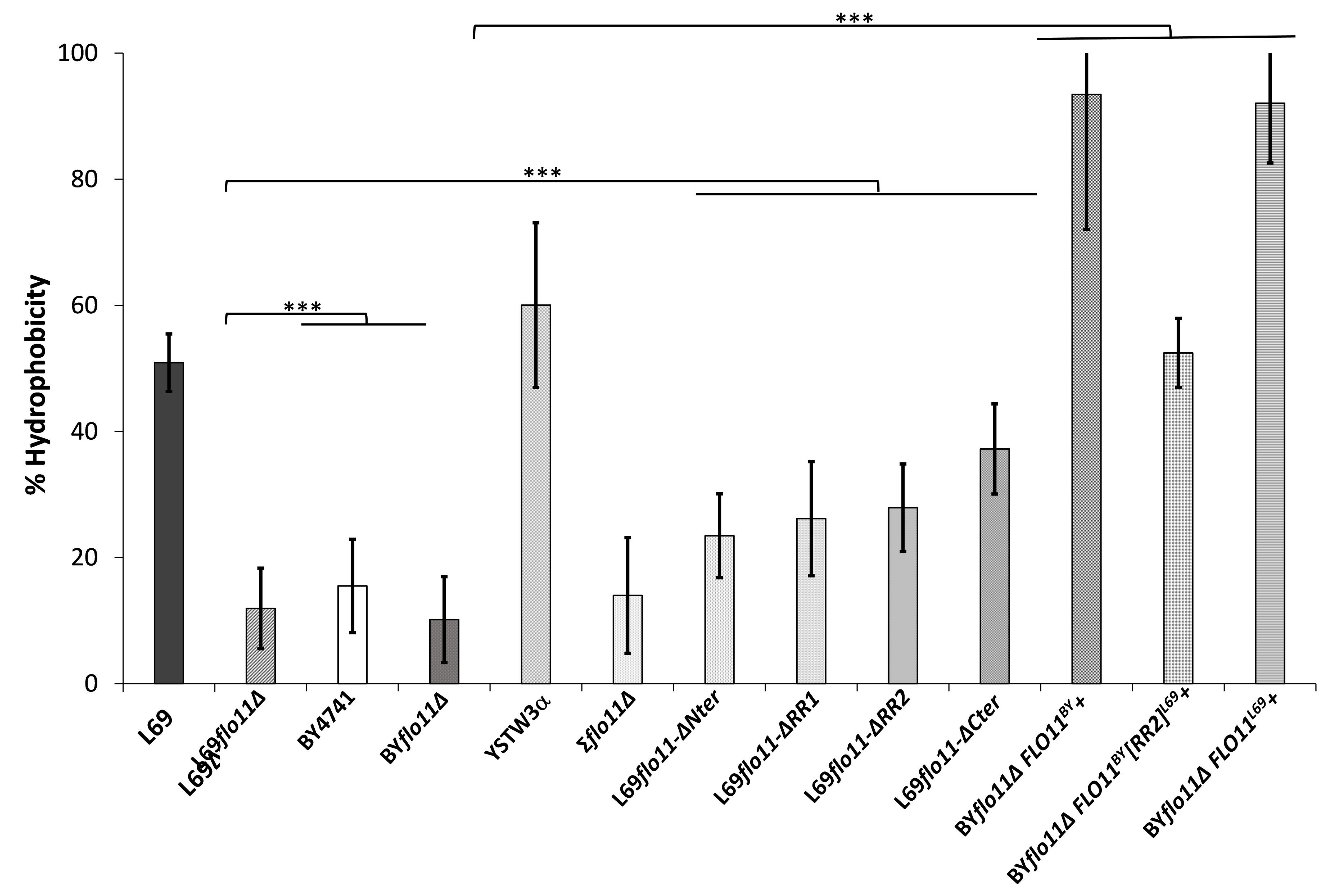


A

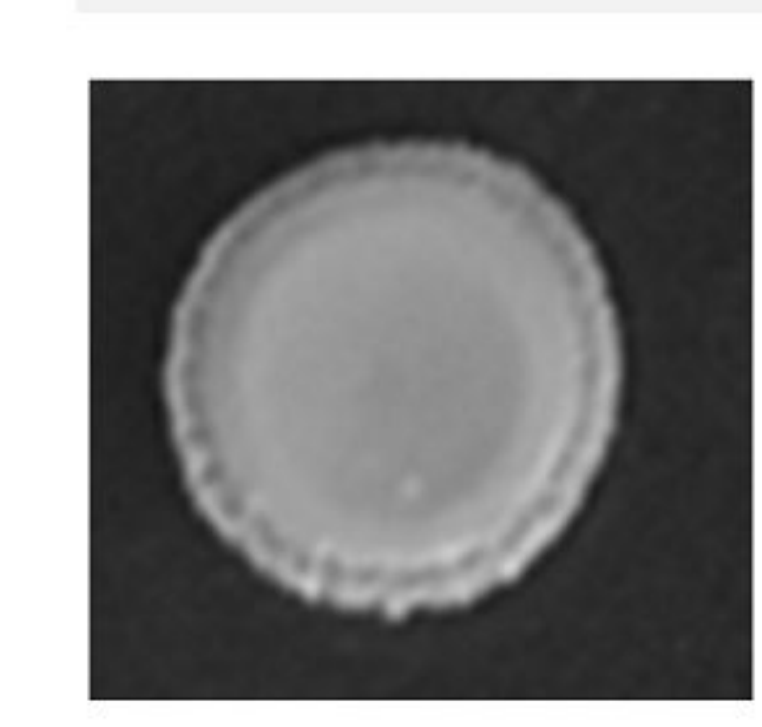

L69

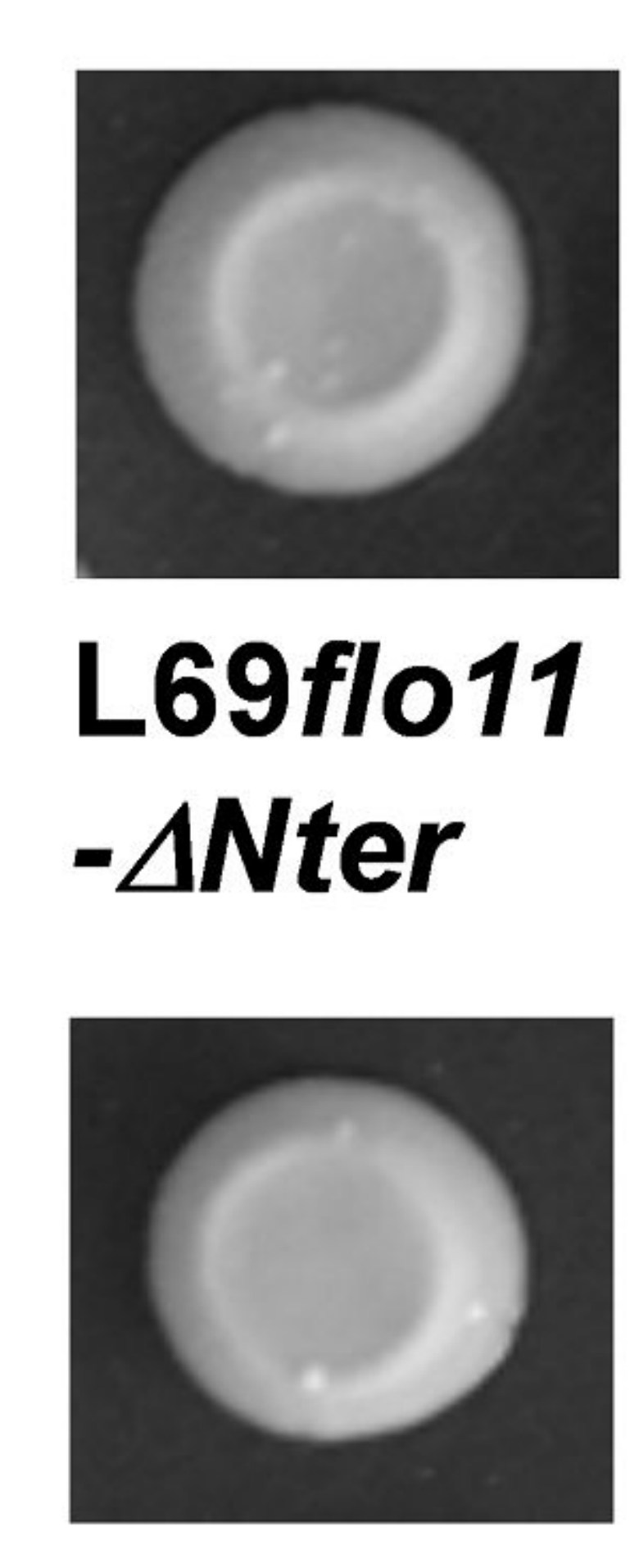

BYflo114

FLO11BY+

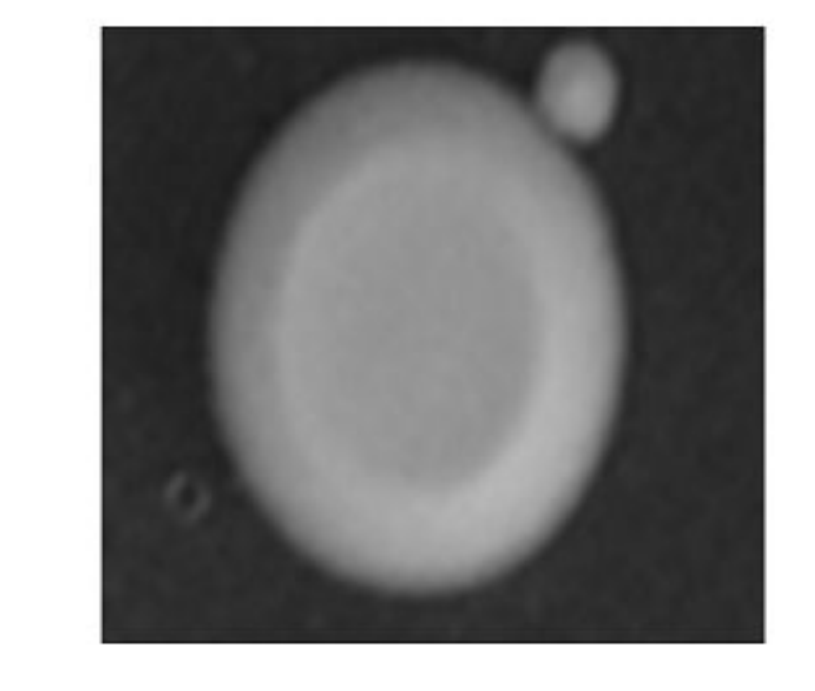

L69flo11A

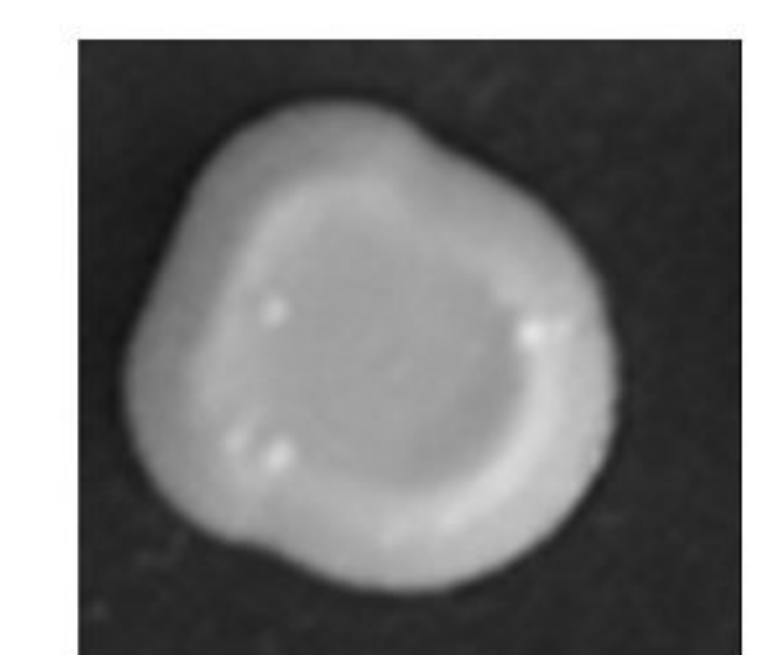

L69flo11 $-\Delta R R 1$

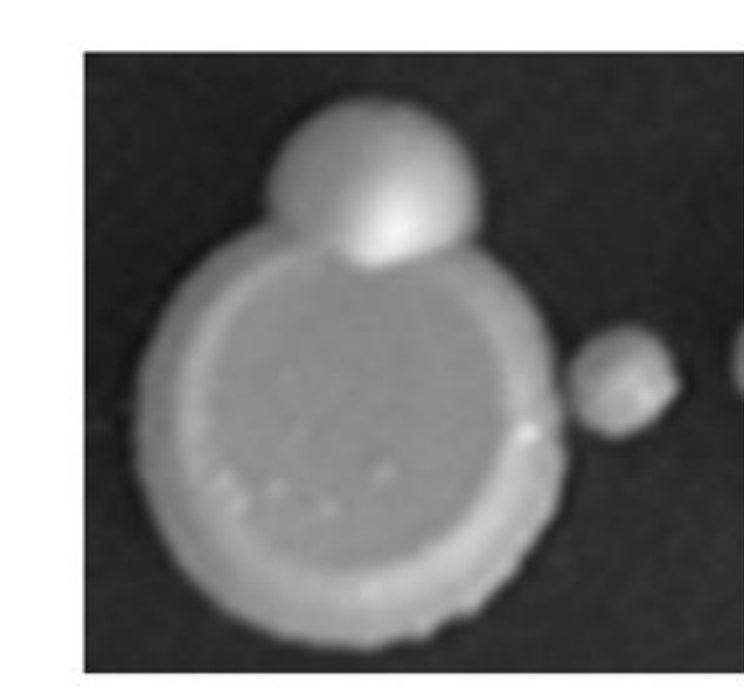

BYflo11A FL011 ${ }^{L 69+}$
YPD / Before wash

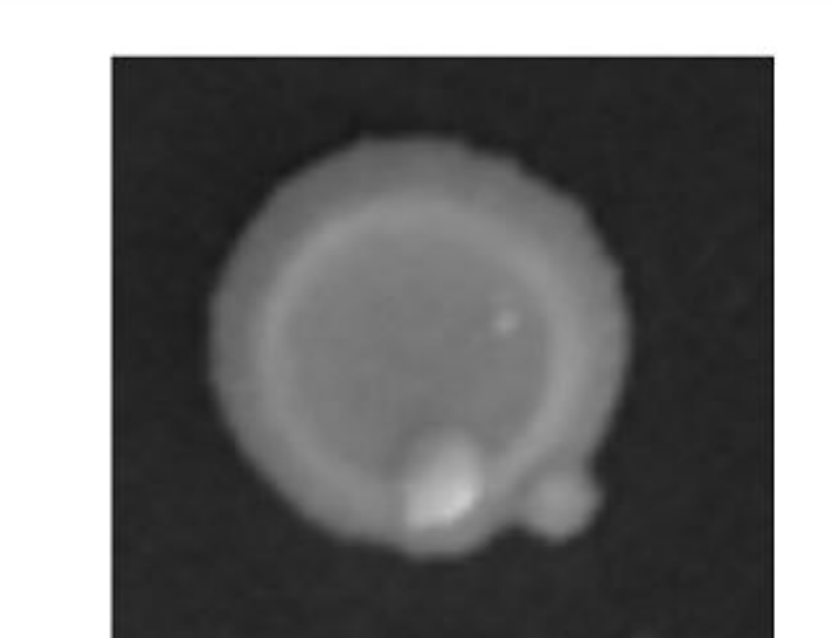

BY4741

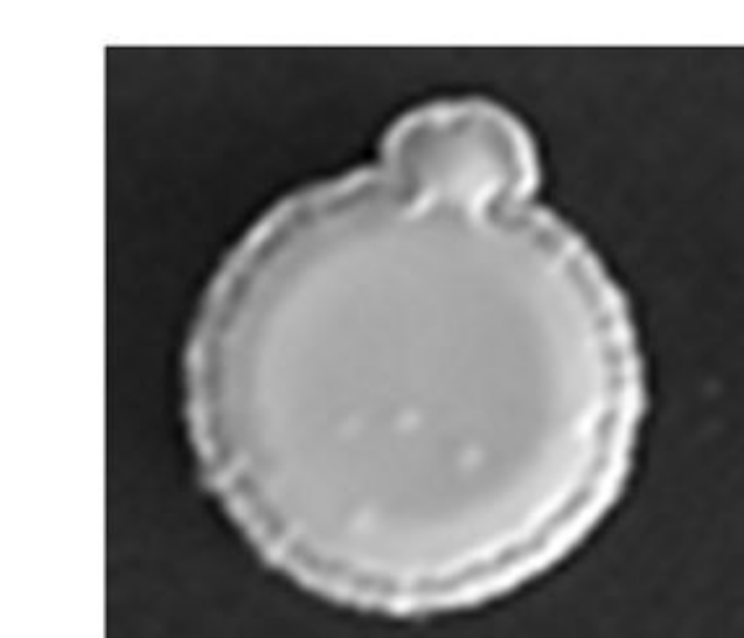

L69flo11

$-\Delta R R 2$

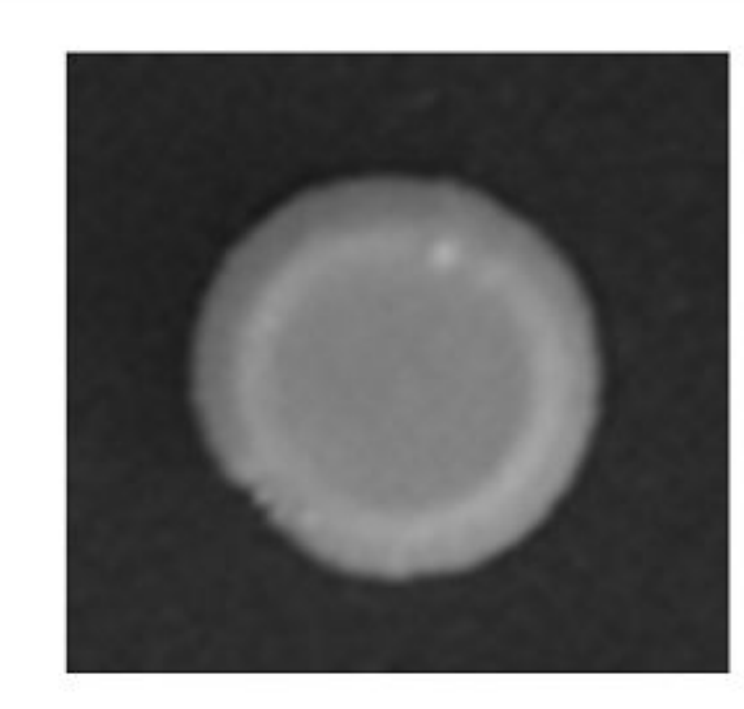

BYflo114

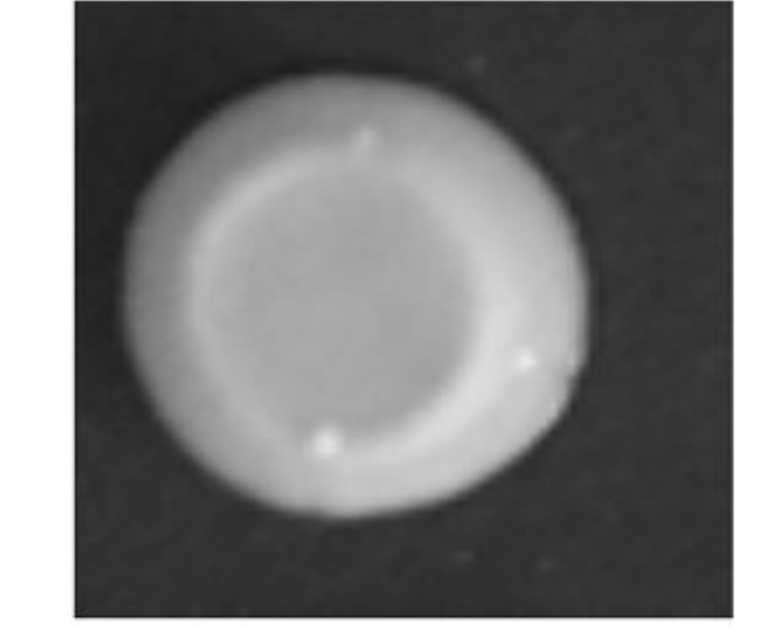

L69flo11

- $\Delta$ Cter

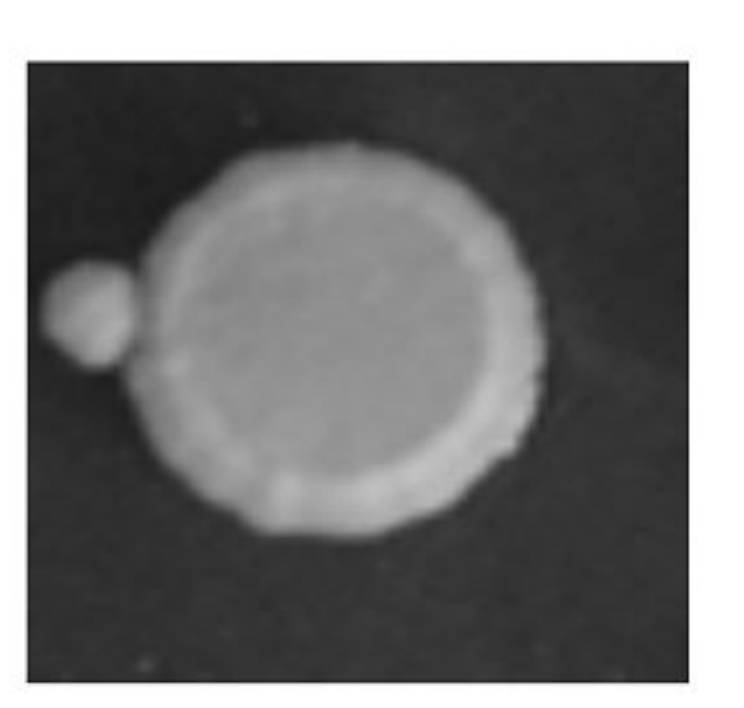

BYflo11A

FLO11 $\left.^{B Y}{ }_{-[R R 2}\right]^{L 69+}$

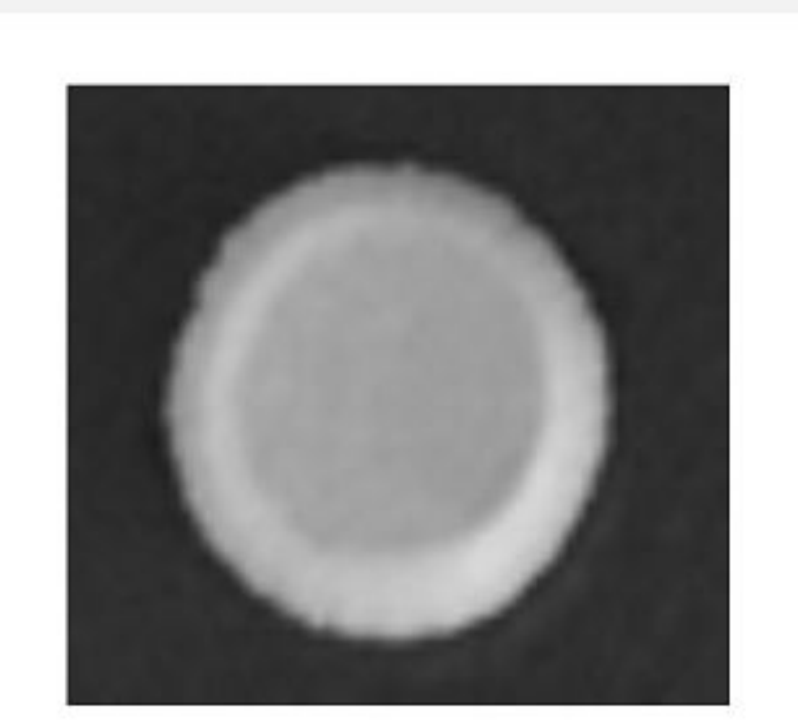

YSWT3 $\alpha$

इflo114
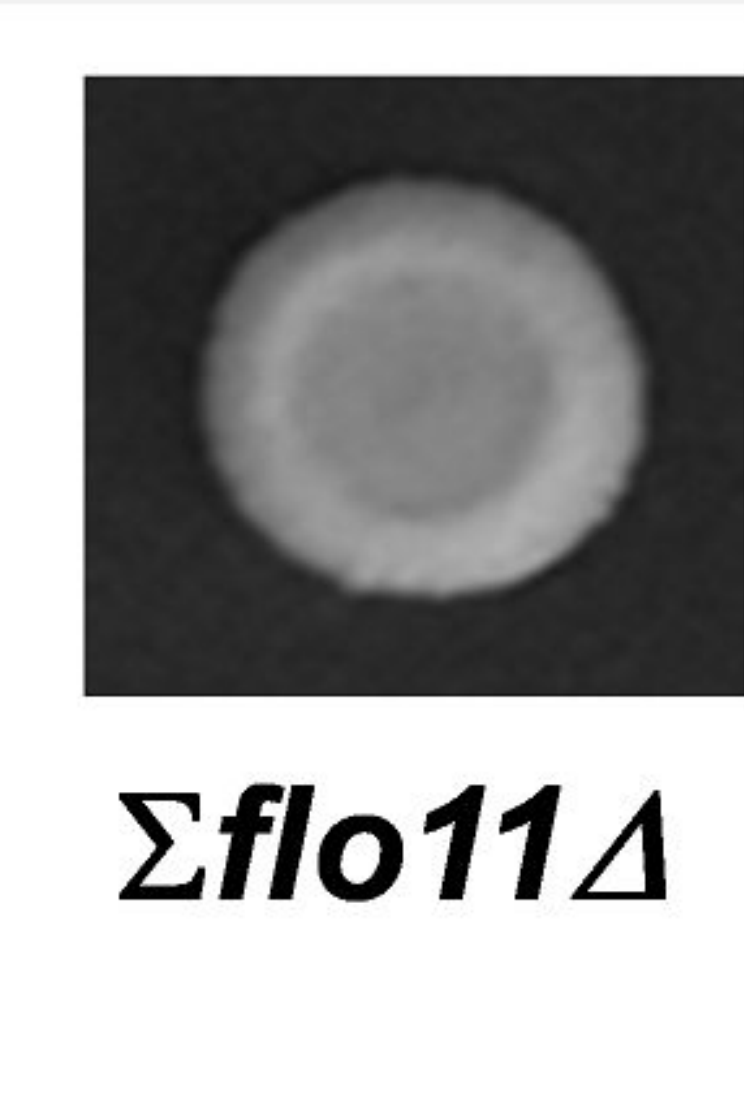

B

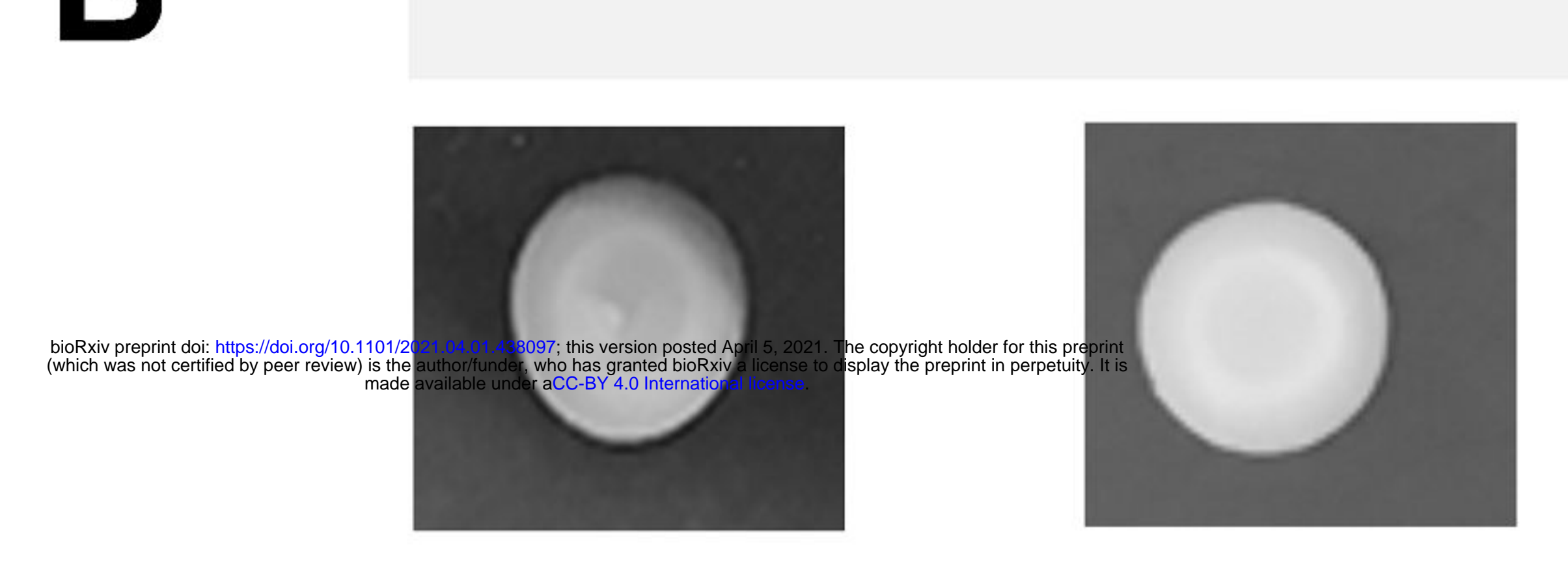

L69

L69flo11A

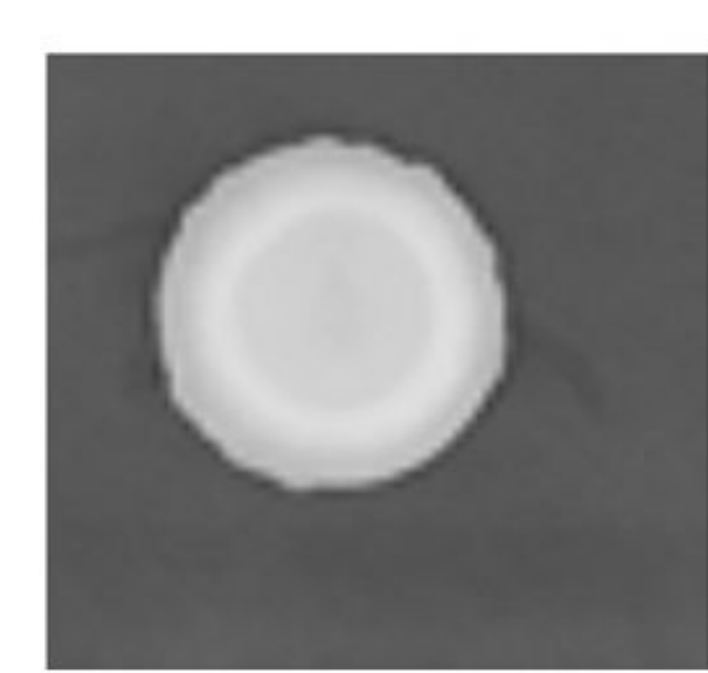

L69flo11 -ANter

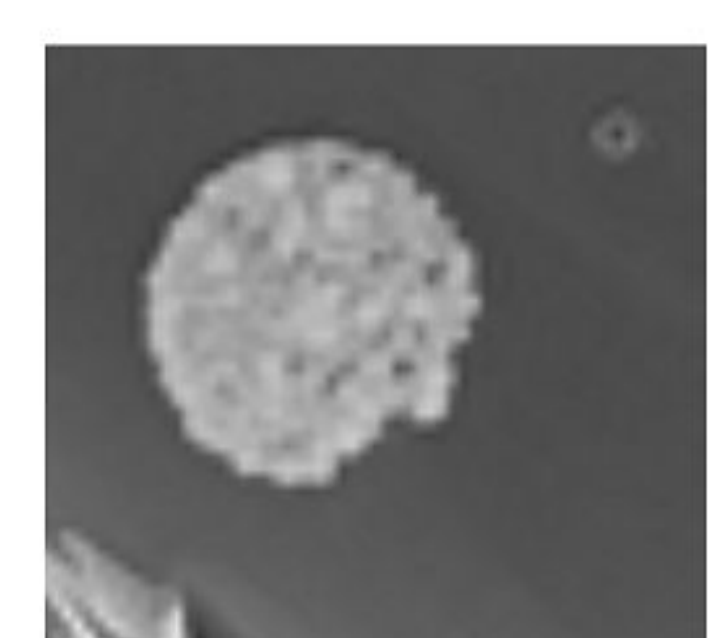

BYflo11A FLO11BY+

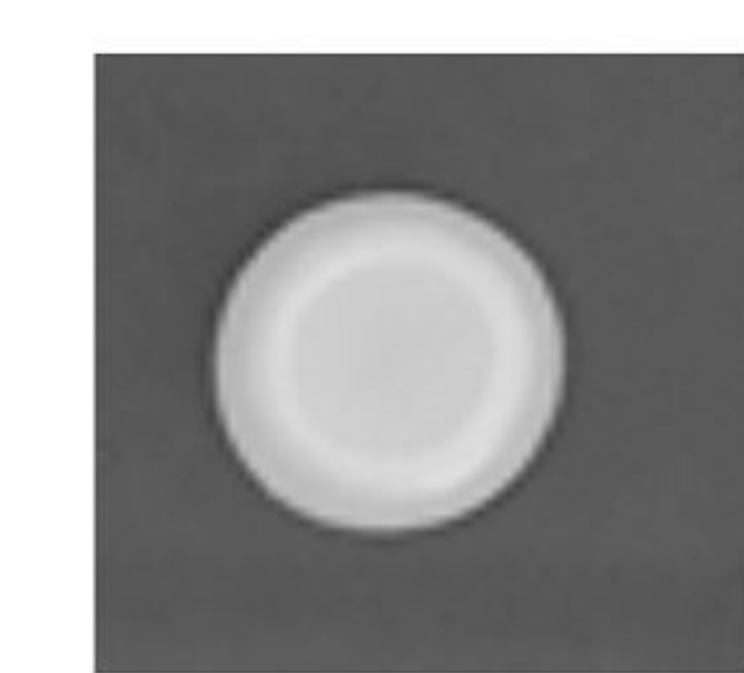

L69flo11 - $\Delta R R 1$

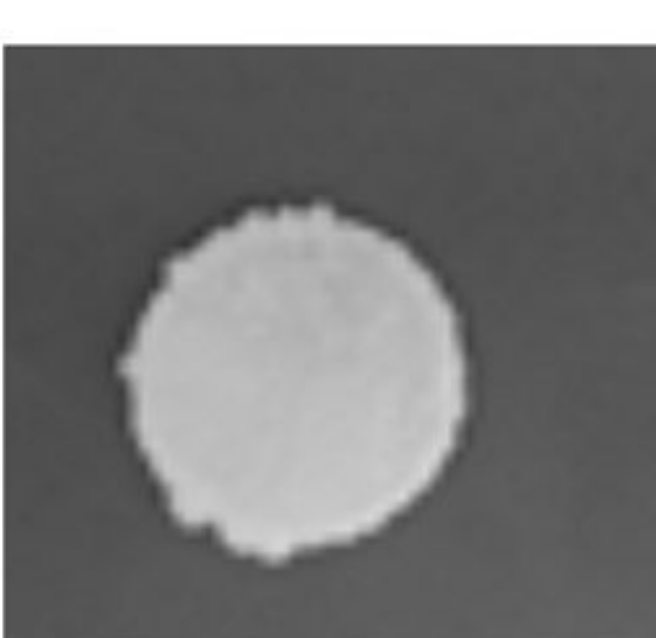

BYflo11A FLO11L69+
YNGal / Before wash

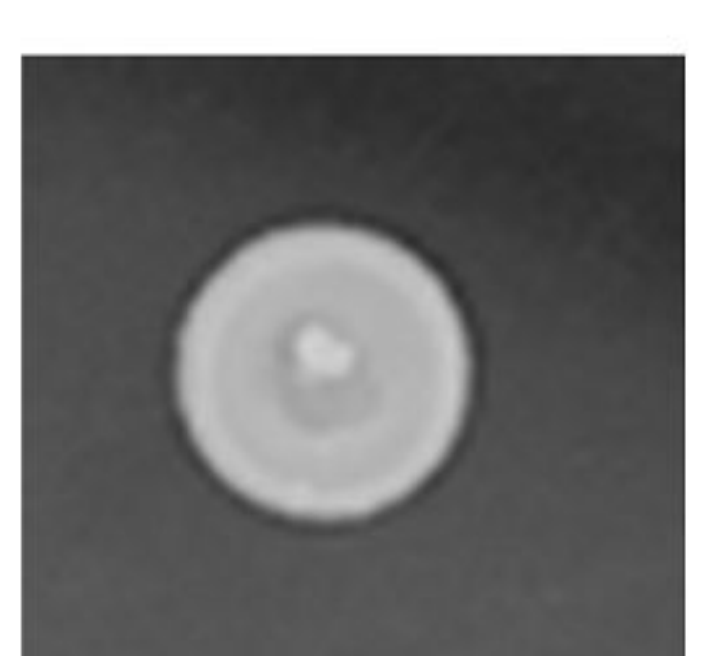

BY4741

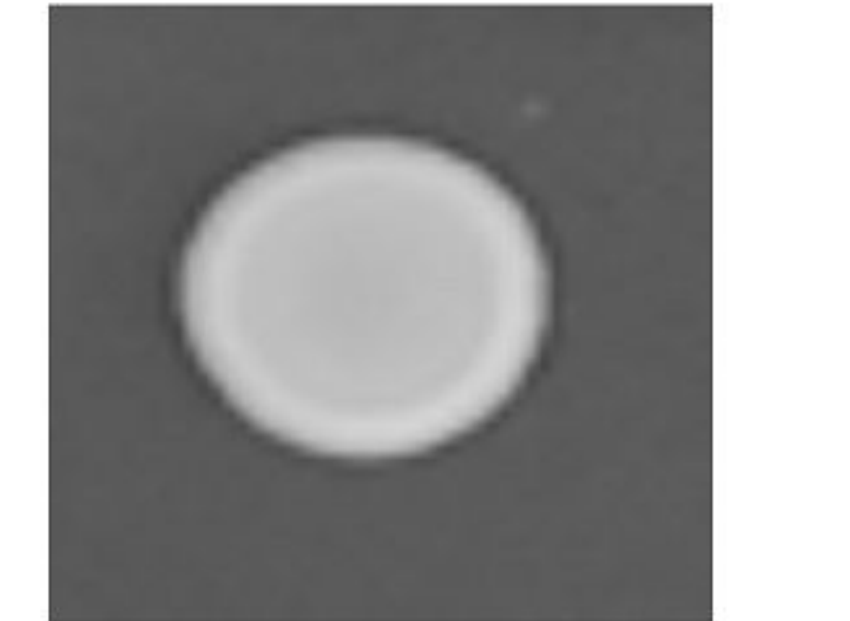

BYflo114

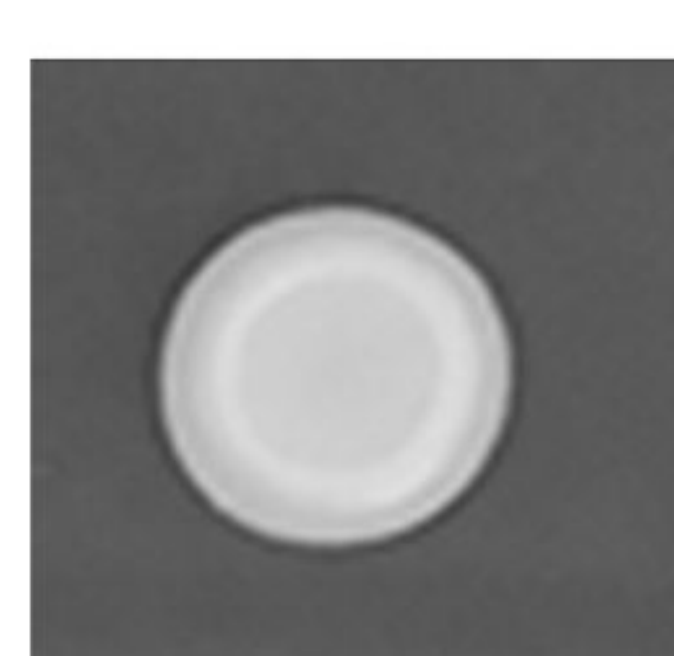

L69flo11 - $\Delta R R 2$
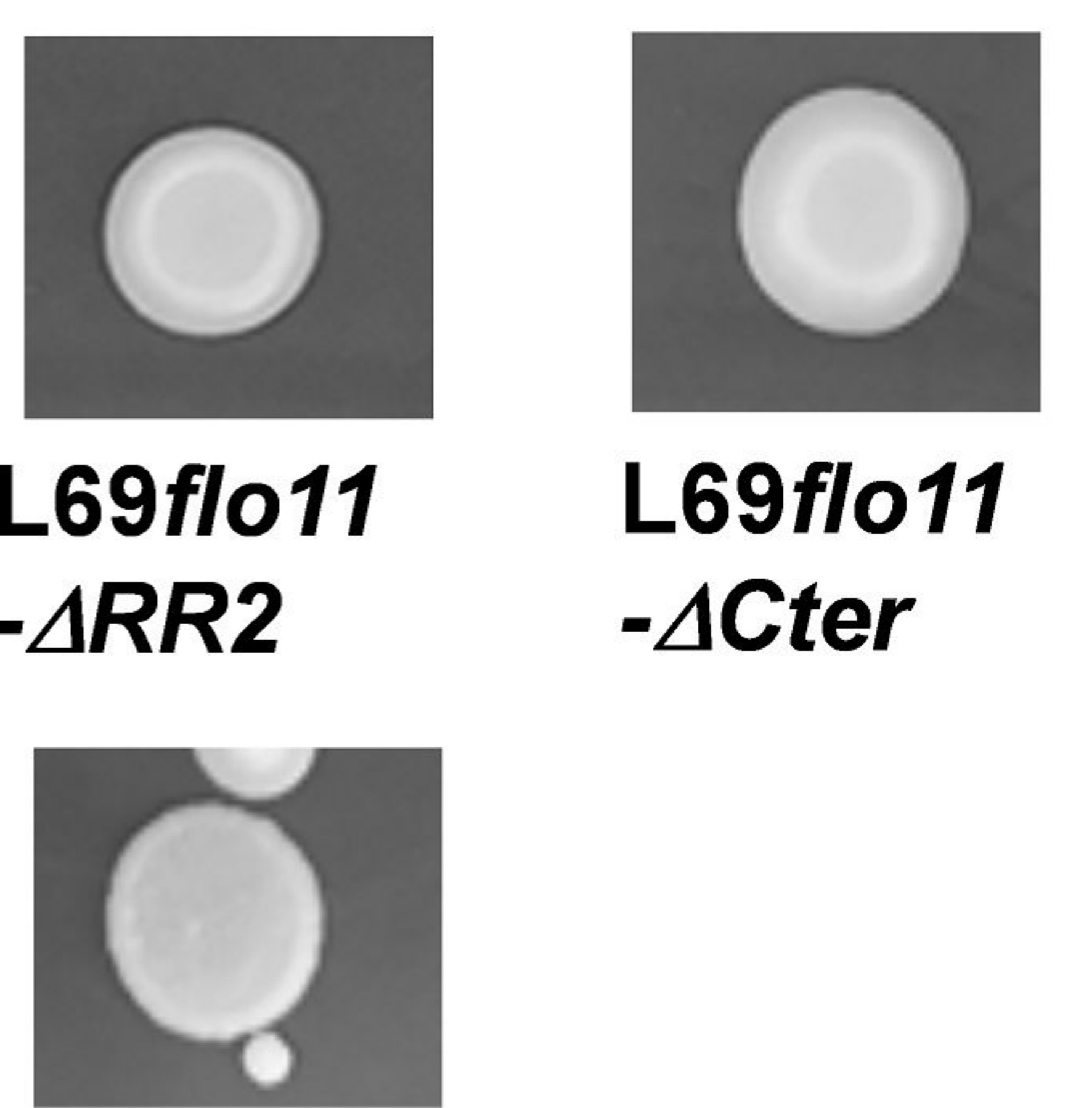

BYflo11A FLO11 ${ }^{B Y}-[R R 2]^{L 69+}$

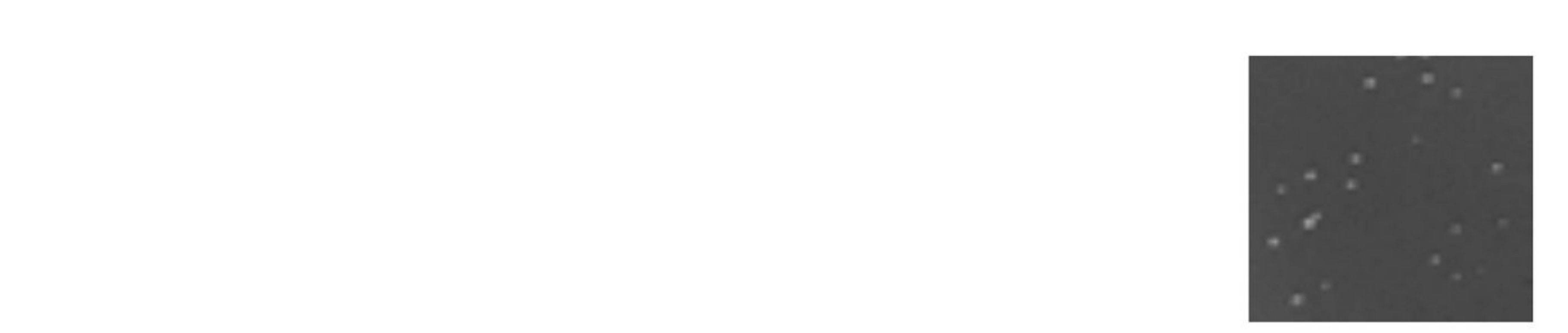

L69flo11 - $\Delta$ Nter

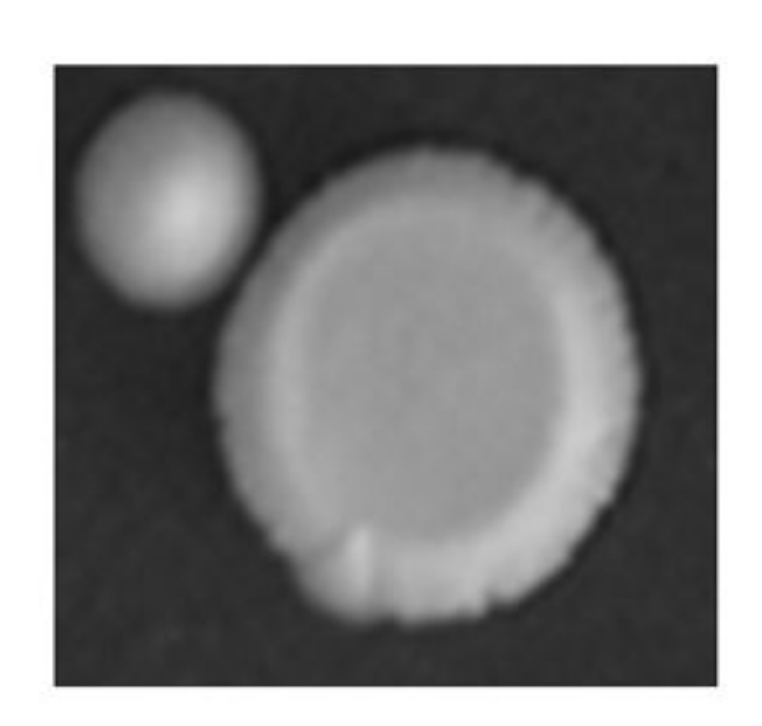

$\Sigma$ flo11

FLO11BY+

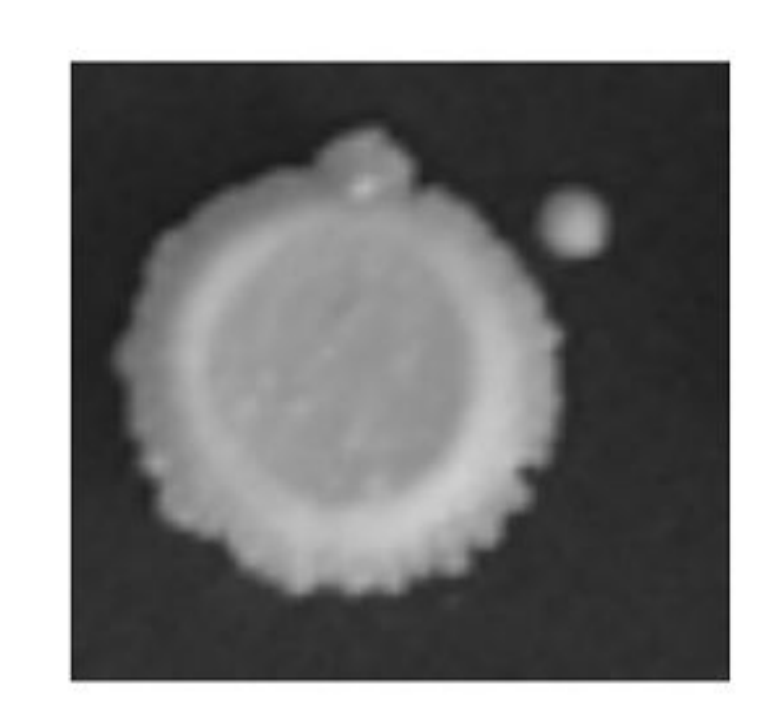

Iflo114

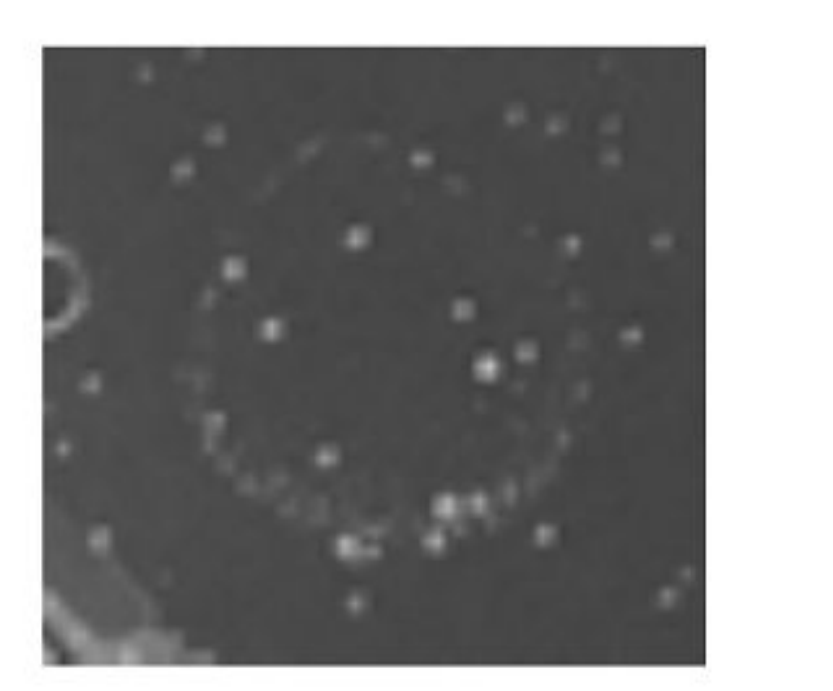

BYflo114

FLO11BY+
FLO11L69+

YPD / After wash

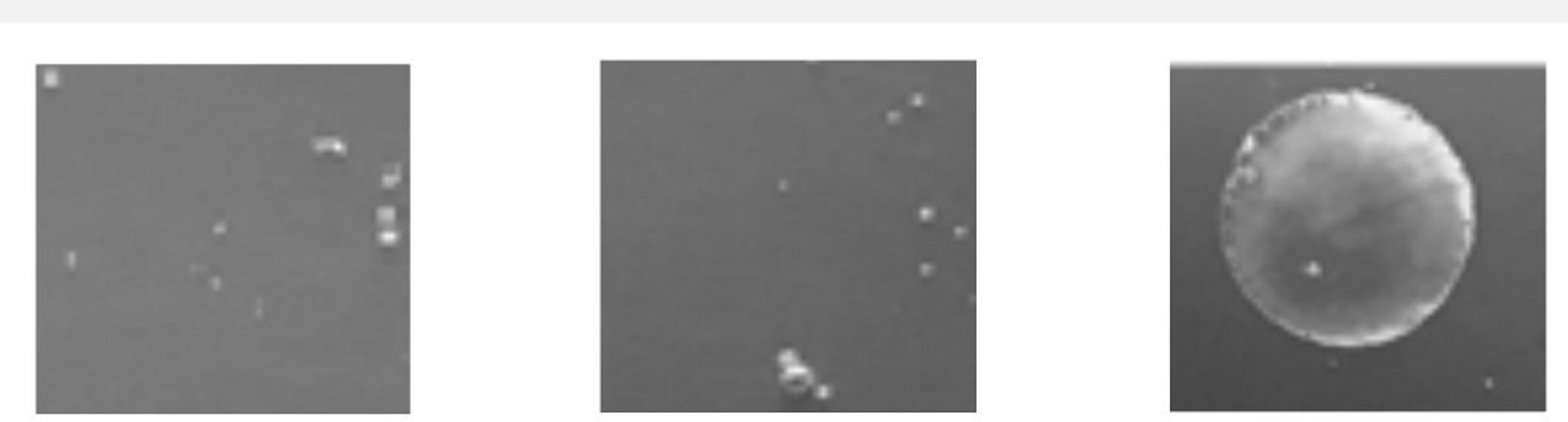

BYflo11A

YSWT3 $\alpha$

¿flo11

L69flo11

BY4741

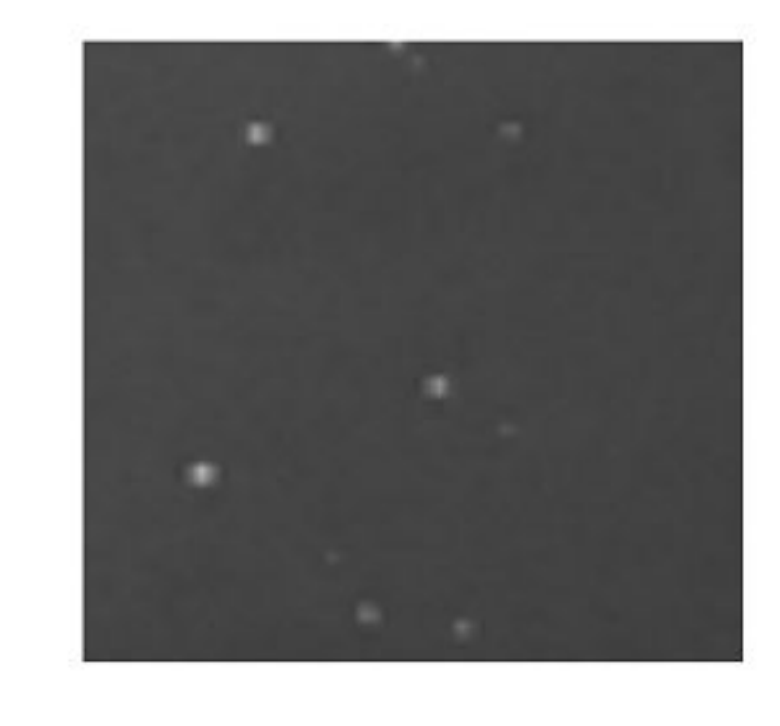

L69flo11

- $\Delta$ Cter

\section{L69flo11}

$-\Delta R R 1$

L69flo11

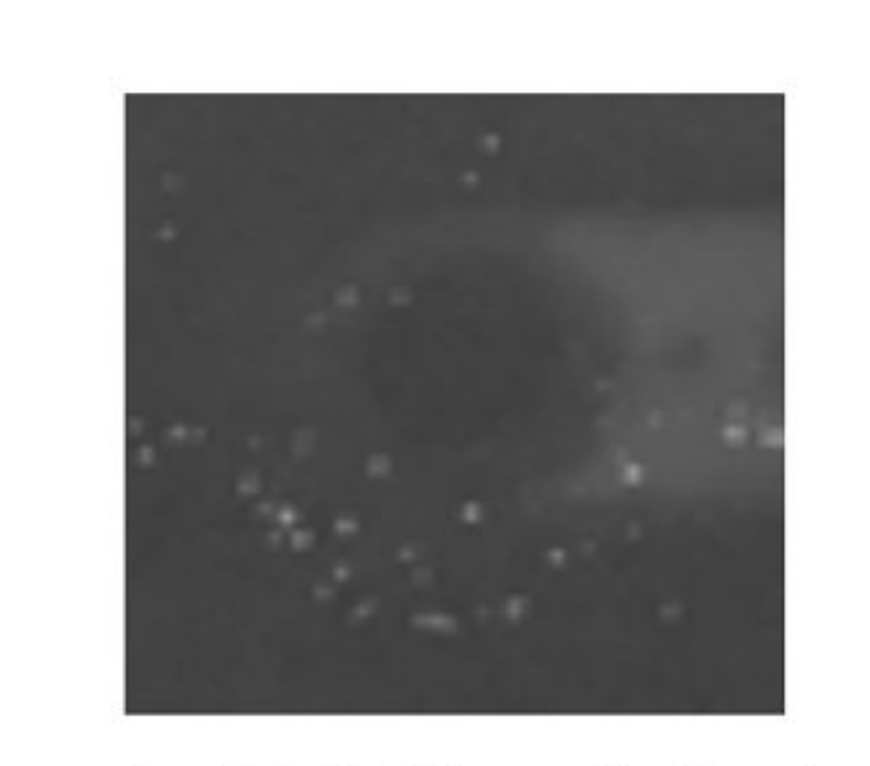

BYflo114

FLO11'69+

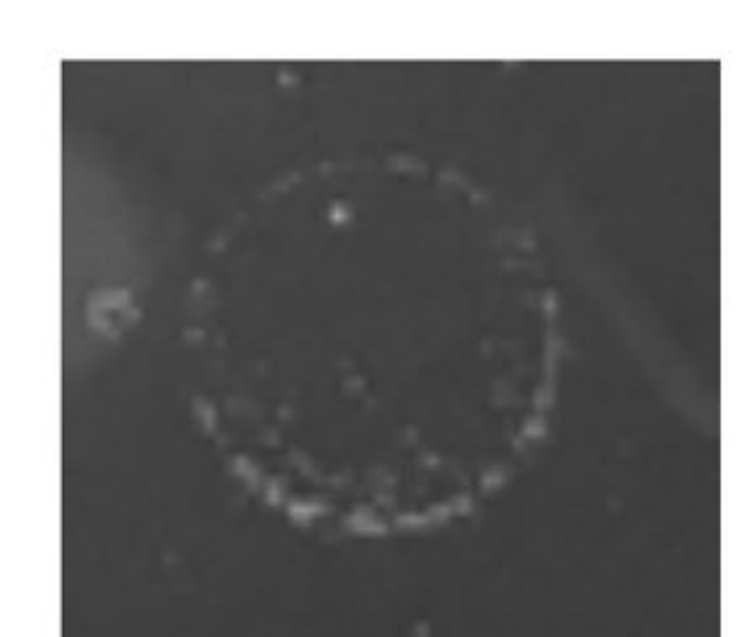

BYflo11A

${ }^{F L O 11^{B Y}}{ }_{-[R R 2]^{L 69+}}$

YNGal / After wash
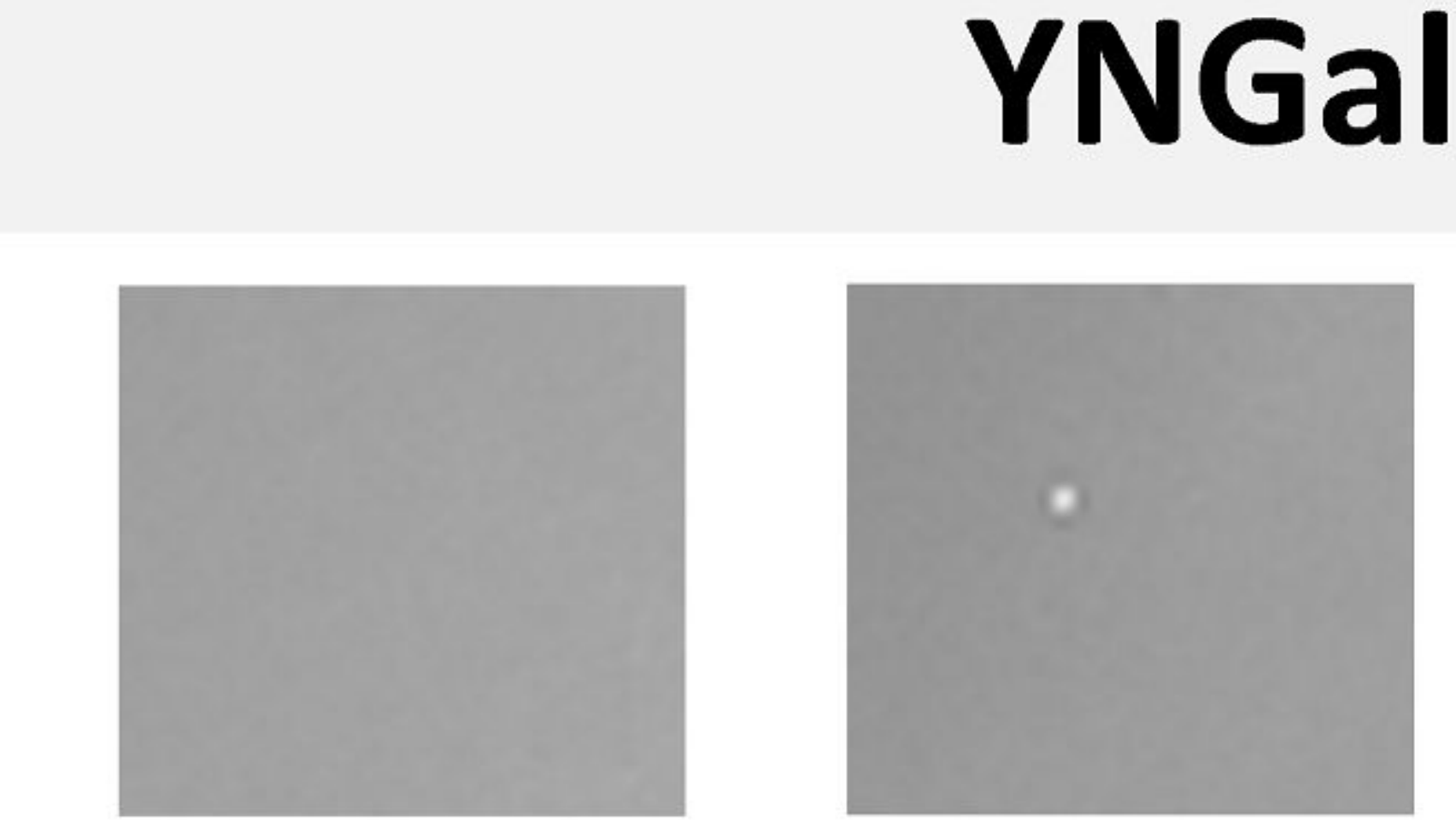

L69flo11A BY4741

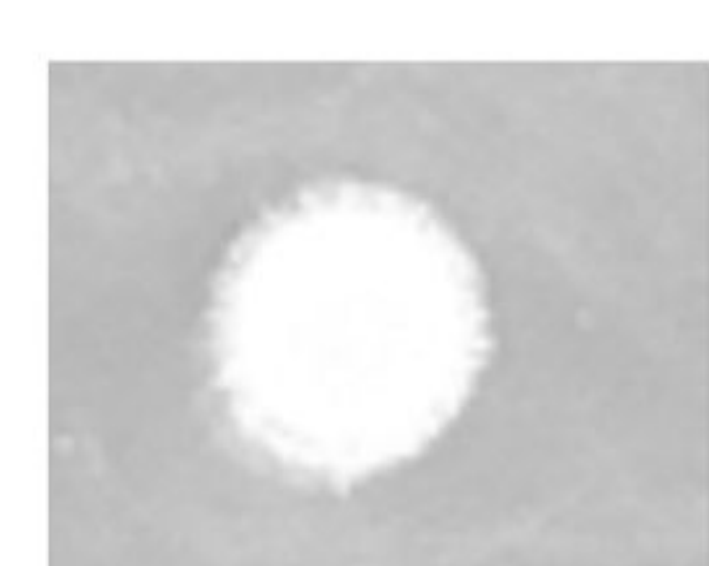

L69flo11 L69flo11 $-\triangle R R 1$
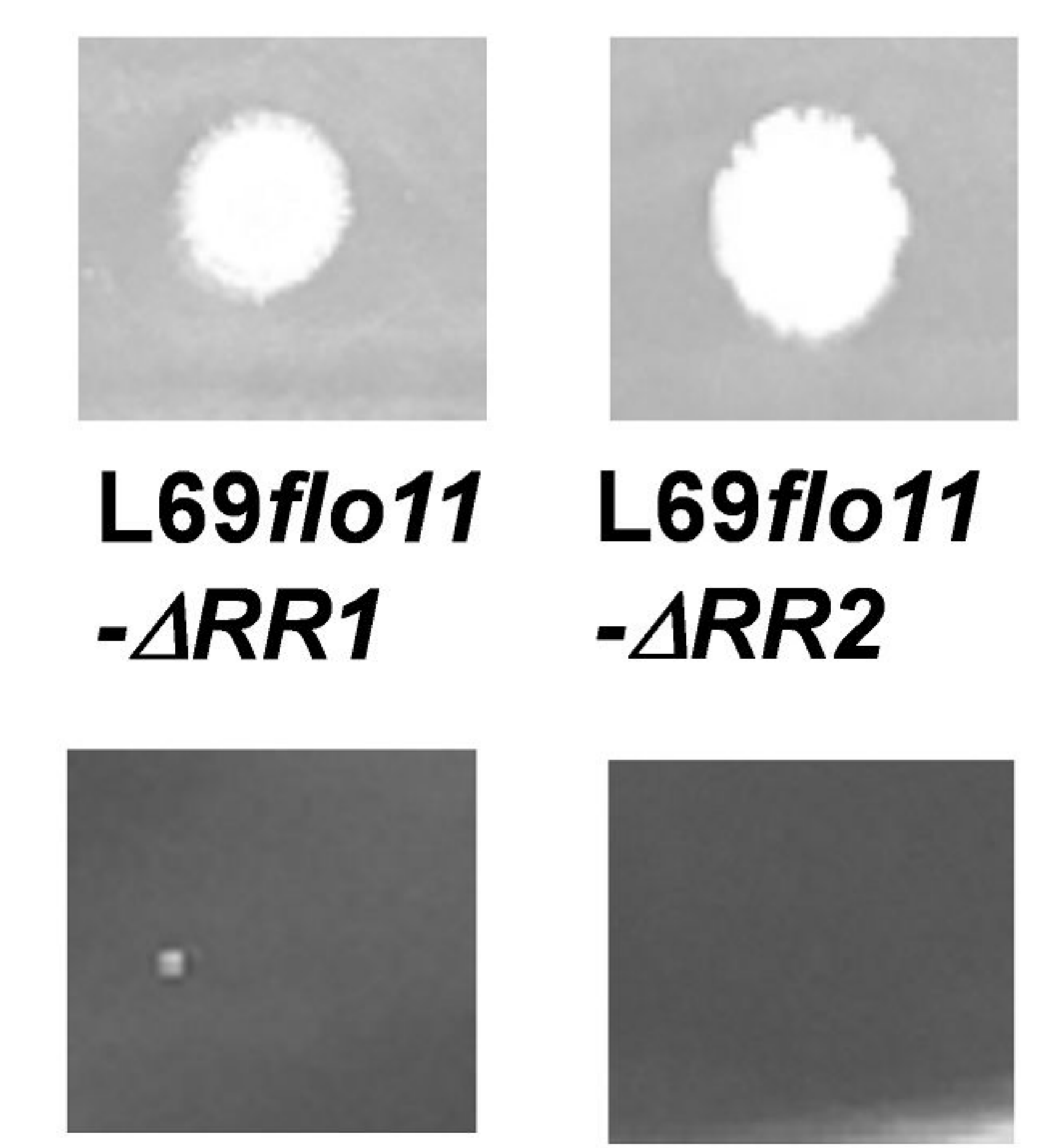

BYflo11ム BYflo11

FLO11 ${ }^{L 69_{+}}+\mathrm{FLO11}^{\mathrm{BY}}{ }_{-[R R 2]^{L 69_{+}}}$

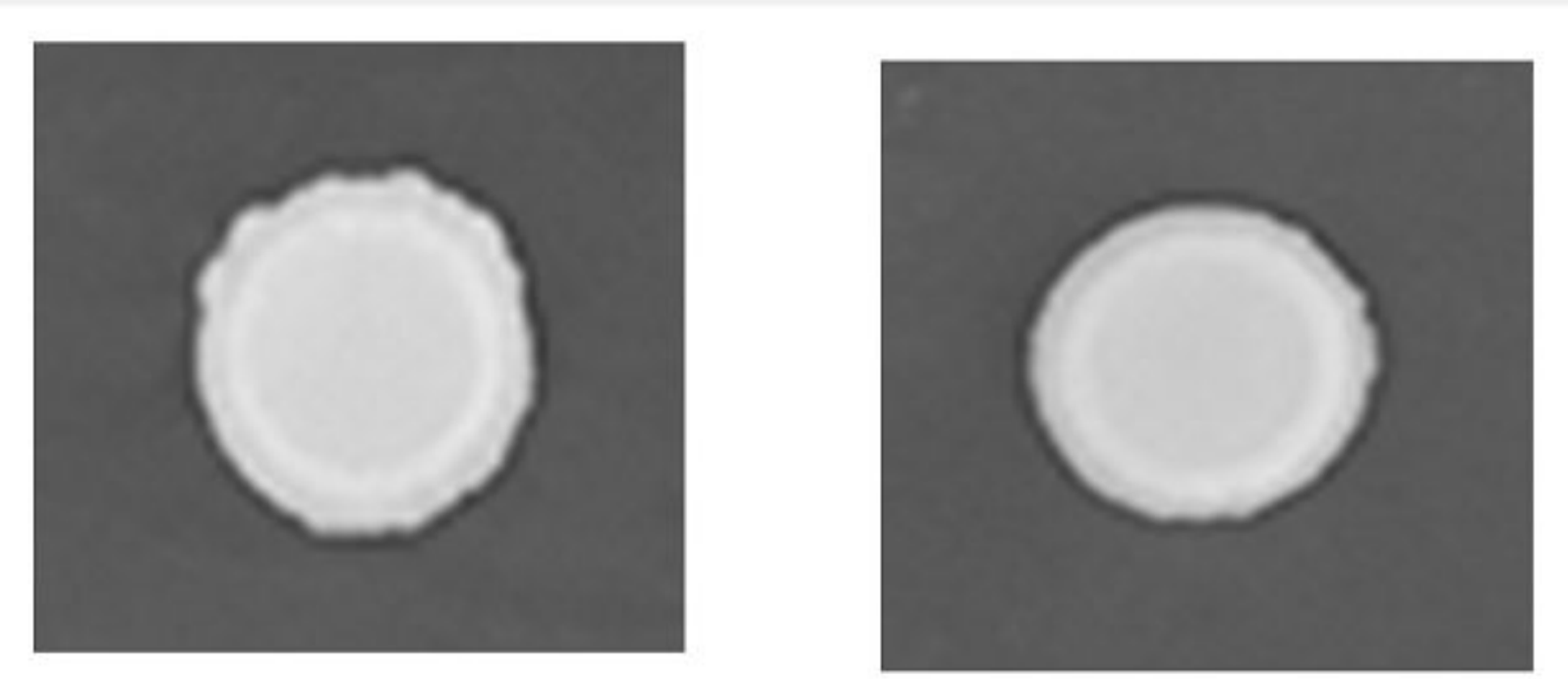

YSWT3 $\alpha \quad \Sigma$ flo11

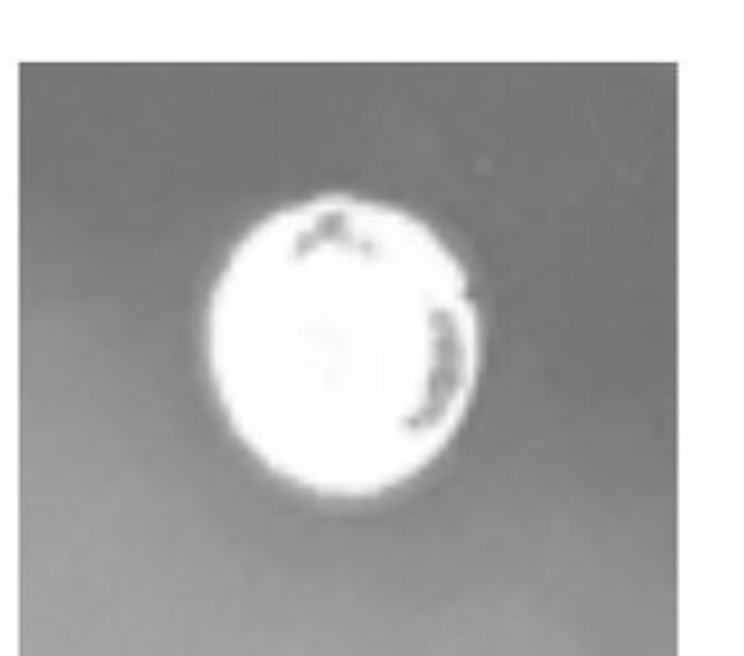

L69

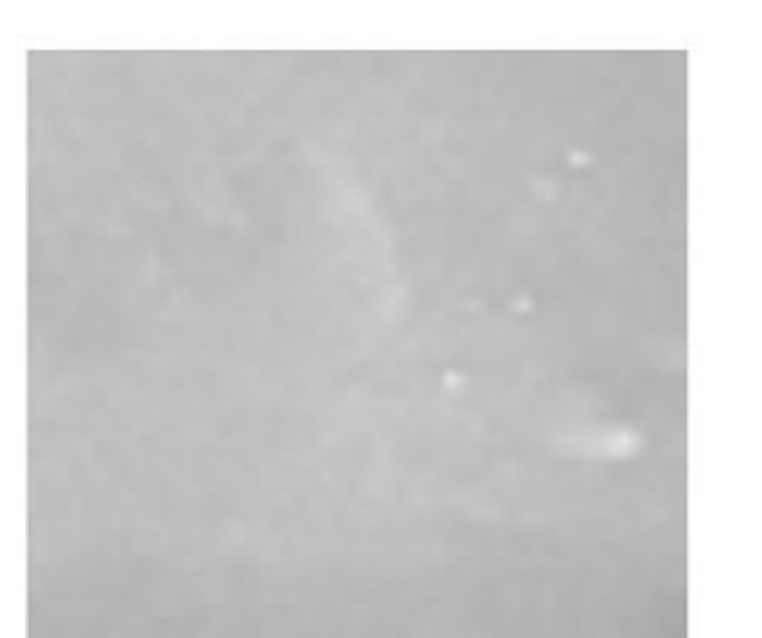

L69flo11 -ANter

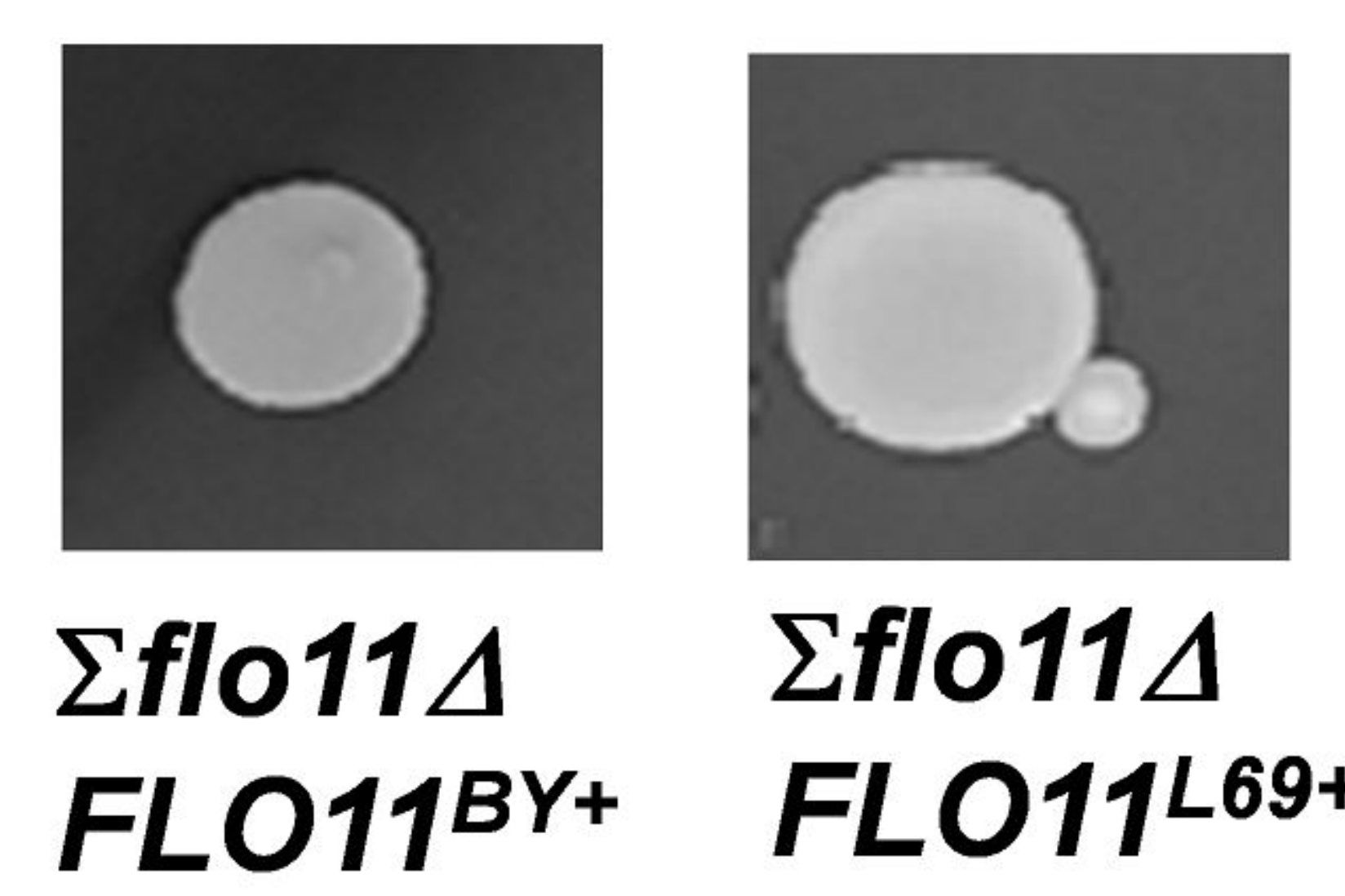

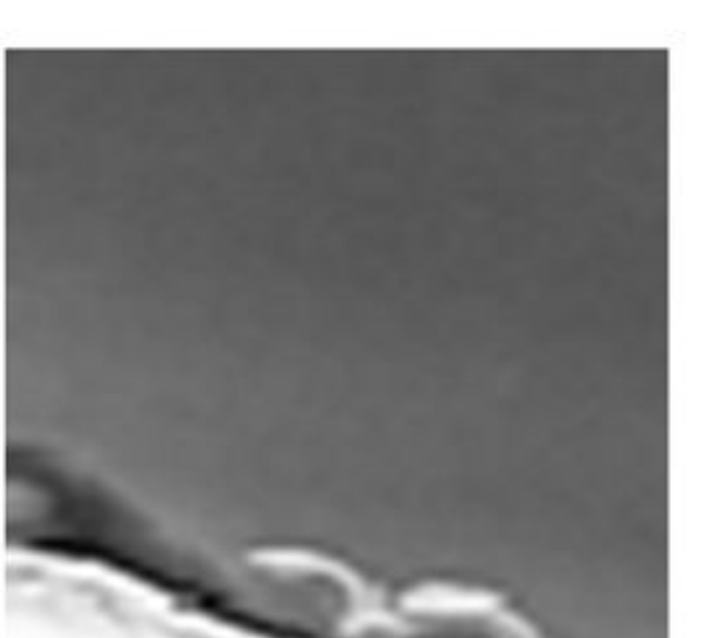

BYflo11A FLO11 ${ }^{B Y}+$

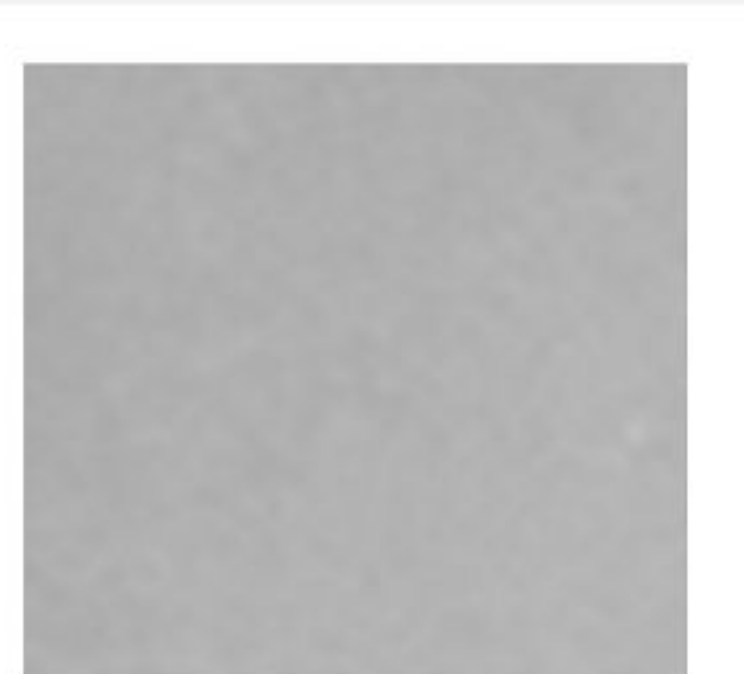

BYflo114

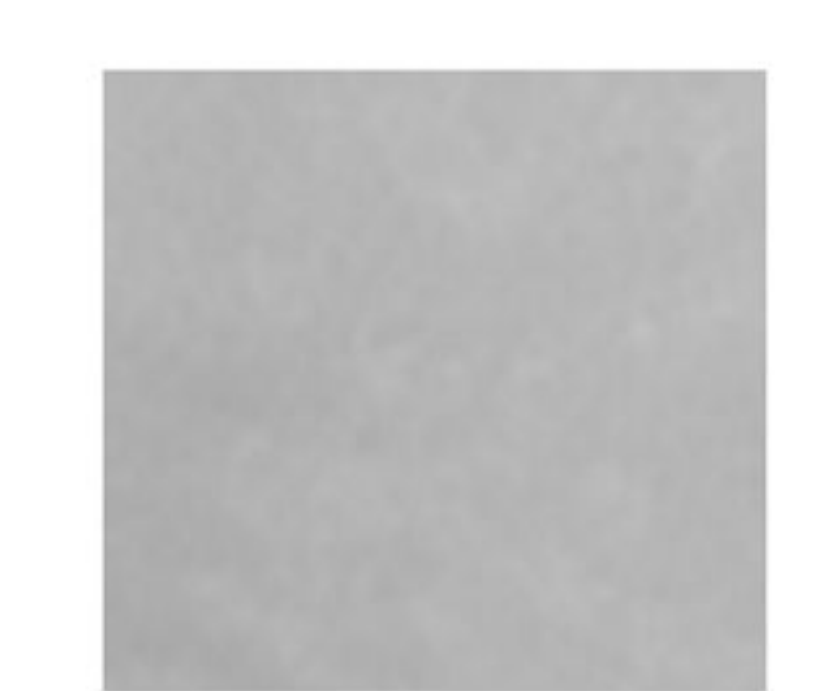

69flo11 - $\Delta$ Cter FLO11BY+ FLO11L69+

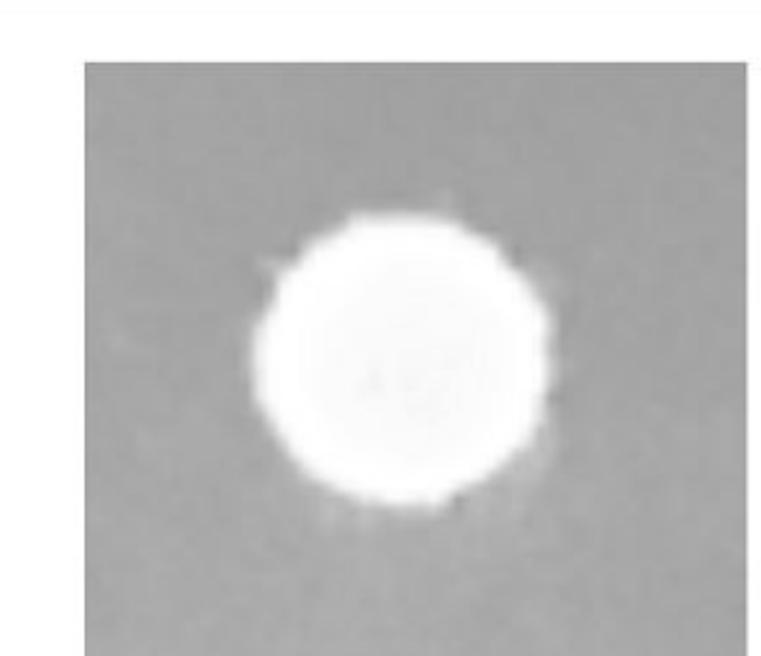

YSWT3 $\alpha$

Iflo11

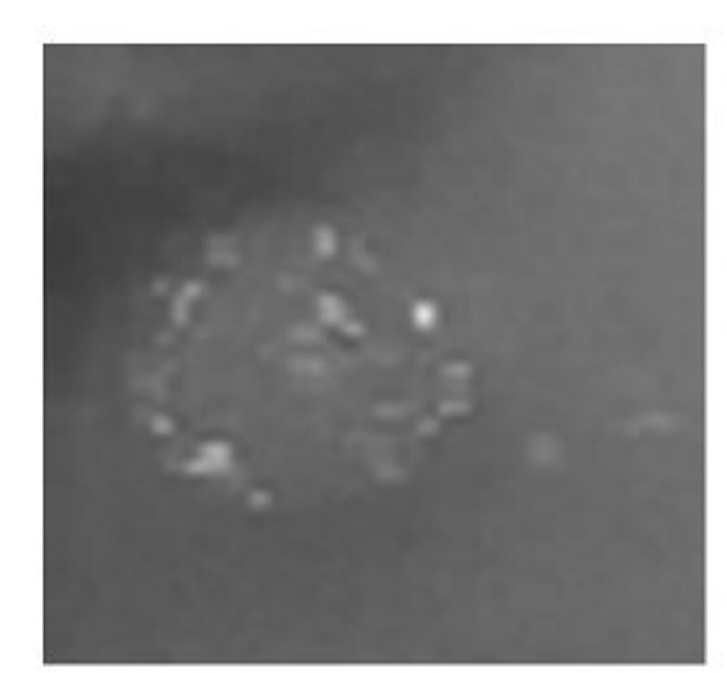

$\Sigma$ flo11 FLO11BY+ FL011L69+ 
The physiological function of Flo11p domains and the particular role of amyloid core sequences of this adhesin in Saccharomyces cerevisiae

\section{Supplementary data}

Clara Bouyx ${ }^{1}$, Marion Schiavone ${ }^{1,2}$, Marie-Ange Teste ${ }^{1}$, Etienne Dague $^{3}$, Nathalie Sieczkowski ${ }^{2}$, Anne Julien ${ }^{2}$ and Jean Marie Francois ${ }^{1^{*}}$

${ }^{1}$ Toulouse Biotechnology Institute (TBI), UMR INSA-CNRS 5504 \& INRA 792; F-31077 Toulouse, France,

${ }^{2}$ Lallemand SAS, 19, rue des briquetiers, 31702 Blagnac, France

${ }^{3}$ CNRS, LAAS F31400 Toulouse, France 


\section{List of Figures and Tables in Supplementary data}

Figure S1: Cell surface analysis of BY4741 and L69 strain using silicon-nitride (Si3N4) AFM cantilevers

Figure S2: Expression levels of FLO11 in L69 and BY4741 strain measured by RT-qPCR.

Figure S3: Sequences alignment of Flo11p from L69, BY4741, $\Sigma 1278 b$ and 133d strains.

Figure S4: Schematic representation of the Flo11 protein sequence from BY4741 strain by Hydrophobic Cluster Analysis (HCA)

Figure S5: Schematic representation of the Flo11 protein sequence from $\Sigma 1278 \mathrm{~b}$ strain by hydrophobic Cluster Analysis (HCA)

Figure S6: Schematic representation of the Flo11 protein sequence from L69 strain by Hydrophobic Cluster Analysis (HCA)

Figure S7: Schematic representation of the Flo11 protein sequence from flor yeast strain 133d by Hydrophobic Cluster Analysis (HCA)

Figure S8: Cell surface analysis of the L69flo11- $\Delta$ Nter strain using silicon-nitride (Si3N4) AFM cantilevers.

Figure S9: Cell surface analysis of the L69flo11- $\Delta$ Cter strain using silicon-nitride (Si3N4) AFM cantilevers.

Figure S10: Cell surface analysis of L69flo11- $\triangle R R 1$ using silicon-nitride (Si3N4) AFM cantilevers.

Figure S11: Effect of Thioflavine S on the Flo11p-dependent cell-cell aggregation

Figure S12: Assay of velum formation

Figure S13: Invasive growth in agar by different yeast strains, and impact of the culture medium and of the domains of FLO11p on this phenotype

Figure S14: Sensitivity of L69 and L69 mutant strains to Caffeine and Calcofluor White (CFW) drugs.

Table S1. TANGO software analysis of $\beta$-aggregation motifs in Flo1, Flo5, Flo9, Flo10 and Flo11 protein from Saccharomyces cerevisiae S288c strain

Table S2. Search for intragenic repeats using EMBOSS ETANDEM software

Table S3. Search for $\beta$-aggregation prone sequence in the different Flo11 proteins using

TANGO software (http://tango.crg.es/)

Table S4. Yeast strains used or constructed in this study.

Table S5: Plasmids constructed in this work

Table S6. Oligonucleotides used in this study 
A

B

Height image

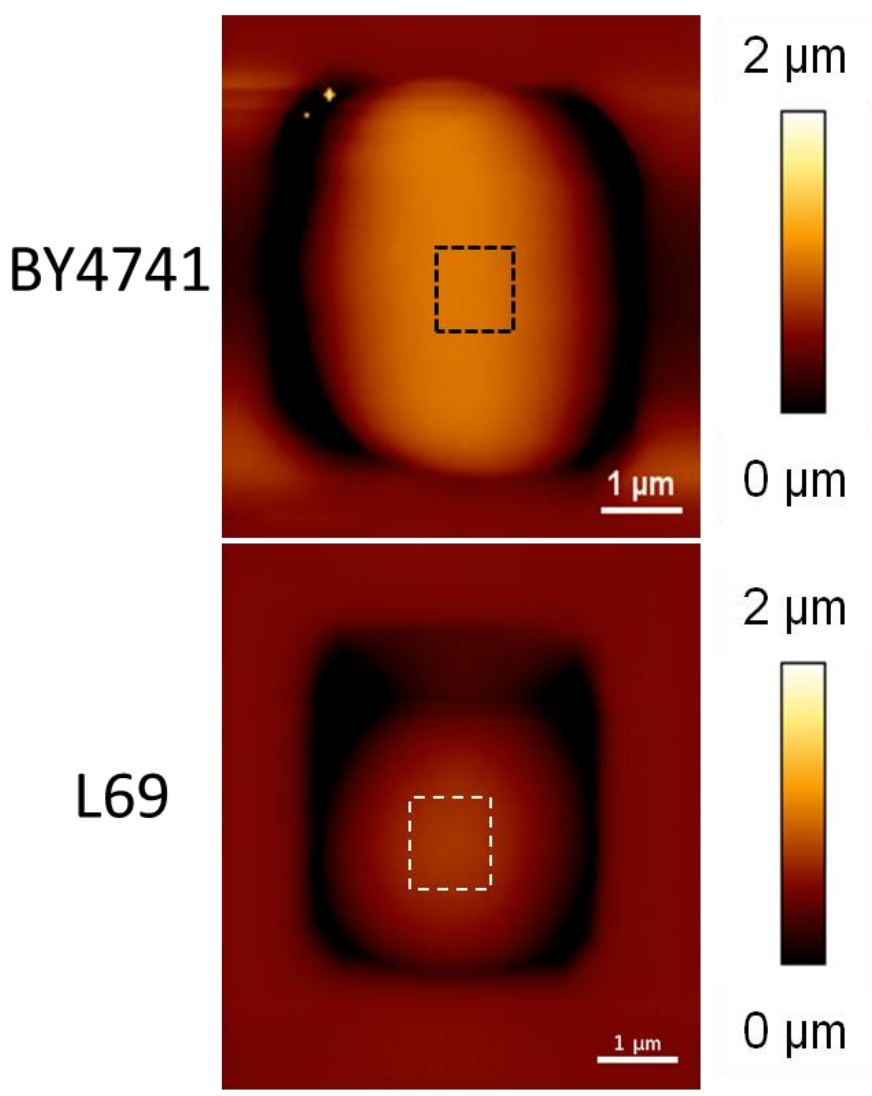

Adhesion image
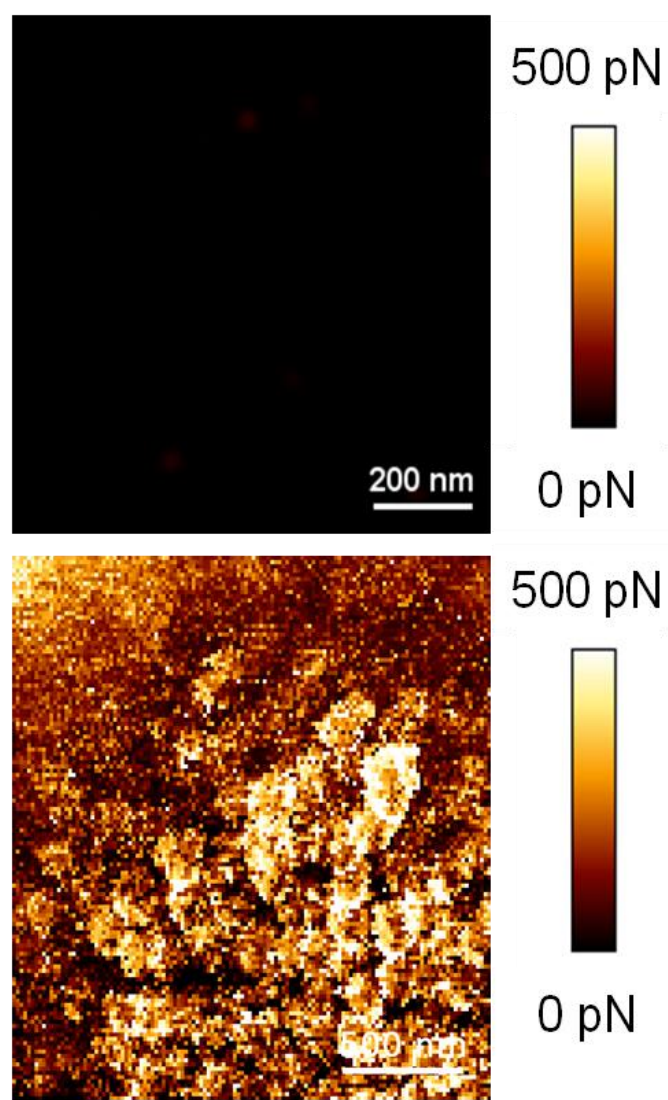

Figure S1: Cell surface analysis of BY4741 and L69 strain using silicon-nitride (Si3N4) AFM cantilevers. A single cell from strain BY4741 and L69 was trapped in the PDMS chamber as described in Material and Methods. AFM height image (z-scale: $2 \mu \mathrm{m}$ ) (A) and adhesion image (z-scale: $500 \mathrm{pN}$ ) analyzed on the hatched square of the height image $(B)$ were recorded in $Q$ I mode. 
A

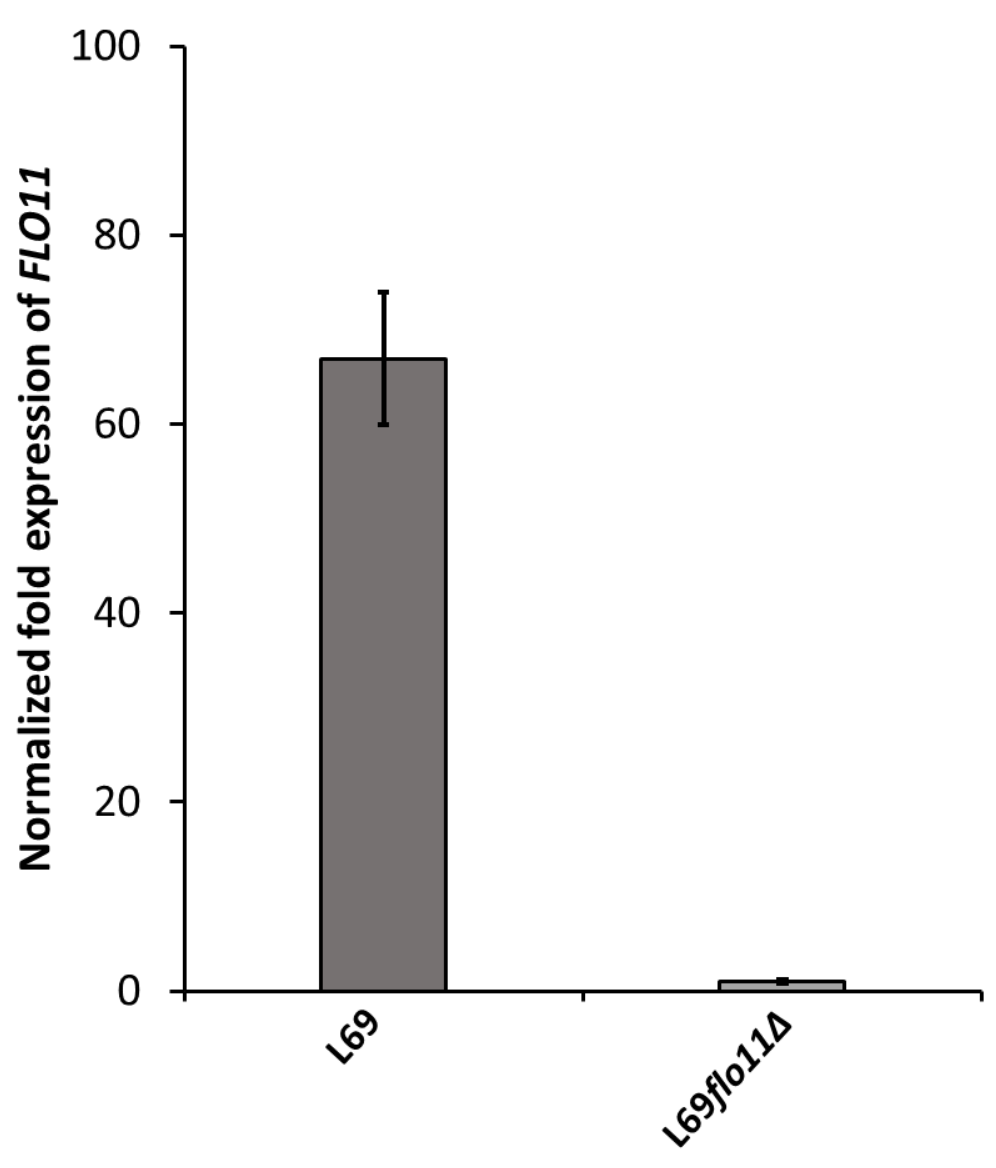

B

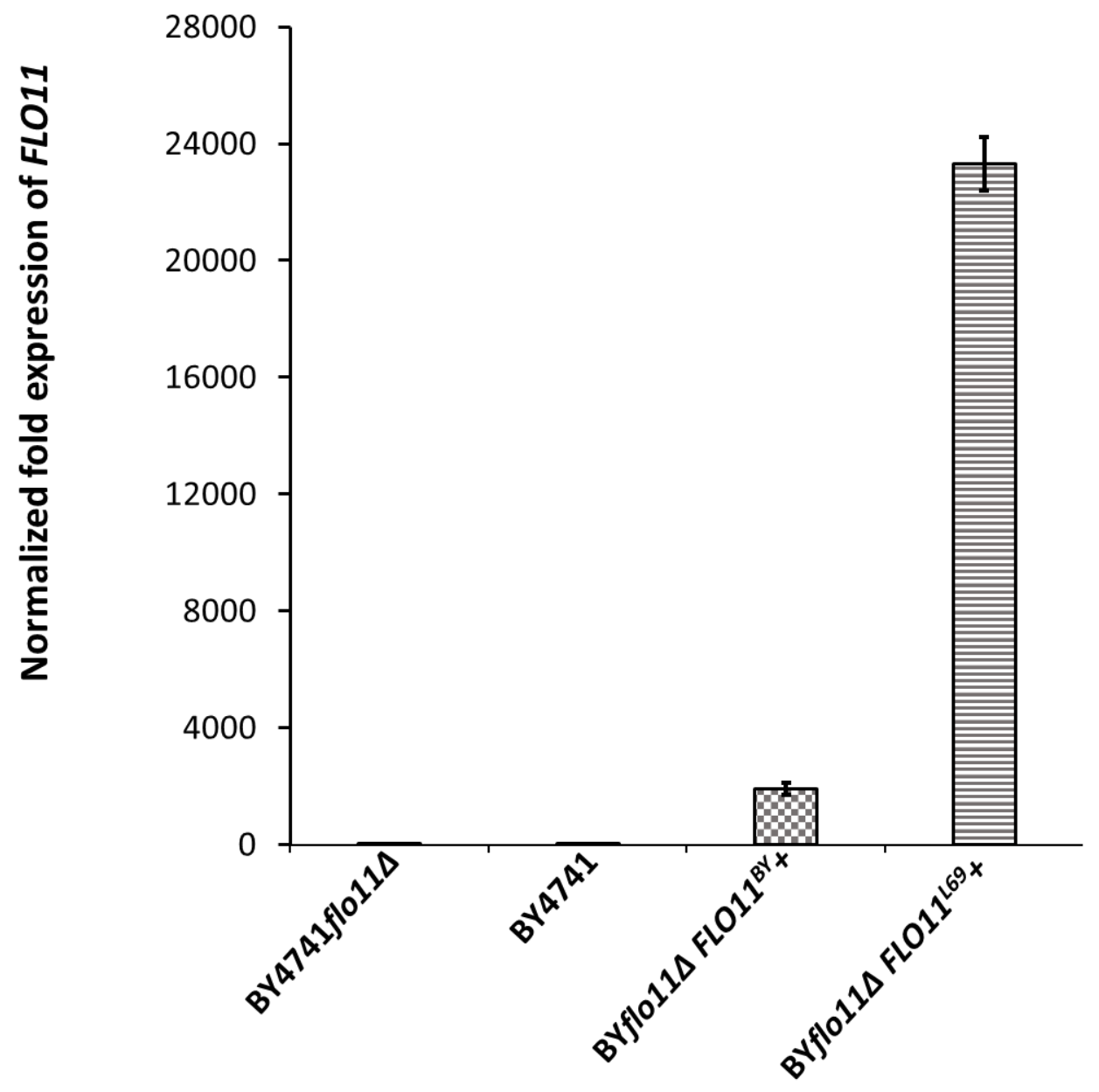

Figure S2: Expression levels of FLO11 in L69 and BY4741 strain measured by RT-qPCR. Transcript levels of FLO11 were determined in exponential cultures of L69 and BY4741 strains on YPD (A) or on YNGal supplemented with required amino acids, uracil and adenine at $0.1 \%$ (w/v), except for BY4741 transformed with $F L O 11^{B Y}$ or FLO11 ${ }^{L 69}$ for which uracil was omitted. The transcripts was normalized to internal reference genes TAF10 and UBC6. 


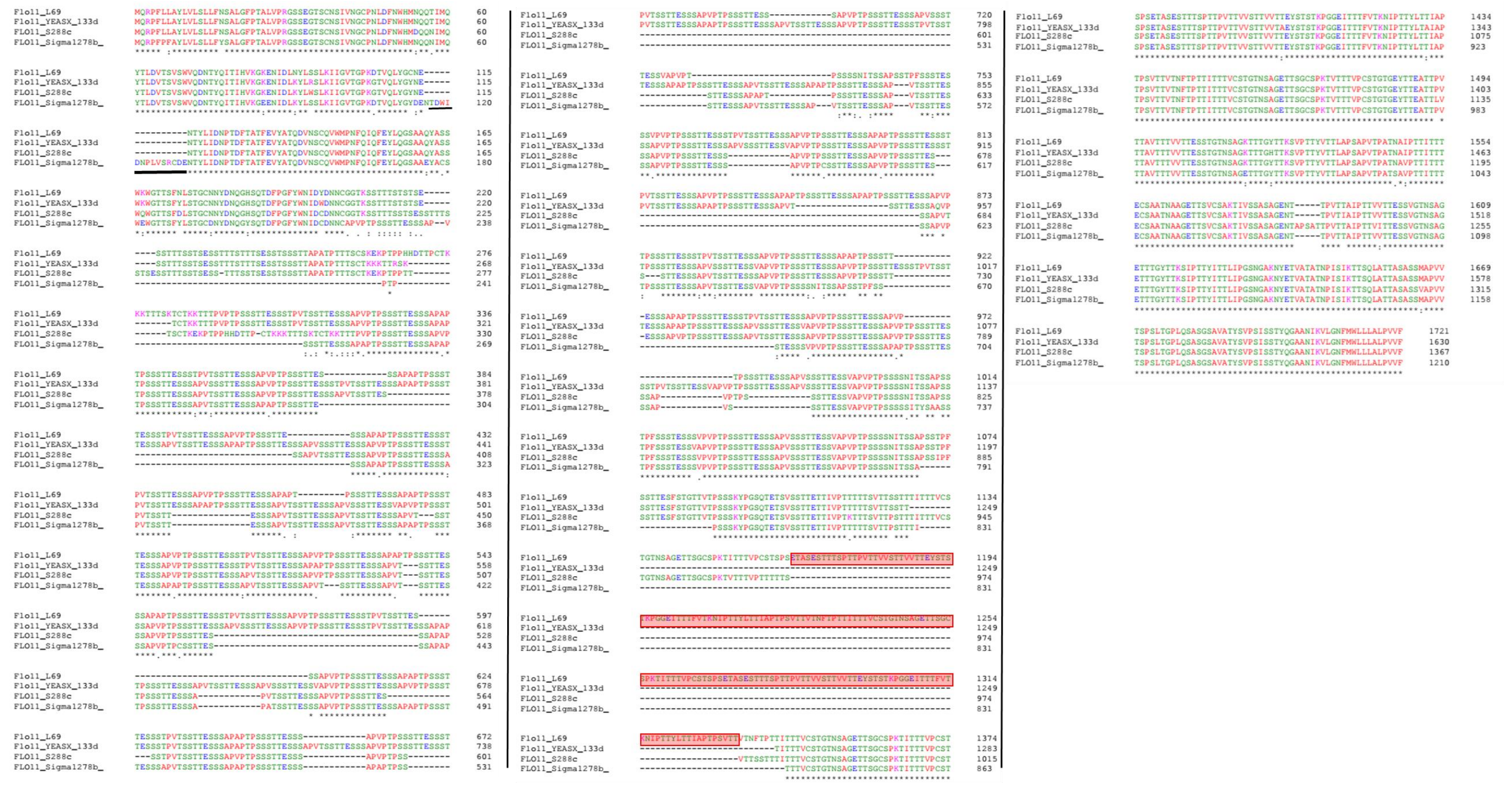

Figure S3: Sequences alignment of Flo11p from L69, BY4741, $\Sigma 1278 \mathrm{~b}$ and 133d strains. The Flo11p sequences were aligned with Clustal Omega. The sequence of Flo11p from L69 strain was retrieved from the genome sequence of this strain (Lallemand Inc, unpublished data), whereas Flo11p from BY4741, $\Sigma 1278 \mathrm{~B}$ and 133d were retrieved from public repository data at NCBI. Boxed in red indicate amino acid sequence (RR2) present in Flo11p of L69 strain and absent in Flo11p of the other strains. Asterisks indicate positions with identical amino acids, dots indicate single amino acid difference between amino acid sequences. Black bar highlights the unique 15 amino acid sequence in the Flo11 of $\Sigma 1278 b$ strain 
Flo11p $5288 c(1367$ aa)
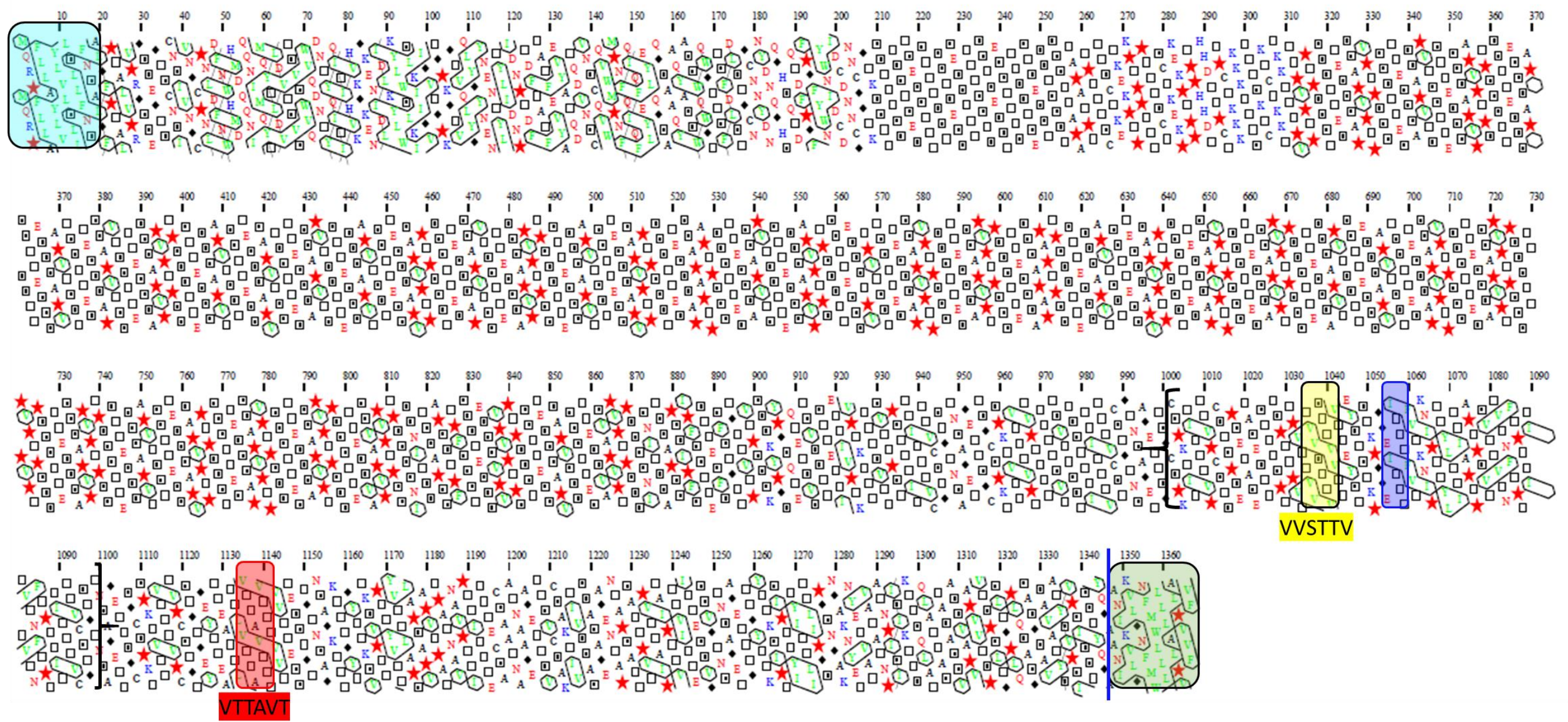

Figure S4: Schematic representation of the Flo11 protein sequence from BY4741 strain by Hydrophobic Cluster Analysis. The Flo11p from BY4741 strain is shown with the Nterminal secretion signal sequences boxed in blue determined with SignalP-4.1 server and the C-terminal GPI addition signals using PredGPI tool, boxed in green with a blue line indicating the omega-site position (GPI signal anchorage to cell wall $\beta$-glucan) which includes a $\beta$-aggregation motif FMWLLA. Amyloid core sequences 'VVSTTV' or 'VTTAVT' are highlighted in yellow and red boxes. The ITTTFV $\beta$-aggregation motif that specifically follows the first amyloid forming sequence is shown as violet box. Between bracket is the amino acids sequence that is found times repeated in Flo11p of L69 strain. 


\section{Flo11p $p^{\Sigma 1278 b}(1210$ aa)}
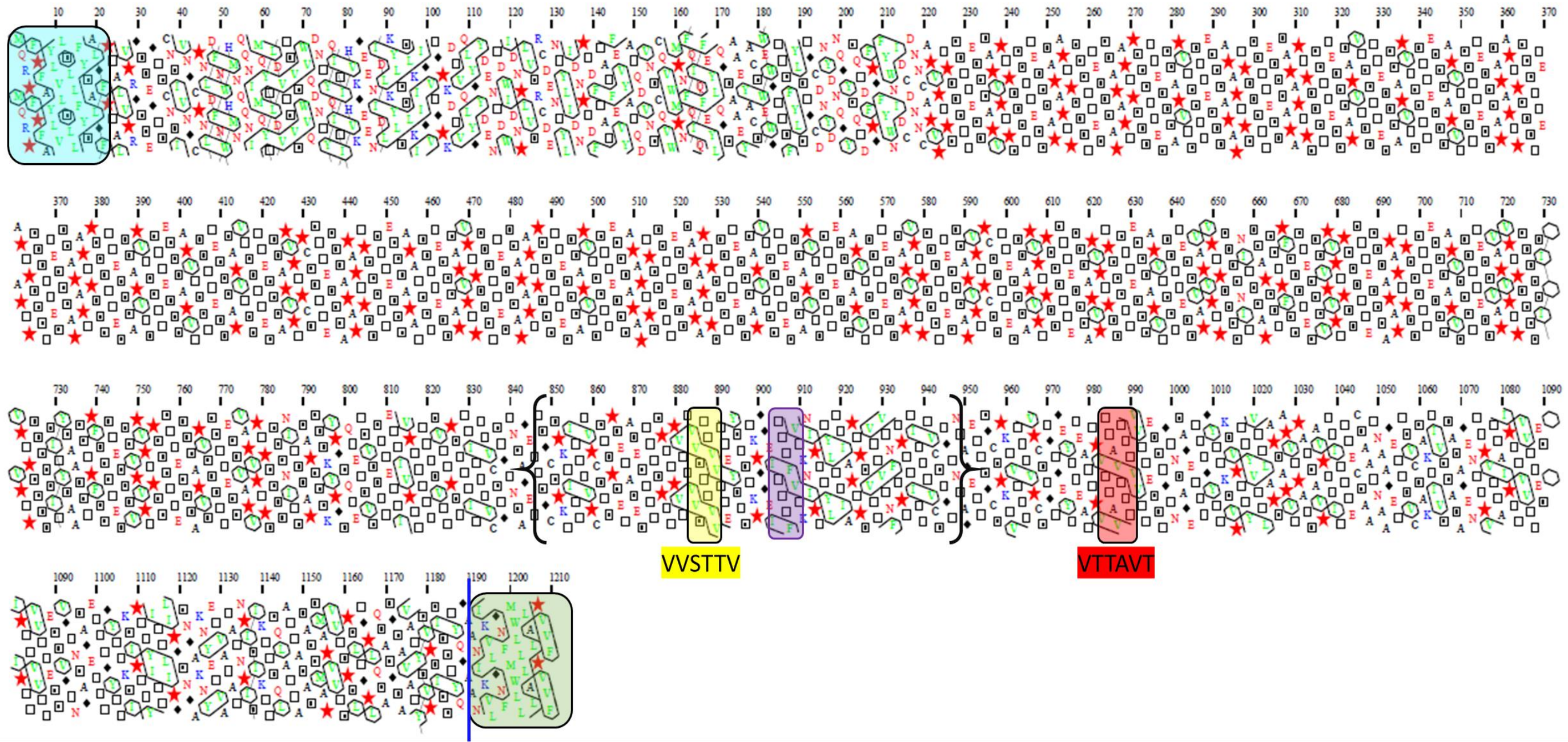

Figure S5: Schematic representation of the Flo11 protein sequence from $\Sigma 1278 b$ strain by hydrophobic Cluster Analysis. Same description as in Fig.S4 
Flo11p $^{\text {L69 }}(1722$ aa)

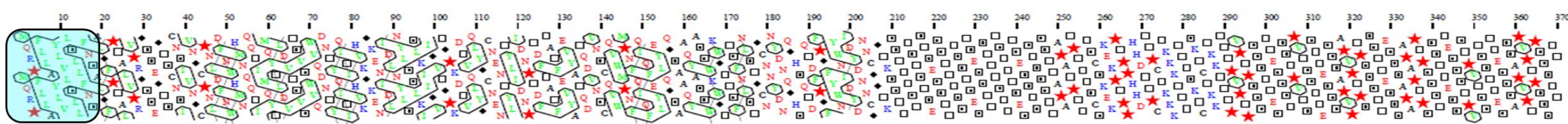

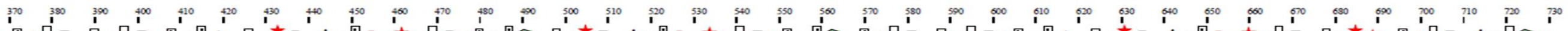

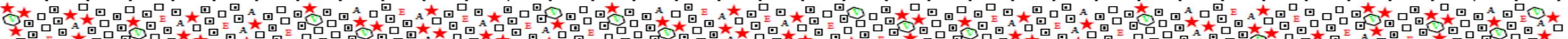

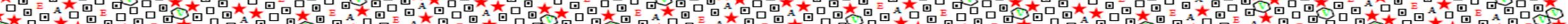

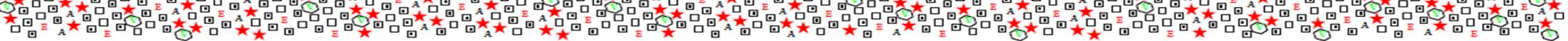

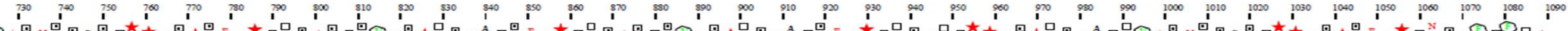

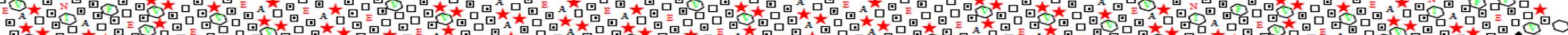

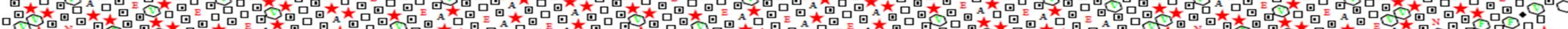

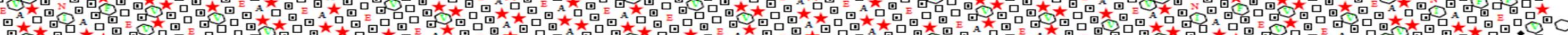
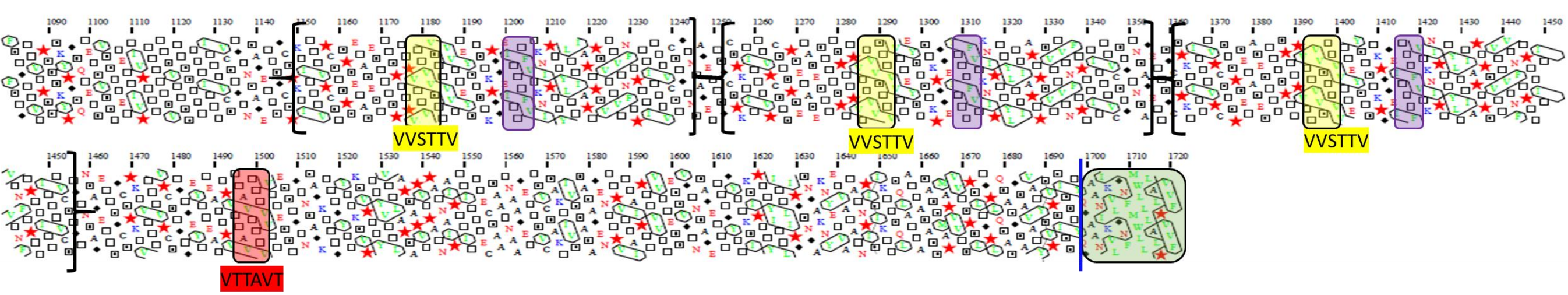

Figure S6: Schematic representation of the Flo11 protein sequence from L69 strain by Hydrophobic Cluster Analysis ( HCA). Same as in Figure S4. Notice the presence of two additional amyloid-forming sequences, together with two additional $\beta$-aggregation motifs ITTTFV in this protein that are not present in Flo11p of all the other strains. In brackets is indicated the region that has been duplicated in the Flo11p to yield these two additional amyloid $\beta$-aggregation prone sequences in the Flo11p of L69 strain. 


\section{Flo11p $p^{133 d}$ (1630 aa)}

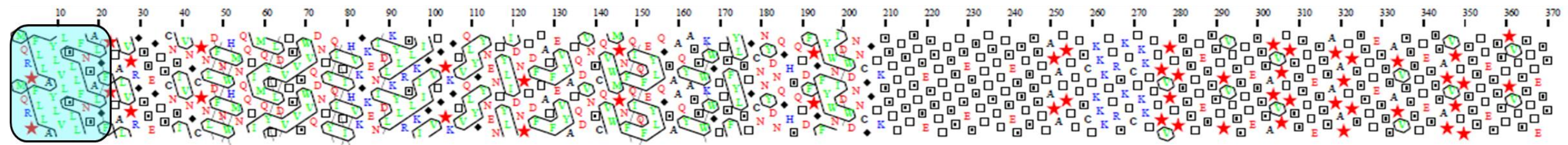

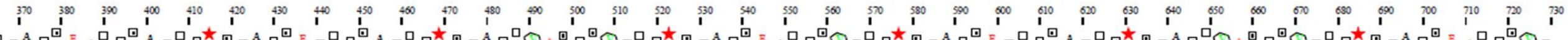

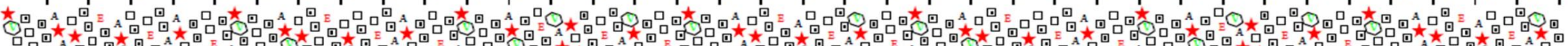

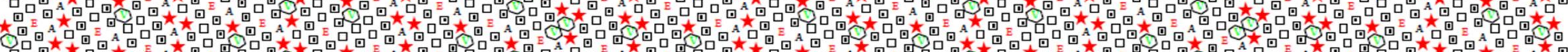

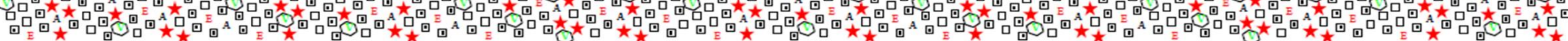

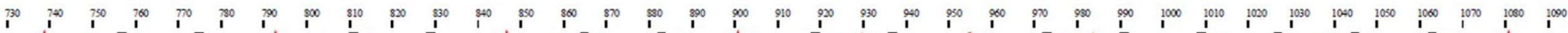

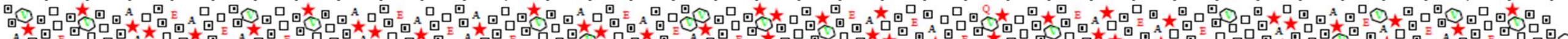

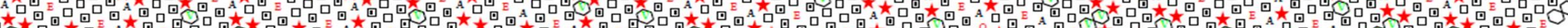

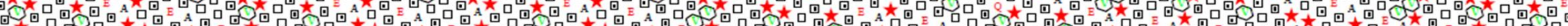

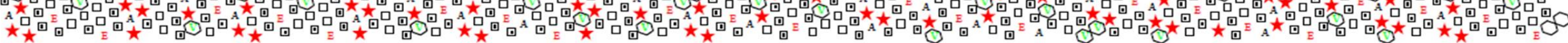

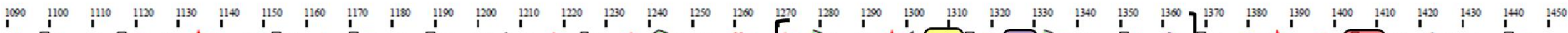

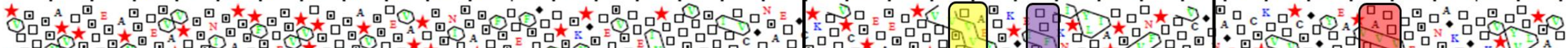

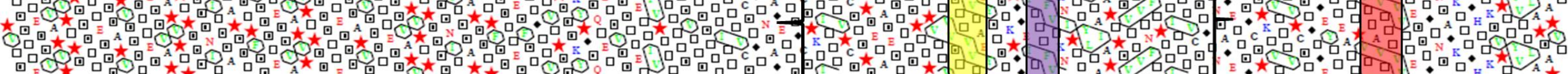

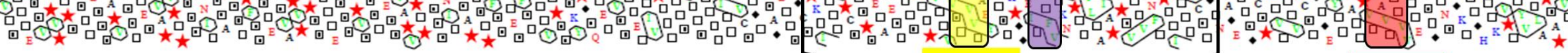

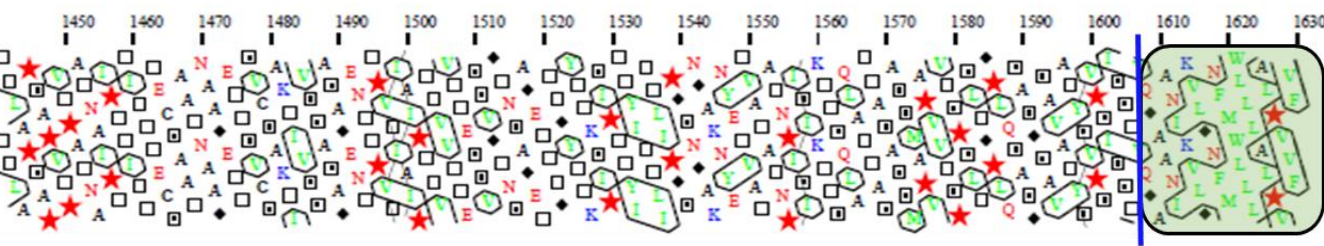
VVSTTV

Figure S7: Schematic representation of the Flo11 protein sequence from flor yeast strain 133d by Hydrophobic Cluster Analysis. Same as in Fig. 4 


\section{L69flo11- $\Delta$ Nter}
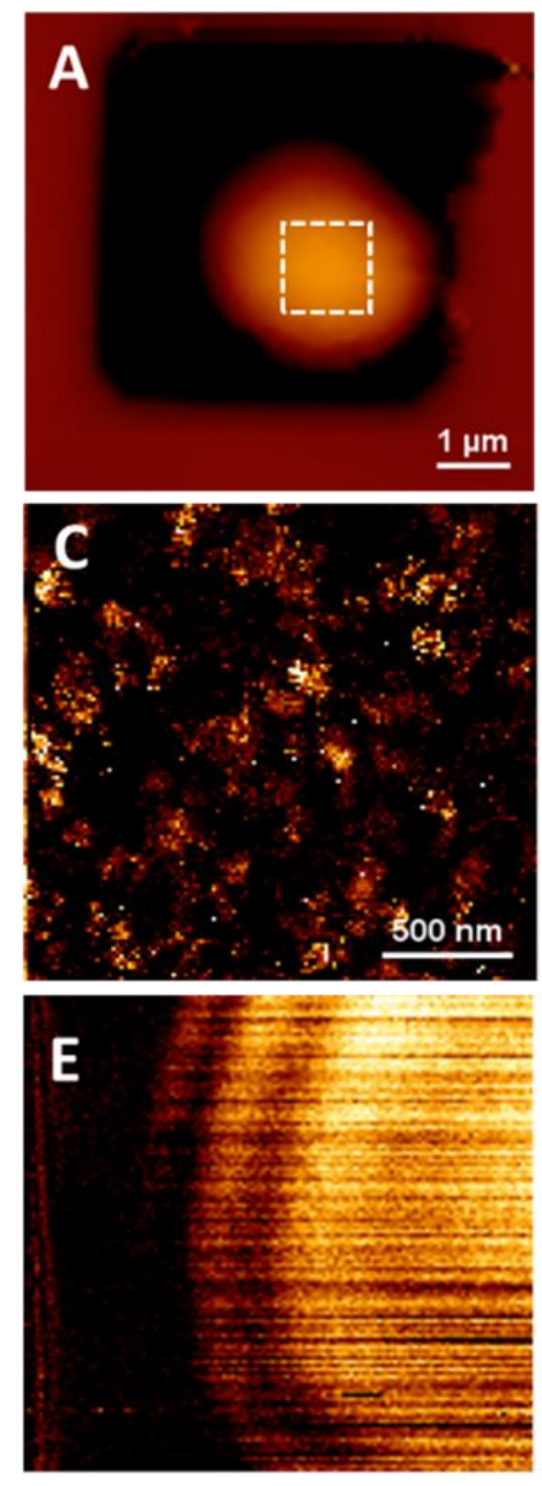
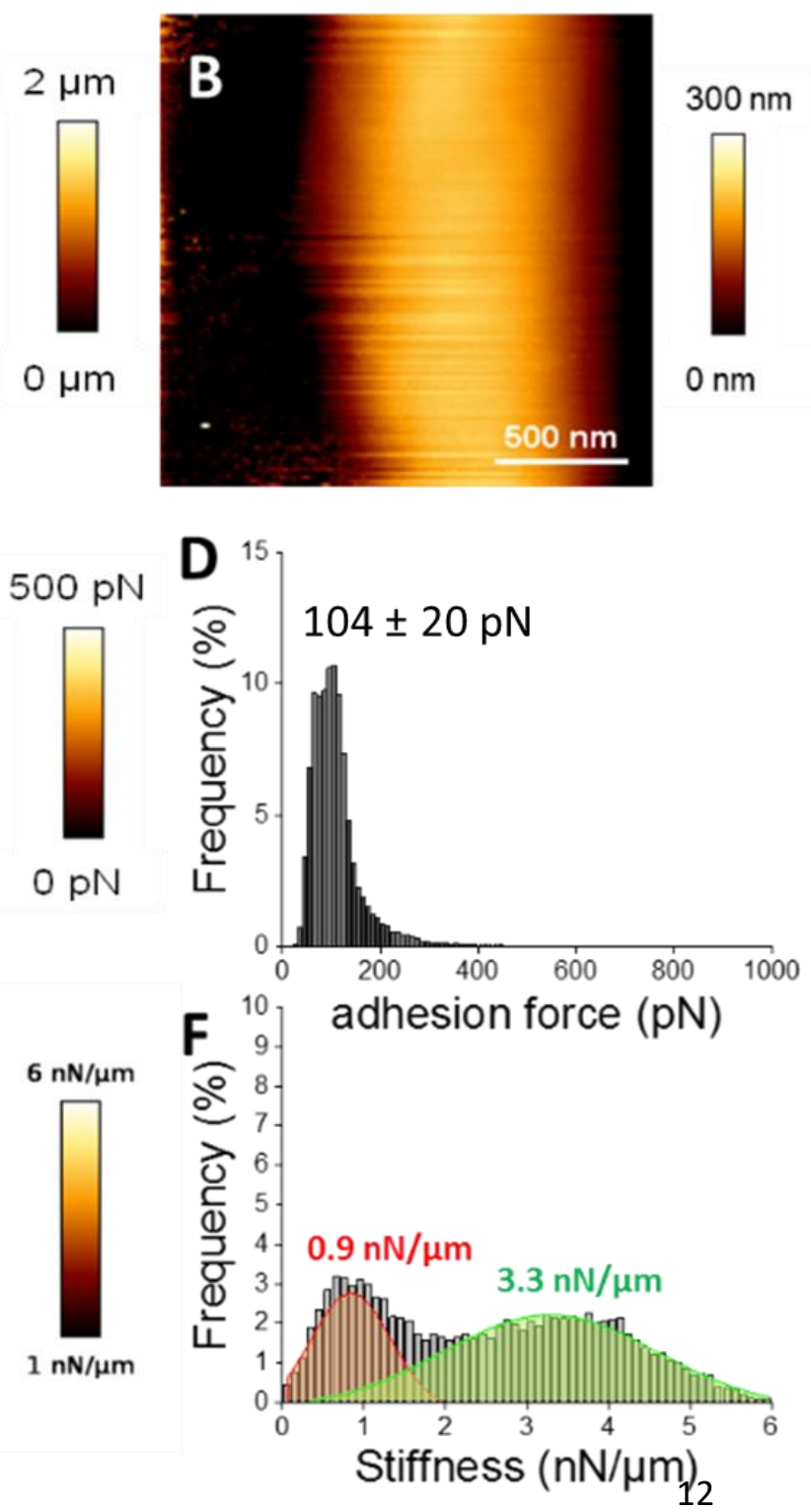

Figure S8: Cell surface analysis of the L69flo11$\Delta N$ ter strain using siliconnitride (Si3N4) AFM cantilevers. AFM height image $(A, B)$, adhesion image from the hatched square in $A$ (C) and stiffness image (E) of a single yeast cell from L69 flo11- $\Delta$ Nter trapped in a PDMS chamber is shown. In (D) is reported the adhesion force histogram obtained from 1024 force-distance curve recorded in QI ${ }^{\mathrm{TM}}$ mode on the area of cell surface illustrated in (C). In (F) is reported the stiffness histogram from 1024 forcedistance curve recorded in $\mathrm{Q}^{\mathrm{TM}}$ mode. See Fig 2 and Material \& Method for the visualization and quantitative determination of adhesion force and stiffness from the force-distance curves. 


\section{L69flo11- $\Delta$ Cter}
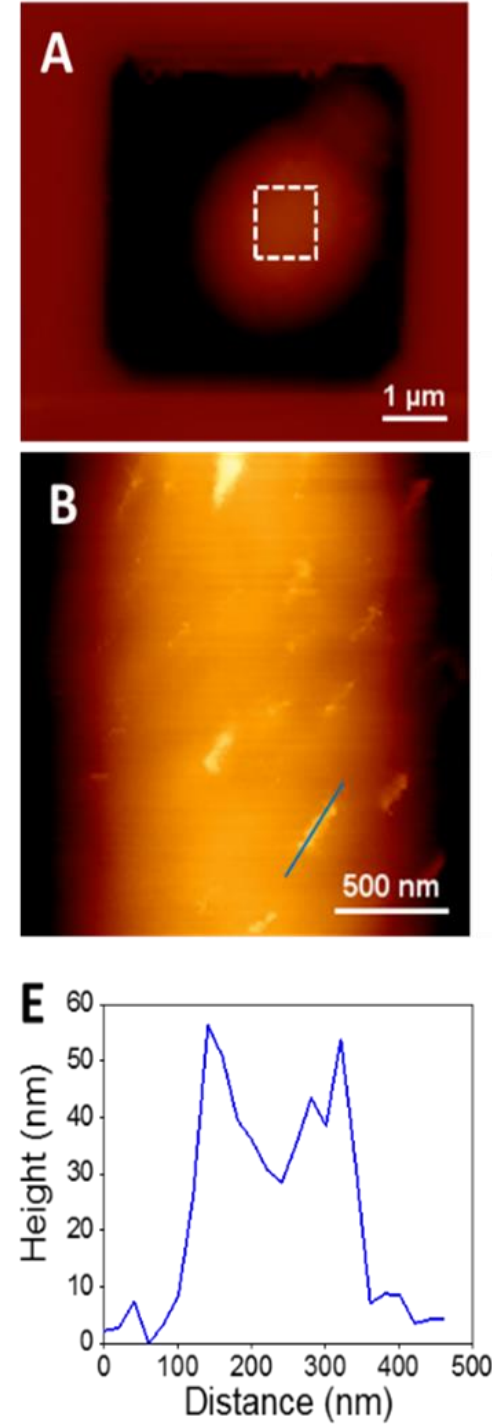

$2 \mu \mathrm{m}$
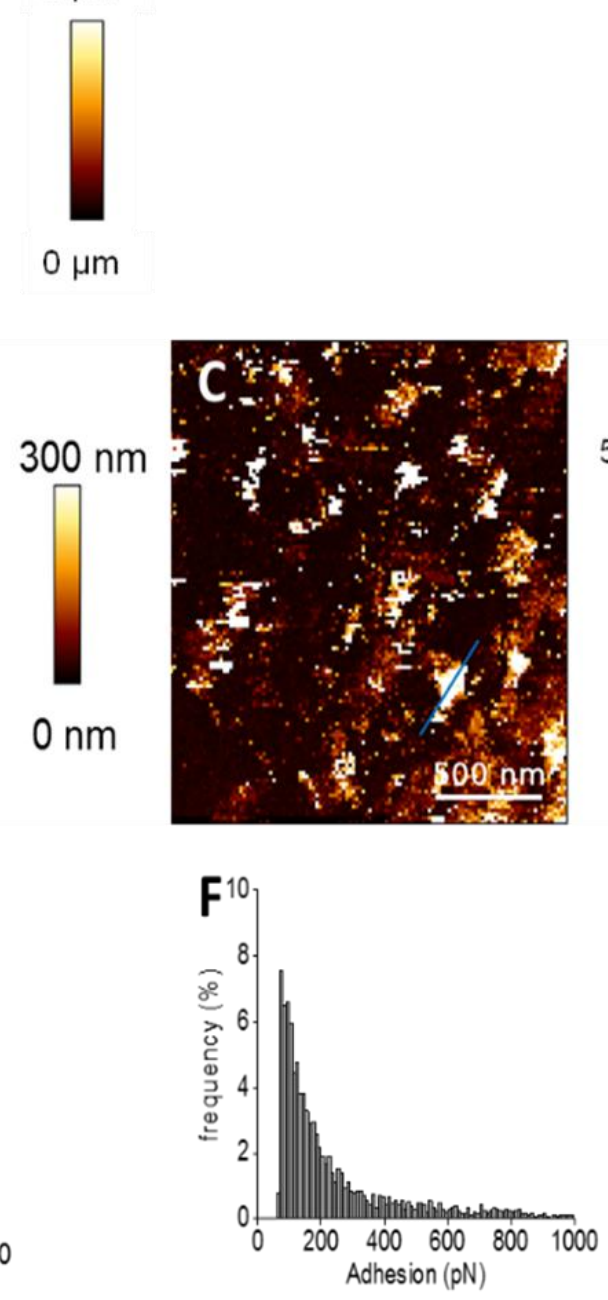

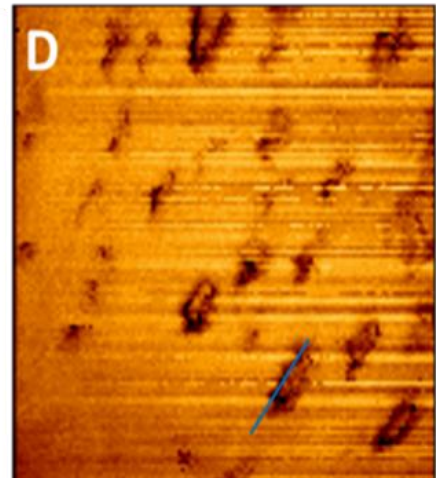

$15 \mathrm{nN} / \mu \mathrm{m}$

$500 \mathrm{pN}$
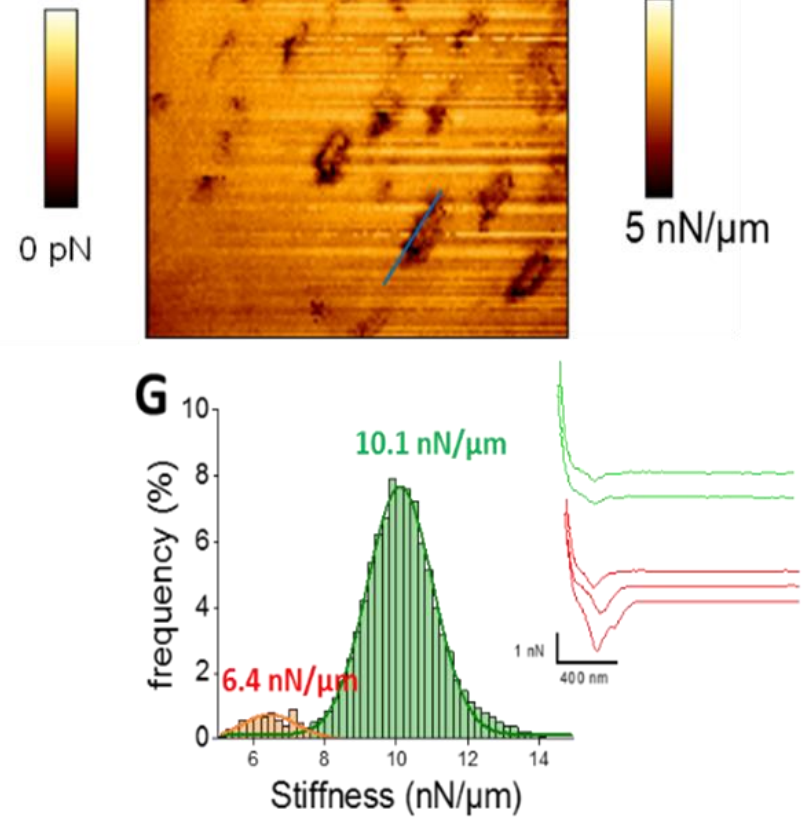

Figure 59: Cell surface analysis of the L69flo11.

$\Delta C$ ter strain using silicon-nitride (Si3N4) AFM cantilevers. AFM height image $(A, B)$, adhesion image (C) and stiffness image (D) of a single yeast cell from L69 flo11- $\Delta$ Cter is shown. In (E) is shown the height of the patches versus their size as indicated by blue line in the adhesion image in (B). in (F) is reported the adhesion forces histogram obtained from 1024 force-distance curve recorded in $\mathrm{QI}^{\mathrm{TM}}$ mode and in (G) the corresponding stiffness, with illustration of a few distance curves on the left of the (G) figure. 


\section{L69flo11- $\Delta R R 1$}
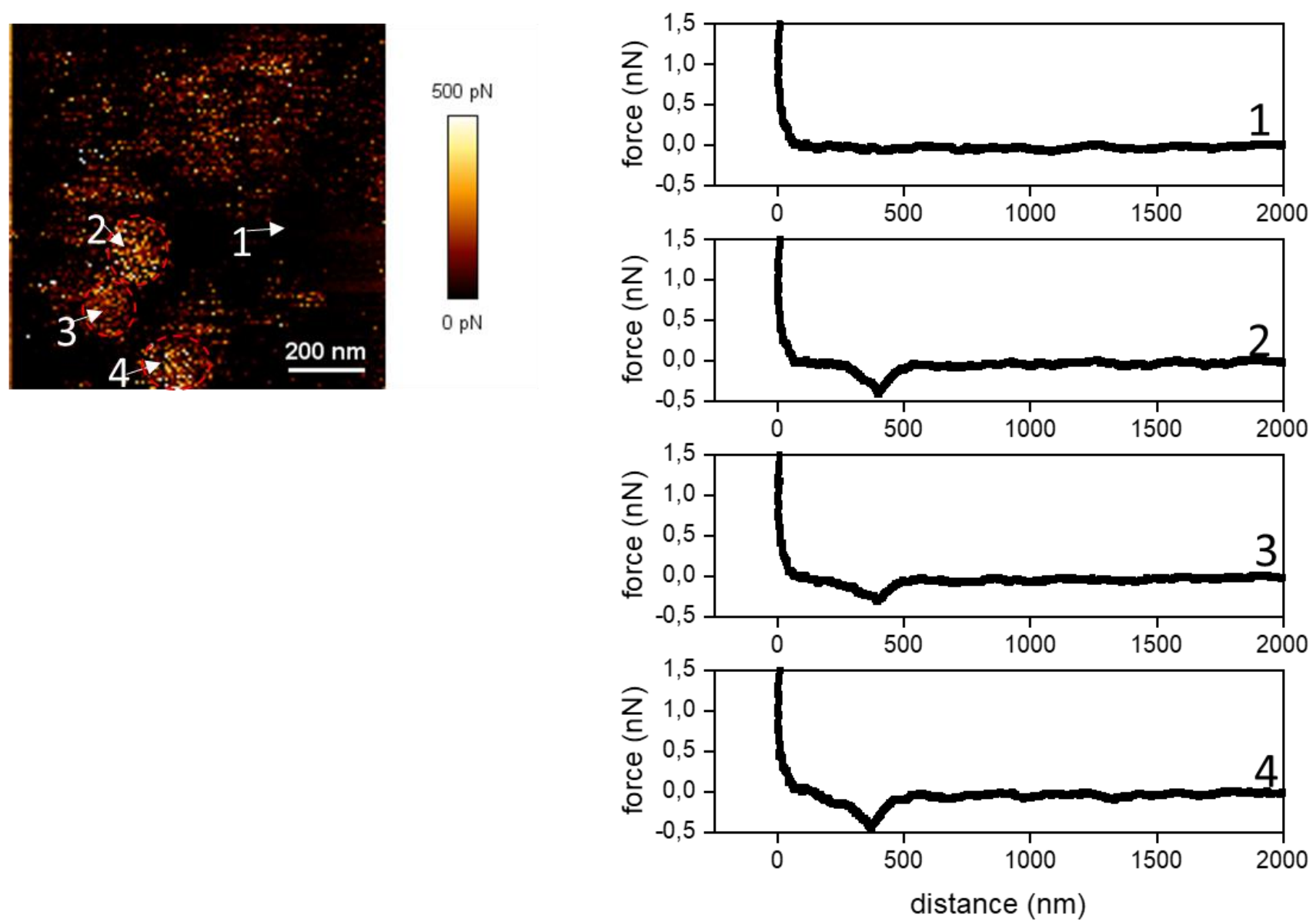

Figure S10: Cell surface analysis of L69flo11- $\triangle R R 1$ using silicon-nitride (Si3N4) AFM cantilevers. Representative force-distance curves (right) at different location on the AFM adhesion image (left) indicated by a number obtained from a single cells of L69flo11- $\triangle R R 1$ embedded in PDMS chamber. 


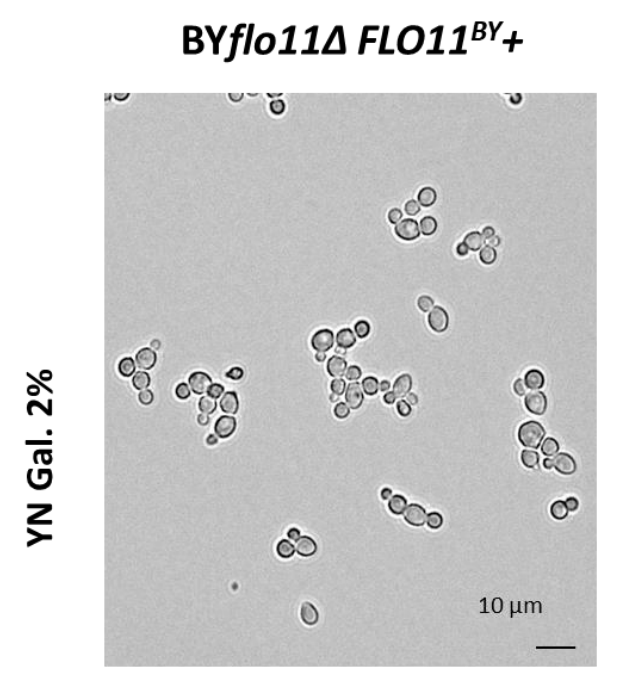

\section{BYflo11 FLO11 $^{169}+$}
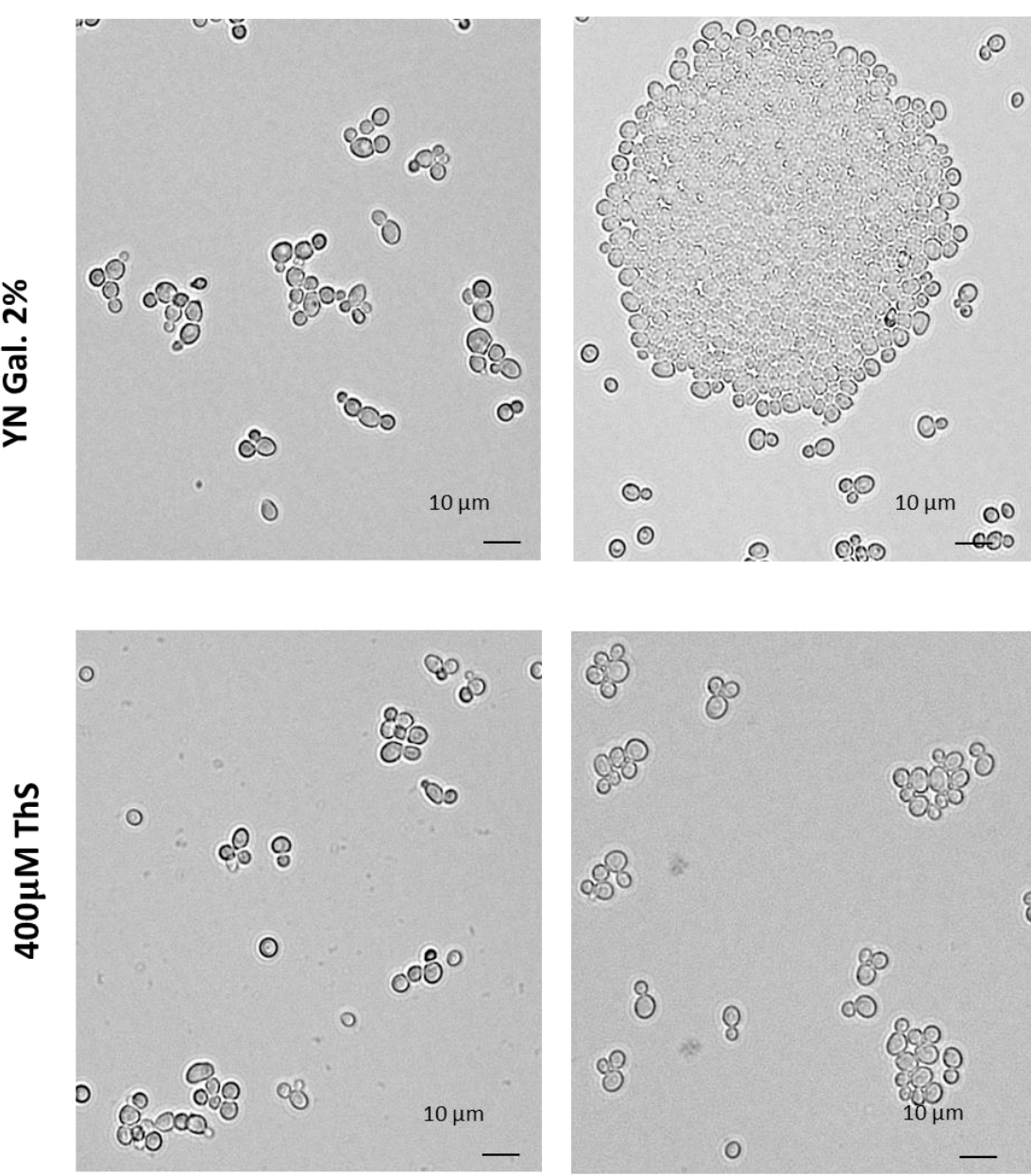

L69

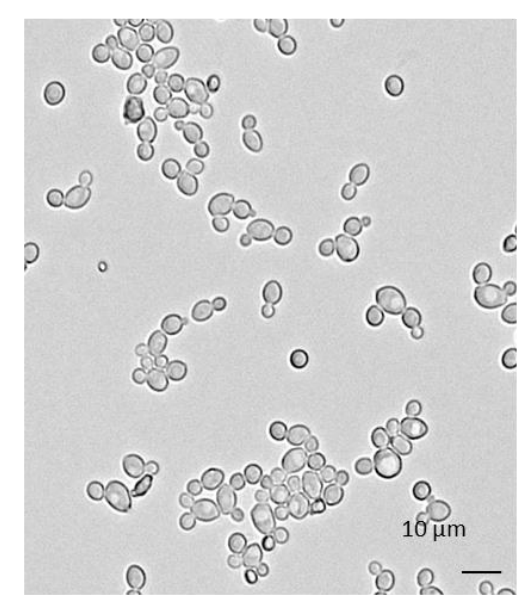

L69flo11- $\triangle R R 2$

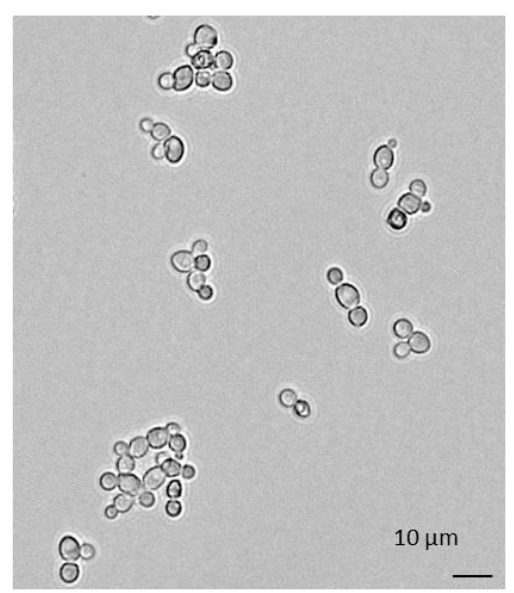

YSWT3 $\alpha$

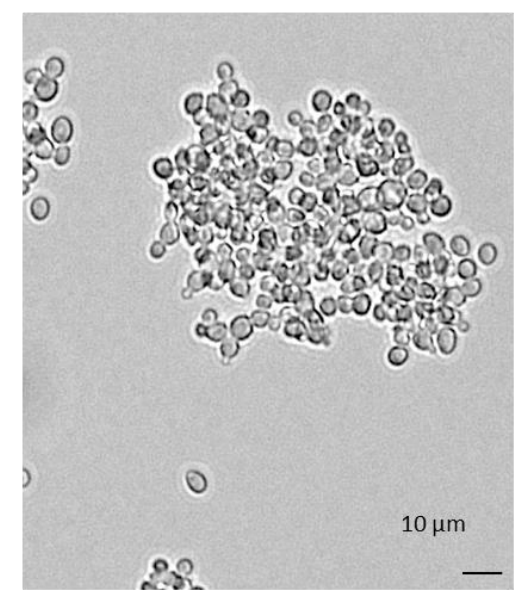

0
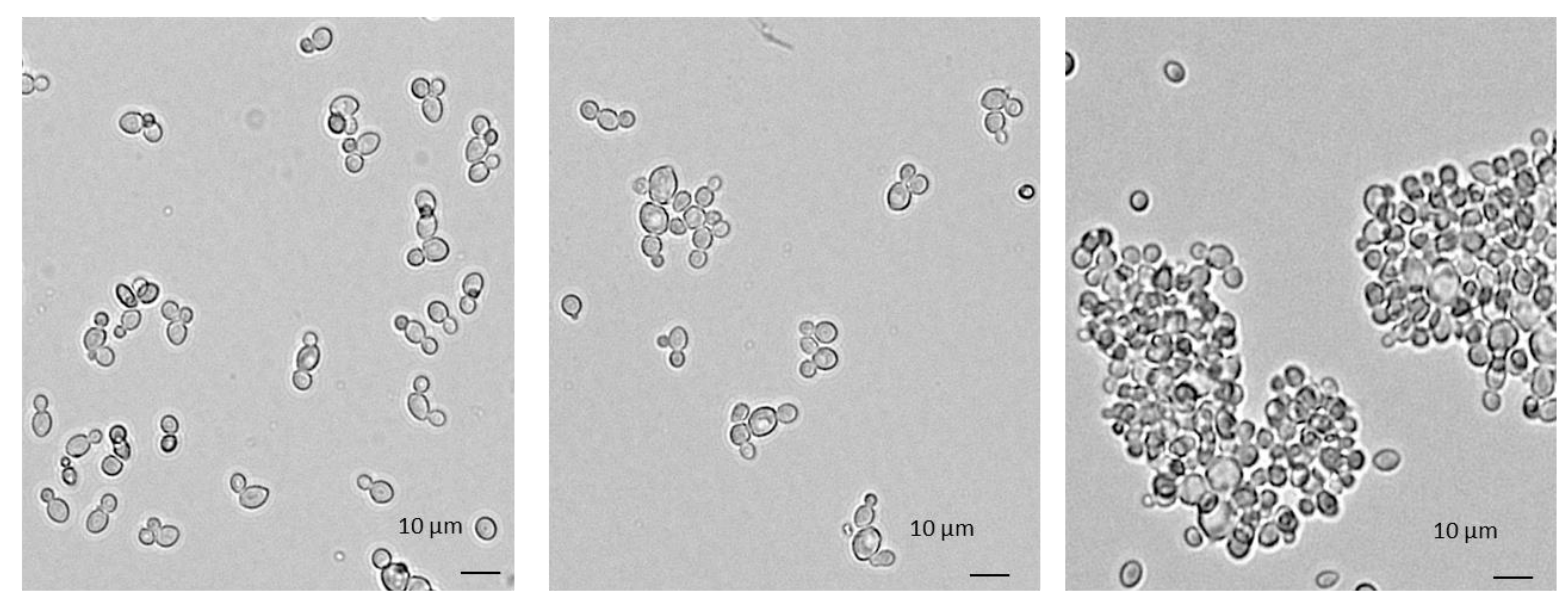

Figure S11: Effect of Thioflavine $S$ on the Flo11p-dependent cell-cell aggregation.

BY4741 transformed with FLO11 ${ }^{B Y}$ or FLO11 L69, L69, L69 expressing FLO11 lacking RR2 (L69flo11- $\triangle R R 2$ ) and $\Sigma 1278 \mathrm{~b}$ were cultivated in YN Gal. Cultures of these strains were harvested in exponential phase (O.D. around 1.0), and treated or not with $0.4 \mathrm{mM}$ thioflavine $\mathrm{S}$ for $1 \mathrm{~h}$ before microscopic observation. 

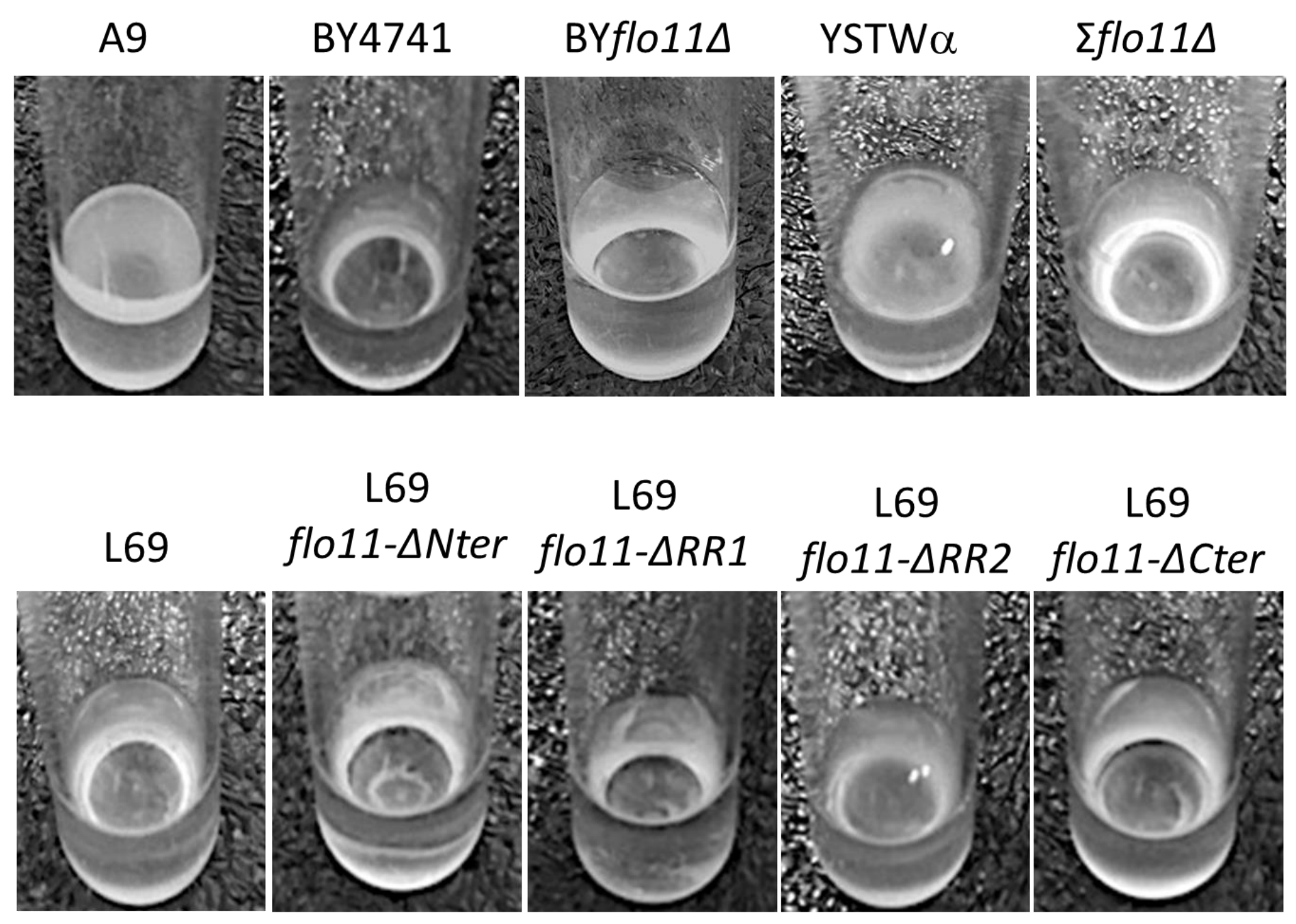

Figure S12: Assay of velum formation. The assay was carried out by static incubation for 7 days of yeast cells in flor medium at $23^{\circ} \mathrm{C}$. 
A

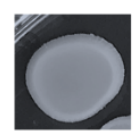

L69

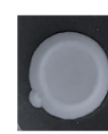

L69flo11 L69flo11

- $\Delta$ Nter $\quad-\Delta R R 1$

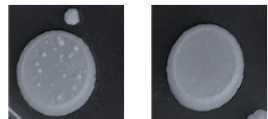

BYflo11A BYflo11A FLO11'BY+FLO11'69+

B

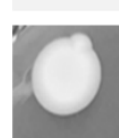

L69

L69flo11

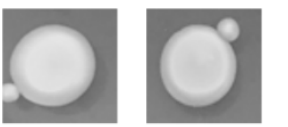

L69flo11 L69flo11

- $\Delta$ Nter $\quad-\triangle R R 1$

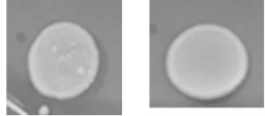

BYflo11A BYflo11A

FLO11 ${ }^{\mathrm{BY}}+\mathrm{FLO11}{ }^{\mathrm{L} 69+}$
YPGal / Before wash

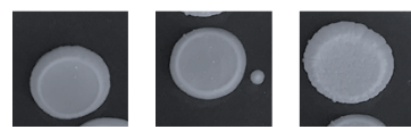

$\Sigma 1278 b$
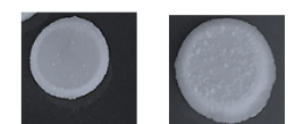

YSWT3 2 Iflo114

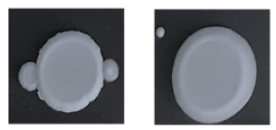

L69flo11 L69flo11

$-\triangle R R 2 \quad-\triangle C$ Cter

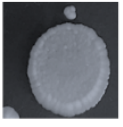

BYflo11A

FLO11 ${ }^{B Y}-[R R 2]^{L 69_{+}}$

YNGIc / Before wash

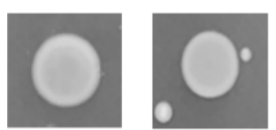

BY4741 BYflo11A

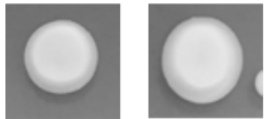

L69flo11 L69flo11

- $\triangle R R 2 \quad-\triangle$ Cter

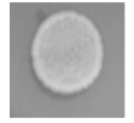

BYflo11

FLO11BY ${ }^{-}[R R 2]^{L 69_{+}}$

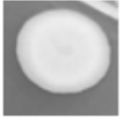

$\Sigma 1278 b$

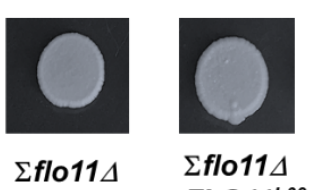

$\mathrm{FLO11}^{\mathrm{BY}}+\mathrm{FLO11}^{\mathrm{L} 69_{+}}$
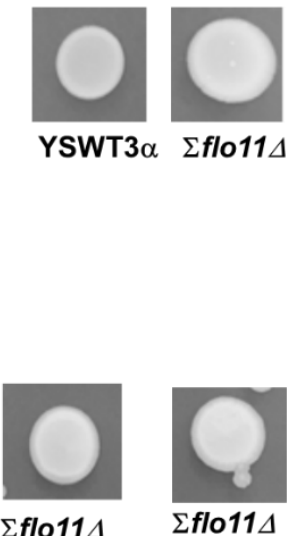

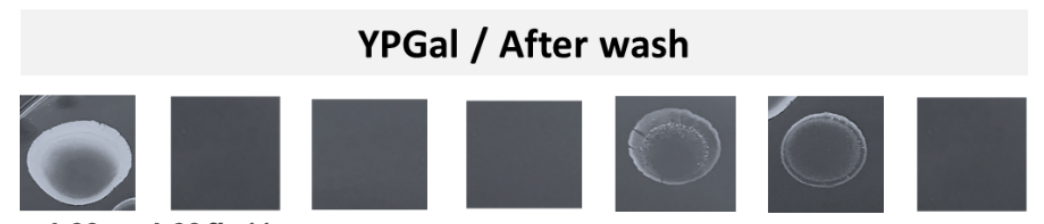

69

L69flo11A BY4741

BYflo11 $\triangle$

$\Sigma 1278 b$

YSWT3 $\alpha$ sflo11

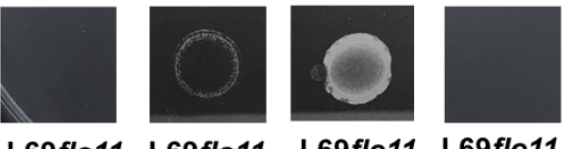

L69flo11 L69flo11 L69flo11 L69flo11

- $\Delta$ Nter $\quad-\Delta R R 1$

$-\triangle R R 2$

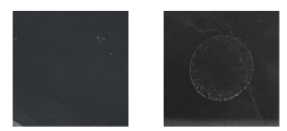

BYflo11 B BYflo114 FLO11 ${ }^{B Y}+F L 011^{L 69+}$
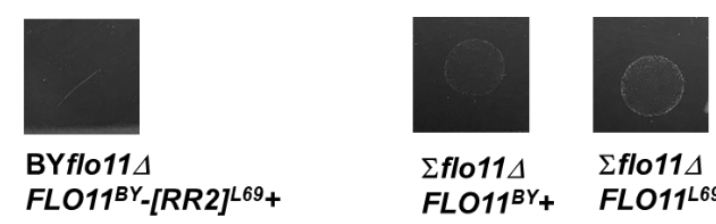

Eflo114 $\Sigma$ flo114 FLO11 ${ }^{\mathrm{BY}}+\mathrm{FLO11}^{\mathrm{L69}+}$

\section{YNGIc / After wash}
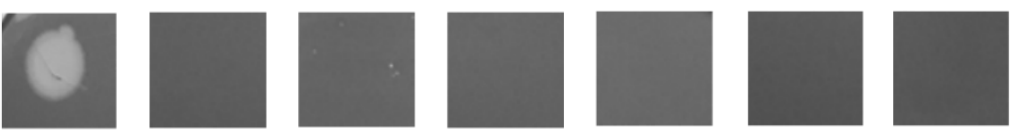

L69

L69flo114

BY4741 BYflo114

$\Sigma 1278 b$

YSWT3 $\alpha \quad \Sigma$ flo114
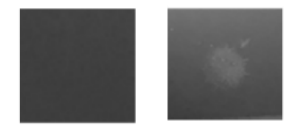

L69flo1

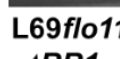

L69flo11
$-\Delta R R 1$

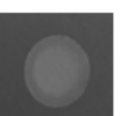

0911

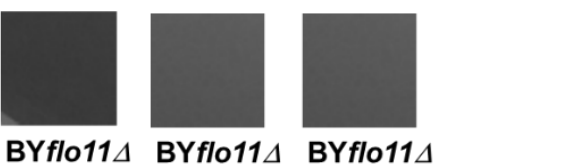

BYflo11 B BYflo11 B BYflo11

FLO11 ${ }^{\mathrm{BY}}+\mathrm{FLO11}{ }^{\mathrm{L} 69+} \mathrm{FLO11}^{\mathrm{BY}}-\left[\mathrm{CRR}^{\mathrm{B}}\right]^{\mathrm{Lg}+}$

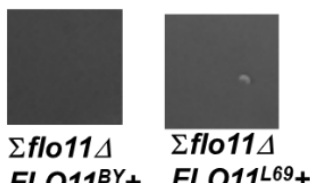

Figure S13: Invasive growth in agar by different yeast strains, and impact of the culture medium and of the domains of FLO11p on this phenotype. Yeast cells were initially cultivated in YNGal complemented with amino acids and uracil, except for BYflo11 11 and $\Sigma$ flo $11 \Delta$ expressing FLO11 ${ }^{B Y}$ or FLO11 ${ }^{169}$ on a pYES2.1 plasmid for which uracil was omitted. Cells were harvested at the entrance in stationary phase and deposited as patches on agar plates which were made with (A) rich galactose medium (YP Gal) or (B) with synthetic glucose medium complemented with amino acids and uracil, except for BYflo11 $\triangle$ and $\Sigma$ flo11 1 expressing $F L O 11^{B Y}$ on $F L O 11^{L 69}$ on a pYES2.1 plasmid for which uracil was omitted. Plates were incubated at $30^{\circ} \mathrm{C}$ for 8 days. They were then photographed before and after washing under a stream of water. 


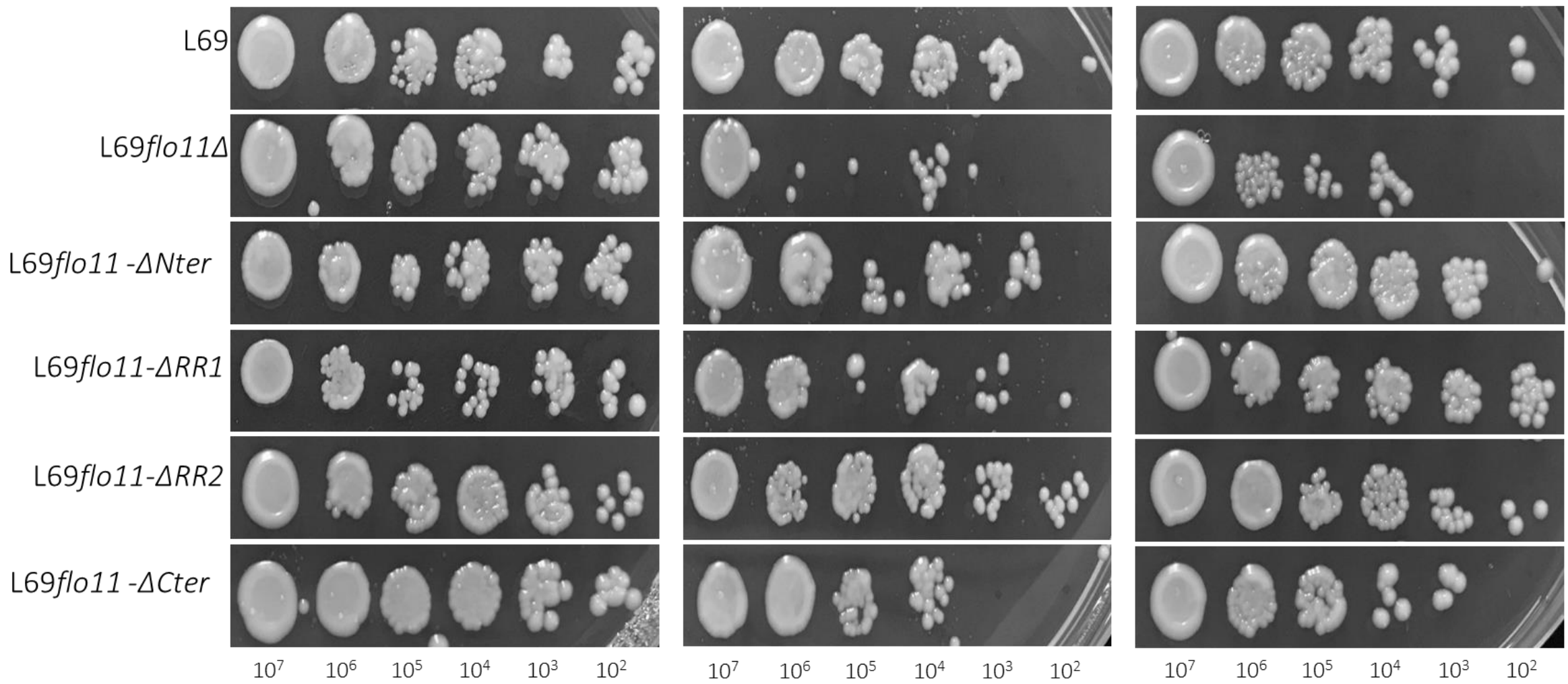

Figure S14: Sensitivity of L69 and L69 mutant strains to Caffeine and Calcofluor White (CFW) drugs. Cells were exponentially grown in YPD and were collected by centrifugation then resuspended in sterilize water at $10^{8} \mathrm{cells}_{\mathrm{mL}}^{-1}$. Series of 10 -fold dilutions were spotted on YPD agar plates in the absence or presence of CFW or Caffeine. Picture was taken after 2 days of growth at $30^{\circ} \mathrm{C}$. 
Table S1. TANGO software analysis of $\beta$-aggregation motifs in Flo1, Flo5, Flo9, Flo10 and Flo11 protein from Saccharomyces cerevisiae S288c strain

\begin{tabular}{|c|c|c|c|c|c|c|c|c|c|c|c|c|c|c|}
\hline \multicolumn{3}{|c|}{ Flo1 } & \multicolumn{3}{|c|}{ Flo5 } & \multicolumn{2}{|c|}{ Flo9 } & \multicolumn{4}{|c|}{ Flo10 } & \multicolumn{3}{|c|}{ Flo11 } \\
\hline $\begin{array}{c}\mathrm{AA} \\
\text { position }\end{array}$ & motif & $\begin{array}{c}\text { Mean } \beta \\
\text { aggregate } \\
(\%)\end{array}$ & $\begin{array}{c}\text { AA } \\
\text { position }\end{array}$ & motif & $\begin{array}{c}\text { Mean } \beta \text { - } \\
\text { aggregate } \\
(\%)\end{array}$ & $\begin{array}{c}\mathrm{AA} \\
\text { position }\end{array}$ & motif & $\begin{array}{c}\text { Mean } \beta \text { - } \\
\text { aggregate } \\
(\%)\end{array}$ & $\begin{array}{c}\mathrm{AA} \\
\text { position }\end{array}$ & motif & $\begin{array}{c}\text { Mean } \beta \text { - } \\
\text { aggregate } \\
(\%)\end{array}$ & $\begin{array}{c}\mathrm{AA} \\
\text { position }\end{array}$ & motif & $\begin{array}{c}\text { Mean } \beta- \\
\text { aggregate } \\
(\%)\end{array}$ \\
\hline $7-21$ & $\begin{array}{c}\text { YMFLAVFTLLAL } \\
\text { TSV }\end{array}$ & 82.2 & $9-22$ & IFLVILAFLALINVA & 82 & $6-22$ & $\begin{array}{l}\text { YCLLLAIVTLLG } \\
\text { LTNVV }\end{array}$ & 77.55 & $7-23$ & $\begin{array}{c}\text { YIFLTGLFLLSVANV } \\
\text { AL }\end{array}$ & 81.7 & $5-16$ & FLLAYLVLSLLF & 96 \\
\hline $308-312$ & TVIVI & 87.9 & $207-215$ & TVYMYAGYY & 30.5 & $118-122$ & IIAYW & 72 & $1028-1035$ & VVTVYSTW & 48.5 & $1033-1042$ & VTTVVSTTVV & 75.8 \\
\hline $353-357$ & TIIVI & 87.3 & $308-312$ & TVIVI & 73.2 & $207-215$ & TVYMYAGFY & 50.9 & $1115-1119$ & VLISV & 47 & $1056-1061$ & ITTTFV & 56 \\
\hline $398-402$ & TIIVI & 87.3 & $353-357$ & TVIVI & 87.8 & $308-312$ & TVIVI & 87.9 & $1157-1168$ & ISIFIASLLLAI & 89.8 & $1133-1144$ & TLVTTAVTTTVV & 84.8 \\
\hline $443-447$ & TIIVI & 87.3 & $398-402$ & TVIVI & 87.8 & $353-357$ & TIIVI & 87.3 & & & & $1356-1362$ & FMWLLLA & 85.3 \\
\hline $488-492$ & TIIVI & 87.3 & $443-447$ & TVIVI & 87.8 & $398-402$ & TIIVI & 87.3 & & & & & & \\
\hline $533-537$ & TIIVI & 87.2 & $488-492$ & TVIVI & 87.8 & $443-447$ & TIIVI & 87.3 & & & & & & \\
\hline $578-562$ & TIIVI & 87.2 & $533-537$ & TVIVI & 87.9 & $498-492$ & TIIVI & 87.3 & & & & & & \\
\hline $623-627$ & TIIVI & 87.2 & $578-582$ & TVIVI & 87.9 & $533-537$ & TIIVI & 87.2 & & & & & & \\
\hline $667-672$ & TIIVI & 87.2 & $623-627$ & TVIVI & 87.9 & $578-582$ & TIIVI & 87.2 & & & & & & \\
\hline $713-717$ & TIIVI & 87.2 & $783-788$ & TLVTVT & 31.7 & $623-627$ & TIIVI & 87.2 & & & & & & \\
\hline $758-762$ & TVIVI & 87.8 & $802-811$ & AIVSTATVTV & 45.2 & $668-672$ & TIIVI & 87.2 & & & & & & \\
\hline $803-807$ & TVIVI & 87.8 & $855-859$ & TVVTI & 37.2 & $713-717$ & TIIVI & 87.2 & & & & & & \\
\hline $848-852$ & TVIVI & 87.8 & $906-911$ & TLVTVT & 36.1 & $758-762$ & TVIVI & 87.8 & & & & & & \\
\hline 893-897 & TVIVI & 87.8 & $1063-1074$ & LSVFIASLLLAI & 87 & $803-807$ & TVIVI & 87.8 & & & & & & \\
\hline $938-942$ & TVIVI & 87.8 & & & & $848-852$ & TVIIV & $87 ., 7$ & & & & & & \\
\hline $983-987$ & TVIVI & 87.8 & & & & $1021-1026$ & TLVTVT & 31.9 & & & & & & \\
\hline 1028-1032 & TVIVI & 87.9 & & & & $1040-1049$ & AIVSTATVTV & 42.6 & & & & & & \\
\hline 1073-1077 & TVIVV & 88.5 & & & & 1093-1097 & TVVTI & 37.4 & & & & & & \\
\hline $1234-1239$ & TLVTVT & 31.7 & & & & $1144-1149$ & TLVTVT & 37.7 & & & & & & \\
\hline $1254-1262$ & IVSTATVTV & 45.9 & & & & $1175-1182$ & VVTVYSTW & 82.6 & & & & & & \\
\hline $1299-1303$ & TVVTI & 37.2 & & & & $1310-1321$ & LSVFIASLLLAI & 87 & & & & & & \\
\hline $\begin{array}{l}1350-1355 \\
1525-1536\end{array}$ & $\begin{array}{c}\text { TLVTVT } \\
\text { LSVFIASLLLAI }\end{array}$ & $\begin{array}{l}36 \\
87\end{array}$ & & & & & & & & & & & & \\
\hline
\end{tabular}


Table S2. Search for intragenic repeats using EMBOSS ETANDEM software

\begin{tabular}{|c|c|c|c|c|c|c|c|c|}
\hline $\begin{array}{l}\text { Gene } \\
\text { name }\end{array}$ & $\begin{array}{l}\text { ORF } \\
\text { (bp) }\end{array}$ & $\begin{array}{c}\text { Repetition } \\
\text { length } \\
\text { (TR) }\end{array}$ & Score & count & $\begin{array}{c}\text { Repetition } \\
\text { start } \\
\text { (nt. seq.) }\end{array}$ & $\begin{array}{l}\text { Repetition } \\
\text { stop } \\
\text { (nt. seq.) }\end{array}$ & $\begin{array}{c}\text { Repetition } \\
\text { conservation } \\
(\%) \\
\end{array}$ & Repeated sequence (consensus) \\
\hline \multirow{3}{*}{$F L O 11^{169}$} & \multirow{3}{*}{5166} & 63 & 323 & 30 & 658 & 2547 & 60.2 & $\begin{array}{l}\text { acttcatctaccgctactactgcaaccacttctactactgcaaccact } \\
\text { tctactactgcaaca }\end{array}$ \\
\hline & & 45 & 95 & 4 & 2646 & 2825 & 88.9 & accagctccaactccatccagctctactactgaaagctcttctgc \\
\hline & & 45 & 71 & 4 & 2958 & 3137 & 82.2 & atccagctctaccactgaaagctcttctgctccagtatcaacccc \\
\hline \multirow{3}{*}{$F L O 11^{\mathrm{BY}}$} & \multirow{3}{*}{4104} & 63 & 304 & 24 & 688 & 2388 & 60.8 & $\begin{array}{l}\text { tctactacagcaaccacttcaaccaccgcaactactgcaaccactt } \\
\text { ctactactgaaaccact }\end{array}$ \\
\hline & & 33 & 55 & 8 & 2832 & 3095 & 66.7 & ctctgcatgaacaaccactaccactacaactac \\
\hline & & 45 & 48 & 3 & 2429 & 2563 & 84.4 & caaccccatcaagctctagcactgaaagctcttctgctccagtat \\
\hline \multirow{3}{*}{$F L O 11^{\Sigma}$} & \multirow{3}{*}{3633} & 45 & 94 & 5 & 1923 & 2147 & 80.9 & cactgaaagctcttctgctccagtaccaactccatccagctctag \\
\hline & & 45 & 46 & 3 & 2158 & 2292 & 83.7 & ccagtaccaactccatccagctctagcactgaaagctcctctgct \\
\hline & & 45 & 29 & 2 & 335 & 424 & 91.1 & gttgcgacgaaaatacctatttgattgacaacccaactgatttca \\
\hline$F L O 11^{133 d}$ & 4890 & 81 & 1708 & 49 & 827 & 4795 & 72.5 & $\begin{array}{l}\text { cttcttctgctccagttacttcttctactactgaatcttcttctgctcca } \\
\text { gctcctactccttcttcttctactactgaat }\end{array}$ \\
\hline
\end{tabular}

$F L O 11^{69}=F L O 11$ gene from $L 69$ strain; $F L O 11^{B Y}=$ FLO11 gene from BY4741 strain; FLO11 ${ }^{\Sigma}=$ FLO11 gene from $\Sigma 1278 \mathrm{~b} ; F L O 11^{133 d}=$ FLO11 gene from $133 \mathrm{~d}$ flor strain 
Table S3. Search for $\beta$-aggregation prone sequence in the different Flo11 proteins using TANGO software (http://tango.crg.es/)

\begin{tabular}{|c|c|c|c|}
\hline $\begin{array}{c}\text { Protein } \\
\text { (strain origin) }\end{array}$ & Amino-acid position & Motif & $\begin{array}{c}\text { Mean } \beta \text { aggregation } \\
(\%)\end{array}$ \\
\hline \multirow{9}{*}{$\begin{array}{l}\text { Flo11p }^{69} \\
\text { (L69) }\end{array}$} & $5 . .16$ & FLLAYLVLSLLF & 96 \\
\hline & $1178 \ldots 1187$ & VTTVVSTTVV & 75.7 \\
\hline & $1201 \ldots 1206$ & ITTTFV & 55.8 \\
\hline & $1285 \ldots 1294$ & VTTVVSTTVV & 75.7 \\
\hline & $1308 \ldots . .1313$ & ITTTFV & 55.8 \\
\hline & $1392 \ldots 1401$ & VTTVVSTTVV & 75.7 \\
\hline & $1415 \ldots 1420$ & ITTTFV & 55.8 \\
\hline & $1494 \ldots 1503$ & VTTAVTTTVV & 59.4 \\
\hline & $1710 \ldots 1716$ & FMWLLLA & 85.3 \\
\hline \multirow{5}{*}{$\begin{array}{l}\text { Flo11p } \\
\text { (BY4741) }\end{array}$} & $5 \ldots 16$ & FLLAYLVLSLLF & 96 \\
\hline & $1033 \ldots 1042$ & VTTVVSTTVV & 75.8 \\
\hline & $1056 \ldots 1061$ & ITTTFV & 56 \\
\hline & $1133 \ldots 1144$ & TLVTTAVTTTVV & 84.8 \\
\hline & $1356 \ldots 1362$ & FMWLLLA & 85.3 \\
\hline \multirow{5}{*}{$\begin{array}{l}\text { Flo11p } \\
(\Sigma 1278 b)\end{array}$} & $5 \ldots 20$ & FAYLVLSLLFYSAL & 83 \\
\hline & $881 \ldots 890$ & VTTVVSTTVV & 75.6 \\
\hline & $904 \ldots 909$ & ITTTFV & 55.4 \\
\hline & $983 \ldots 992$ & VTTAVTTTVV & 59 \\
\hline & $1199 \ldots 1205$ & FMWLLLA & 85.3 \\
\hline \multirow{5}{*}{$\begin{array}{l}\text { Flo11p }^{133 d} \\
(133 d)\end{array}$} & $5 \ldots 16$ & FLLAYLVLSLLF & 96 \\
\hline & $1301 \ldots 1311$ & VTTVVSTTVVT & 70.9 \\
\hline & $1324 \ldots 1330$ & ITTTFVT & 50.7 \\
\hline & 1403..1412 & VTTAVTTTVV & 59.1 \\
\hline & $1619 \ldots 1625$ & FMWLLLA & 85.3 \\
\hline
\end{tabular}

*Beta-aggregation prone sequences $>30 \%$ were searched using TANGO software (at http://tango.crg.es/) with default setting of $\mathrm{pH}$, ionic strength and temperature. Amyloid-core sequences are highlighted in yellow. 
Table S4. Yeast strains used or constructed in this study.

\begin{tabular}{|c|c|c|}
\hline Strain & Genotype/Remarks & Source \\
\hline L69 & Saccharomyces cerevisiae prototrophic diploid. & Lallemand Inc. \\
\hline L69flo11 & L69 flo11::KanMX4. & This study \\
\hline L69flo11-Nter & L69 diploid strain expressing FLO11 deleted of its $\mathrm{N}$-terminus domain. & This study \\
\hline L69flo11 $-R R 1$ & L69 diploid strain expressing FLO11 deleted of the intragenic RR1. & This study \\
\hline L69flo11 $-R R 2$ & L69 diploid strain expressing FLO11 deleted of the RR2 intragenic domain & This study \\
\hline L69flo11 11 -Cter & L69 diploid strain expressing FLO11 deleted of its C-terminal domain & This study \\
\hline BY4741 & MATa his3 $\Delta 1$ leu2 $2 \Delta 0$ met $15 \Delta 0$ ura3 $\Delta 0$. & Euroscarf \\
\hline BYflo11 $1 \Delta$ & BY4741 flo11::KanMX4. & YKO Open biosystem \\
\hline$\Sigma 1278 b$ & Prototophic diploid strain & Lab source \\
\hline YSWT3 $\alpha$ & MAT $\alpha$ can1 $\Delta::$ Ste2pr-spHIS5 lyp1 $\Delta::$ Ste3pr-LEU2 his3::hisG leu2 $\Delta 0$ ura3 $\Delta 0$. Derived from $\Sigma 1278 b$ & C.Boone (Univ. Toronto) \\
\hline$\Sigma$ flo11 & YSWT3 $\alpha$ flo11 ::kanMX4 & C.Boone (Univ. Toronto) \\
\hline BYflo11 $F L O 11^{\mathrm{BY}+}$ & BY4741 flo11::KanMX4 transformed with pYES FLO11 ${ }^{\mathrm{BY}}$. & This study \\
\hline BYflo11 $F L O 11^{169+}$ & BY4741 flo11::KanMX4 transformed with pYES-FLO11 ${ }^{169}$ & This study \\
\hline BYflo11 $\triangle F L O 11^{\mathrm{BY}}[\mathrm{RR} 2]^{\mathrm{Lg}+}$ & BY4741 flo11::KanMX4 haploid strain transformed with pYES-FLO11[RR2] $]^{169}$ & This study \\
\hline$\Sigma$ flo11 $F L O 11^{\mathrm{BY}+}$ & YSWT3 $\alpha$ flo11 ::kanMX4 strain transformed with pYES-FLO11 ${ }^{\mathrm{BY}}$ & This study \\
\hline$\Sigma$ flo11 $F_{L O 11^{169+}}$ & YSWT3 $\alpha$ flo11 ::kanMX4 strain transformed with pYES-FLO11 ${ }^{169} \mathrm{p}$ & This study \\
\hline BY4741+pFLO8 & BY4741 transformed with pGP564_FLO8 vector. & This study \\
\hline A9 & MATa/MATHO/HO & $\begin{array}{l}\text { M. Budroni (Univ di } \\
\text { Sassari, Italy) }\end{array}$ \\
\hline
\end{tabular}


Table S5: Plasmids constructed in this work

\begin{tabular}{|c|c|c|}
\hline Plasmids & description & Source \\
\hline pYES2.1 TOPO TA & $\begin{array}{l}\text { E.coli /yeast shuttle vector with His Tag (6x), V5 Epitope Tag, Amp }{ }^{R} \text {, } \\
\text { GAL1 promotor, URA3 marker. }\end{array}$ & $\begin{array}{l}\text { Life } \\
\text { Technologies }\end{array}$ \\
\hline pYES-FLO11 169 & Cloning of $F L O 11^{169}$ into pYES2.1 TOPO vector. & This study \\
\hline pYES-FLO11 ${ }^{\text {BY }}$ & Cloning of $F L O 11^{\mathrm{BY}}$ into PYES2.1 TOPO vector. & This study \\
\hline pYES-FLO11 ${ }^{B Y}-[R R 2]^{L 69}$ & Cloning of $F L O 11^{B Y}[R R 2]^{L 69}$ chimeric gene in pYES2.1 & This study \\
\hline pGP564_FLO8 & pGP564 vector carrying GLE2, YER107W-A, FLO8, KAP123 and SWI4 & Open Biosystem \\
\hline
\end{tabular}


Table S6. Oligonucleotides used in this study
Oligonucleotides
Nucleic acid sequence (5'-3')
/gRNA sequence

\section{Construction of Flo11 variant in L69 strain}

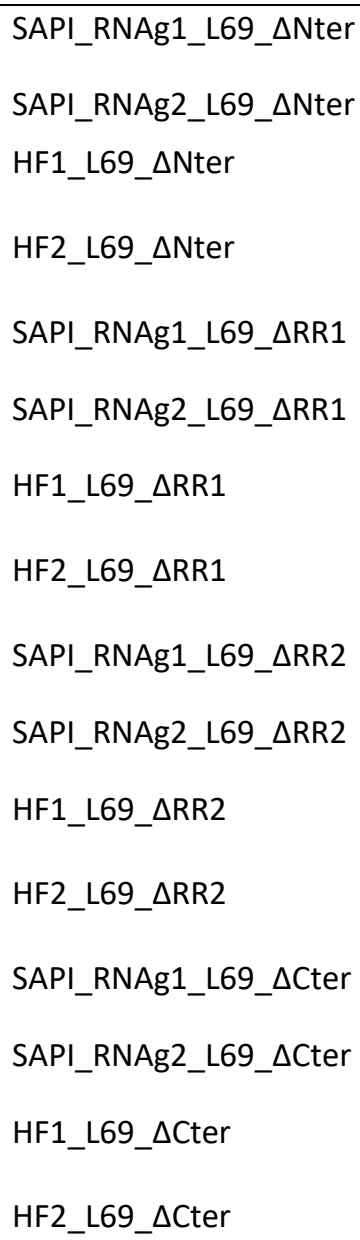

atccaaatcactattcatgtcaa

aacttgacatgaatagtgatttg

aatgtccgtgttcaaattaaataaaaatttagggcagttttatttaccttaacaaatatgacatctagcacttccgagt catctaccactacatcaactaccacttcagaatcatctacat atgtagatgattctgaagtggtagttgatgtagtggtagatgactcggaagtgctagatgtcatatttgttaaggtaa ataaaactgccctaaatttttatttaatttgaacacggacatt atctcagtggtggagctggatac

aacgtatccagctccaccactga

catctagcacttccgagtcatctaccactacatcaactaccacttcagaatcatctacatcaactccatccagctctac tactgaaagctcttctgctccagtaccaaccccatcaagc gcttgatggggttggtactggagcagaagagctttcagtagtagagctggatggagttgatgtagatgattctgaag tggtagttgatgtagtggtagatgactcggaagtgctagatg atctctactagtacaaaaccagg

aacctggttttgtactagtaga

cttcacctaccacacctgtaactacagttgtctcaaccaccgtcgttactactgagtattactctgccggtgaaactac ctctggatgctctccaaagactgtcacaaccactgttcctt

aaggaacagtggttgtgacagtctttggagagcatccagaggtagtttcaccggcagagtaatactcagtagtaacg acggtggttgagacaactgtagttacaggtgtggtaggtgaag atctccgctggtaagacgacaac

aacgttgtcgtcttaccagcgga

tgagtattctactagtacaaaaccaggtggtgaaattacaactacatttgtcaccaaaaataaatacaattccaaca tgttcgtttcttcattacgatgttttcattcttaaattaagat atcttaatttaagaatgaaaacatcgtaatgaagaaacgaacatgttggaattgtatttatttttggtgacaaatgta gttgtaatttcaccacctggttttgtactagtagaatactca

\section{Oligos for the construction of plasmids}

\begin{tabular}{ll}
\hline FLO11_TOPO_f & actatgcaaagaccatttctactcg \\
FLO11_TOPO_r & gaatacaactggaagagcgagtagc \\
FLO11_BY_2964_r & gcaaactgtagtagtaatagtggttgttga \\
R269 inFLO11BY_f & tcaacaaccactattactactacagtttgc
\end{tabular}

\section{Oligos used for the RT-qPCR}

\begin{tabular}{ll}
\hline FLO11_Nt_f & acccaactgatttcacagccac \\
FLO11_Nt_r & agcttgcatattgagcggcac \\
FLO11_Ct_f & gcaagcgcaggcgaaaacac \\
FLO11_Ct_r & gattgaaatagggttggttgctgtg \\
TAF10_f & atattccaggatcaggtcttccgtagc
\end{tabular}


bioRxiv preprint doi: https://doi.org/10.1101/2021.04.01.438097; this version posted April 5, 2021. The copyright holder for this preprint (which was not certified by peer review) is the author/funder, who has granted bioRxiv a license to display the preprint in perpetuity. It is made available under aCC-BY 4.0 International license.

TAF10_r

UBC6_f

UBC6_r gtagtcttctcattctgttgatgttgttgttg

gatacttggaatcctggctggtctgtctc

aaagggtcttctgtttcatcacctgtatttgc 